TRANSACTIONS OF THE

AMERICAN MATHEMATICAL SOCIETY

Volume 357, Number 9, Pages 3683-3723

S 0002-9947(05)03645-7

Article electronically published on February 4, 2005

\title{
ASYMPTOTIC PROPERTIES OF CONVOLUTION OPERATORS AND LIMITS OF TRIANGULAR ARRAYS ON LOCALLY COMPACT GROUPS
}

\author{
YVES GUIVARC'H AND RIDDHI SHAH
}

\begin{abstract}
We consider a locally compact group $G$ and a limiting measure $\mu$ of a commutative infinitesimal triangular system (c.i.t.s.) $\Delta$ of probability measures on $G$. We show, under some restrictions on $G, \mu$ or $\Delta$, that $\mu$ belongs to a continuous one-parameter convolution semigroup. In particular, this result is valid for symmetric c.i.t.s. $\Delta$ on any locally compact group $G$. It is also valid for a limiting measure $\mu$ which has 'full' support on a Zariski connected $\mathbb{F}$-algebraic group $G$, where $\mathbb{F}$ is a local field, and any one of the following conditions is satisfied: (1) $G$ is a compact extension of a closed solvable normal subgroup, in particular, $G$ is amenable, (2) $\mu$ has finite one-moment or (3) $\mu$ has density and in case the characteristic of $\mathbb{F}$ is positive, the radical of $G$ is $\mathbb{F}$-defined. We also discuss the spectral radius of the convolution operator of a probability measure on a locally compact group $G$, we show that it is always positive for any probability measure on $G$, and it is also multiplicative in case of symmetric commuting measures.
\end{abstract}

\section{INTRODUCTION}

In probability theory, one-parameter convolution semigroups on $\mathbb{R}^{n}$ play an important role and often arise as limits of partial products of infinitesimal triangular systems (arrays), and it is a celebrated theorem that in Euclidean space such a limit $\mu$ always belongs to a one-parameter convolution semigroup. In this paper, we show under mild conditions that this property remains valid in the general setting of a locally compact group $G$, instead of $\mathbb{R}^{n}$, provided the triangular system is commutative and in particular if $G$ is of "Lie type" over one of the three classes of local fields. Our work corresponds to a part of the solution of the so-called central limit problem for the convolution structure on $G$ (see the introduction in $[\mathrm{H}])$. The aspects of this problem connected with the normalisation of iterates of a given probability measure $\mu$ are not considered in this paper. We refer to [G4] for a survey on these important aspects.

Let $G$ be a locally compact (Hausdorff, second countable) group with identity $e$ and let $M^{1}(G)$ be the topological convolution semigroup of probability measures on $G$ endowed with weak topology. For $x \in G$, let $\delta_{x}$ denote the Dirac measure supported on $x$. An element $\mu \in M^{1}(G)$ is said to be embeddable if there exists

Received by the editors July 30, 2003 and, in revised form, February 12, 2004.

2000 Mathematics Subject Classification. Primary 60B15, 60F05, 60G50; Secondary 43A05, 22D25, 22D40.

Key words and phrases. Infinitesimal triangular systems of measures, embeddable measures, spectrum of convolution operators, Lyapunov exponents, algebraic groups.

(C)2005 American Mathematical Society 
a continuous one-parameter convolution semigroup $\left\{\mu_{t}\right\}_{t \geq 0}$ in $M^{1}(G)$ such that $\mu_{1}=\mu$. In this case the probability measure $\mu_{0}=\lim _{t \rightarrow 0} \mu_{t}$ is an idempotent, hence is the normalised Haar measure $\omega_{K}$ of a compact subgroup $K$ (cf. $[\mathrm{P}$, p. 62). A sequence of finite ordered sets of $M^{1}(G), \Delta=\left\{\mu_{i j} \in M^{1}(G) \mid i \in \mathbb{N}, 1 \leq\right.$ $\left.j \leq n_{i}, \lim _{i \rightarrow \infty} n_{i}=\infty\right\}$ is said to be a triangular system in $M^{1}(G)$; we will sometimes write $\Delta=\left(\mu_{i j}\right)_{i \in \mathbb{N}, j=1}^{n_{i}} . \Delta$ is said to be commutative if for every fixed $i$, $\mu_{i j}$ commutes with $\mu_{i j^{\prime}}$ for all $j, j^{\prime}$; it is said to be $\omega_{K}$-infinitesimal if as $i \rightarrow \infty$, $\lim _{i \rightarrow \infty} \mu_{i j}=\omega_{K}$ uniformly in $j$. We say that $\Delta$ converges to $\mu$ if $\lim _{i \rightarrow \infty} \mu_{i 1} *$ $\cdots * \mu_{i n_{i}}=\mu$, where '*' denotes the convolution of measures. For brevity we say that $\Delta$ is a c.i.t.s. if $\Delta$ is a commutative infinitesimal triangular system. Clearly, if $\Sigma=\left\{\mu_{t}\right\}_{t \geq 0} \subset M^{1}(G)$ is a continuous one-parameter semigroup with $\mu_{0}=\omega_{K}$, then $\mu_{1}$ is the limit of a c.i.t.s. This is valid more generally if $\mu$ belongs to a semigroup of the form $C \Sigma=\left\{\delta_{x} * \mu_{t} \mid x \in C, t \geq 0\right\} \subset M^{1}(G)$, where $\Sigma$ is as above and $C$ is a compact connected abelian subgroup whose elements commute with those of $\Sigma$. Let $\Sigma=\left\{\mu_{t}\right\}_{t \geq 0}$ be a continuous one-parameter semigroup with $\mu_{0}=\omega_{H}$ for some compact subgroup $H \subset G$, and let $C$ be a compact subgroup whose elements commute with those of $\Sigma$, contains $H$ as a normal subgroup and $C / H$ is connected and abelian. We say that $\mu$ is quasi-embeddable in $\Sigma$ relative to $C$ if $\mu \in C \Sigma \subset \omega_{H} M^{1}(G) \omega_{H}=M_{H}^{1}(G)$.

It is easy to see that quasi-embeddability of $\mu$ implies that $\mu$ is the limit of a c.i.t.s. in $M_{H}^{1}(G)$. Also, it implies the infinite divisibility of $\mu$, i.e. the existence of an $n$th root for every $n \in \mathbb{N}$ and embeddability of $x \mu=\delta_{x} * \mu$ for some $x \in C$. We conjecture that for a large class of locally compact groups including all the connected Lie groups, if $\mu$ is the limit of a c.i.t.s. and if $Z_{\mu}^{0} / Z^{0}$ is compact (see notations below), then it is quasi-embeddable and we prove this property under some mild conditions. In order to simplify this exposition, we will only consider c.i.t.s. such that $\omega_{K}=\delta_{e}$. We may note here that all the relevant results are valid for a general c.i.t.s.

In the case $G=\mathbb{R}^{n}$, the structure of continuous one-parameter semigroups in $M^{1}(G)$ was obtained by P. Lévy, and his work has led to the following description of the relation between continuous convolution semigroups and their discrete approximations; we have the following equivalent properties:

(a) $\mu$ is the limit of a c.i.t.s.

(b) $\mu$ is embeddable.

(c) $\mu$ is infinitely divisible, i.e. it has an $n$th root for all $n \in \mathbb{N}$.

One can refer to $[\mathrm{F}$ (pp. 294, 550) for a proof, in particular for a Fourier analytic proof of the equivalence of $(\mathrm{a})$ and $(\mathrm{b})$ on the real line and to $\mathrm{L}$ (chap. 5) for a different proof.

For a Lie group $G$, the structure of one-parameter convolution semi-groups on $G$ was obtained by $\mathrm{G}$. A. Hunt in $[\mathrm{Hu}]$ and extended to the locally compact setting in $[\mathrm{H}]$. The problem of full extension of Lévy's results to a Lie group was considered there, as well as in subsequent works, in particular [W], Ca], [Fe, Ga], $\mathrm{PRaV}$, Si], [StV] and the book $\mathrm{Gr}$. It was observed in [Hu that equivalence of (a) and (b) cannot hold in general without the commutativity condition in the definition of c.i.t.s. On the other hand, using real algebraic group techniques, the equivalence of (b) and (c) for connected linear groups has been shown in [DM]. Here we are interested in the relation between (a) and (b), and we show its equivalence under some conditions. Our method is based on the algebraic approach to this class of 
problems initiated in $[\mathrm{Ke}]$ and further developed in [RuzS], in the general framework of Delphic semigroups, and on a detailed group theoretic analysis.

In order to state our results we fix some notations. We denote by $Z$ the center of $G$, and by $H^{0}$ the connected component of the identity in a subgroup $H \subset G$. We denote by $\hat{G}$ the real vector space of continuous homomorphisms of $G$ into the additive group of $\mathbb{R}$. For $\mu \in M^{1}(G)$, we denote by $G_{\mu}$ the closed subgroup generated by the support of $\mu$ and $Z_{\mu}=\left\{g \in G \mid g \mu g^{-1}=\mu\right\}$. Furthermore, $\mu$ is said to be symmetric (resp. normal) if $\mu=\tilde{\mu}$ (resp. $\mu \tilde{\mu}=\tilde{\mu} \mu$ ), where $\tilde{\mu}$ is defined by $\tilde{\mu}(X)=\mu\left(X^{-1}\right)$ for any Borel set $X \subset G$. We say that $\Delta=\left(\mu_{i j}\right)_{i \in \mathbb{N}, j=1}^{n_{i}}$ in $M^{1}(G)$ is symmetric (resp. normal) if each $\mu_{i j}$ is symmetric (resp. normal). We denote by $F_{\mu}$ the set of two-sided factors of $\mu$, i.e. the elements $\alpha \in M^{1}(G)$ such that for some $\alpha^{\prime} \in M^{1}(G), \alpha * \alpha^{\prime}=\alpha^{\prime} * \alpha=\mu$. For any bounded complex measure $\lambda$ and any complex function $f$ on $G, \lambda(f)$ will denote, when convenient, the integral of $f$ with respect to $\lambda$, i.e. $\lambda(f)=\int f(g) \mathrm{d} \lambda(g)$.

We consider the space $L^{2}(G)$ of square integrable complex functions on $G$ with respect to a left Haar measure, and the left regular representation $\rho$ of $G$. For $\lambda \in M^{1}(G)$, we denote by $\rho(\lambda)=\int \rho(g) \mathrm{d} \lambda(g)$ the operator on $L^{2}(G)$ corresponding to $\lambda$, and $r(\lambda)$ will denote its spectral radius. We say that $\mu$ has finite 1-moment if for every positive Borel sub-additive function $\delta$ on $G$, we have $\mu(\delta)<\infty$. If $V \subset G$ is a compact neighbourhood of $e$, such that $G=\bigcup_{n \geq 0} V^{n}$, and $\delta_{V}(g)=\inf \{n \in$ $\left.\mathbb{N} \cup\{0\} \mid g \in V^{n}\right\}$, this condition is equivalent to $\mu\left(\delta_{V}\right)<\infty$ (cf. G2 ). The subsemigroup of elements in $M^{1}(G)$ which satisfy this condition will be denoted by $M^{1, m}(G)$.

As special cases of locally compact groups we shall consider subgroups of $\mathbb{F}$ rational points of Zariski connected algebraic groups defined over a local field $\mathbb{F}$, i.e. a finite algebraic extension of either the real field $\mathbb{R}$, or the field $\mathbb{Q}_{p}$ of $p$-adic numbers or the field $\mathbb{F}_{p}((x))$ of Laurent power series over the finite field $\mathbb{F}_{p}$ of $p$ elements, (where $p$ is a prime). We know (see [Ma], p. 56) that such a group $G$ is Zariski dense in the corresponding given algebraic group G. For any subgroup $H \subset G$ we denote by $Z^{c}(H)\left(\right.$ resp. $\left.\mathbf{Z}^{\mathbf{c}}(\mathbf{H})\right)$ the Zariski closure of $H$ in $G$ (resp. $\mathbf{G}$ ), i.e. the set of zeros of polynomials with coefficients in $\mathbb{F}$ which vanish on $H$ (cf. [Bo]).

Theorem 1.1. Let $\mu$ be the limit of a c.i.t.s. $\Delta$ on a locally compact group $G$ such that $Z_{\mu}^{0} / Z^{0}$ is compact. Suppose any one of the following conditions is satisfied:

(1) $\operatorname{supp} \mu$ is contained in a closed subgroup $L_{\mu} \subset G$ which is a compact extension of a closed solvable normal subgroup of $L_{\mu}$.

(2) $\Delta$ is normal.

Then there exist a semigroup $\Sigma=\left\{\mu_{t}\right\}_{t \geq 0}, \mu_{0}=\omega_{H}$, and a compact subgroup $C \subset Z_{\mu}$ such that $\mu$ is quasi-embeddable in $\Sigma$ relative to $C$. Each $\mu_{t}$ is normal if $\Delta$ is normal. Moreover, if $C / H$ is arcwise connected, then $\mu$ itself is embeddable.

Remark. In a compact connected abelian group $G$ which is not arcwise connected, (for e.g. the $p$-adic solenoid), any element $x$ which is outside the arc-component of the identity is infinitesimally divisible, infinitely divisible but not embeddable.

Theorem 1.2. Suppose $G$ is a locally compact group and $\mu$ is the limit of a symmetric c.i.t.s. Then $\mu$ is embeddable. 
In the case where the c.i.t.s. $\Delta=\left(\mu_{i j}\right)_{i \in \mathbb{N}, j=1}^{n_{i}}\left(n_{i} \rightarrow \infty\right)$ has equal components, i.e. if $\mu_{i j}=\mu_{i}, \mu_{i} \rightarrow \delta_{e}, \Delta$ converges to $\mu$ if $\mu_{i}^{n_{i}} \rightarrow \mu$, we have the following theorem.

Theorem 1.3. Suppose $\mu$ is the limit of a c.i.t.s. with equal components on a locally compact group $G$. If $Z_{\mu}^{0} / Z^{0}$ is compact, then there exist a semigroup $\Sigma=\left\{\mu_{t}\right\}_{t \geq 0}$, $\mu_{0}=\omega_{H}$, and a compact subgroup $C \subset Z_{\mu}$ such that $\mu$ is quasi-embeddable in $\Sigma$ relative to $C$. Moreover, if every compact connected subgroup of $Z_{\mu}^{0}$ is arcwise connected, then $\mu$ itself is embeddable.

In particular, if $\mu$ is infinitely divisible with arbitrarily small roots, then $\mu$ is quasi-embeddable; and it is embeddable if the underlying group is a Lie group or totally disconnected group.

The following two theorems are special cases of Theorem 1.1 for Lie groups and F-algebraic groups.

Theorem 1.4. Let $\mu$ be the limit of a c.i.t.s. on a Lie group or totally disconnected group $G$ such that $\operatorname{supp} \mu$ is contained in a closed subgroup $L_{\mu}$ which is a compact extension of a closed solvable normal subgroup. If $Z_{\mu}^{0} / Z^{0}$ is compact, then $\mu$ is embeddable. In particular, if $\mu$ is the limit of a c.i.t.s. on a Zariski connected $\mathbb{F}$ algebraic group, $G_{\mu}$ is amenable and for $\mathbb{F}=\mathbb{R}, Z^{c}\left(G_{\mu}\right)=G$, then $\mu$ is embeddable.

The following special cases of the above result were already known: $G$ compact (cf. [Ca] $), G$ connected nilpotent (cf. [Sh3]). The first part of the above was known if $G$ is totally disconnected (cf. [Sh3] ).

Theorem 1.5. Let $\mu$ be the limit of a normal c.i.t.s. on a Lie group or totally disconnected group $G$. If $Z_{\mu}^{0} / Z^{0}$ is compact, then $\mu$ is embeddable. In particular, if $G$ is a Zariski connected $\mathbb{F}$-algebraic group and for $\mathbb{F}=\mathbb{R}, Z^{c}\left(G_{\mu}\right)=G$, then $\mu$ is embeddable.

In the ultrametric situation in Theorems 1.4 and 1.5 the condition $Z^{c}\left(G_{\mu}\right)=G$ is not necessary because $Z_{\mu}^{0}=Z^{0}=\{e\}$. The condition that $Z_{\mu}^{0} / Z^{0}$ is compact is a "fullness" condition on supp $\mu$ which is satisfied for example if $G$ is $\mathbb{R}$-algebraic and $Z^{c}\left(G_{\mu}\right)=G$ (cf. [DM], Theorem 3.2) or if $G$ is totally disconnected; the above result for the Lie group case does not hold without this condition (see section 9).

In the case $G$ is an abelian Lie group, normality is satisfied and the result corresponds (in a stronger form) to Theorem 5.2 in ( $[\mathrm{P}$, p. 96). For $G$ semisimple and systems of isotropic distributions on the associated symmetric space, normality is also satisfied and it corresponds to Theorem 7.2 in [Ga].

In the case $G$ is a countable subgroup of a connected Lie group or of $G L(n, \mathbb{F})$, the hypotheses of normality can be removed (see [Sh3] and Corollary [6.3). For not necessarily normal c.i.t.s. on non-amenable groups we have the following results.

Theorem 1.6. Suppose $\mu$ is the limit of a c.i.t.s. on a Zariski connected $\mathbb{F}$-algebraic group $G$ such that $\mu$ has a density, $Z^{c}\left(G_{\mu}\right)=G$ and, if $\mathbb{F}$ has positive characteristic, then the radical of $\mathbf{G}$ is $\mathbb{F}$-defined. Then $\mu$ is embeddable.

The above theorem is also valid for a real Lie group with finitely many connected components if the condition $Z^{c}\left(G_{\mu}\right)=G$ is replaced by the condition that $Z_{\mu}^{0} / Z^{0}$ is compact (see the Remark at the end of section 8).

Theorem 1.7. Suppose $\mu$ is the limit of a c.i.t.s. on a Zariski connected $\mathbb{F}$-algebraic group $G, \mu$ has finite 1-moment and $Z^{c}\left(G_{\mu}\right)=G$. Then $\mu$ is embeddable. 
For the proofs of the above theorems we construct multiplicative functions on closed subsets of $M^{1}(G)$ which are continuous at $\delta_{e}$ with respect to weak topology. This gives results of independent interest. For example, we have the following results, the first one being the multiplicativity property for spectral radius.

Theorem 1.8. Suppose $\mu, \nu \in M^{1}(G)$ are normal and commute with each other. Then $r(\mu * \nu)=r(\mu) r(\nu)$ and $\lim _{\nu \rightarrow \delta_{e}} r(\mu * \nu)=r(\mu)$.

Suppose $\mathbf{G}$ is a semisimple algebraic group which is Zariski connected and defined over the local field $\mathbb{F}$. We fix a maximal $\mathbb{F}$-split torus $\mathbf{T}$ in $\mathbf{G}$ and a basis $\Pi=$ $\left\{\alpha_{1}, \ldots, \alpha_{r}\right\}$ of the restricted root system of $\mathbf{G}$. We denote by $\mathbf{P}$ the minimal parabolic subgroup defined by $\Pi$ (see $[\underline{\mathrm{Bo}})$ ), by $\mathbf{Z}_{\mathbf{T}}$ the centraliser of $\mathbf{T}$ in $\mathbf{G}$, by $\mathbf{U}$ the unipotent radical of $\mathbf{P}$, and by $G, T, P, Z_{T}, U$ the corresponding subgroups of $\mathbb{F}$-points, so that we have $P=Z_{T} \cdot U$, a semidirect product. Every $\mathbb{F}$-character of $\mathbf{Z}_{\mathbf{T}}$ defines an element $\hat{\chi}$ of $\hat{Z}_{T}$ through the formula

$$
\hat{\chi}(z)=\log |\chi(z)| \quad\left(z \in Z_{T}\right)
$$

and these elements form a lattice in $\hat{Z}_{T}$. Hence, if $\mathcal{A}$ is the dual vector space of $\hat{Z}_{T}$, then for every $z \in Z_{T}$, a vector $\hat{z} \in \mathcal{A}$ is defined through the above formula and the kernel of the map $z \mapsto \hat{z}$ is the maximal compact subgroup $Z_{c}$ of $Z_{T}$ (see [T1]). We fix a scalar product of $\mathcal{A}$ which is invariant under the action of the restricted Weyl group of $\mathbf{G}$, we denote by $\mathcal{A}^{+} \subset \mathcal{A}$ the Weyl chamber defined by $\widehat{\Pi} \subset \widehat{Z}_{T}$ and by $\hat{\mathcal{A}}^{+} \subset \mathcal{A}$ the orthogonal cone of $\mathcal{A}^{+}$, and we consider the order on $\mathcal{A}$ defined by $\hat{\mathcal{A}}^{+}$. From [T1] we know that there exists a maximal compact subgroup $K$ of $G$ containing $Z_{c}$ such that the following Iwasawa and polar decompositions are valid:

$$
G=K Z_{T} U, \quad G=K \overline{Z_{T}^{+}} K
$$

where $Z_{T}^{+}$is the inverse image of $\mathcal{A}^{+}$in $Z_{T}$. If $z(g, k)$ and $z(g)$ are components in $Z_{T}, \overline{Z_{T}^{+}}$of $g k, g$ respectively, then the vectors $H(g, k)=\hat{z}(g, k) \in \mathcal{A}, H(g)=$ $\hat{z}(g) \in \mathcal{A}$ are well defined. Furthermore, $H(g, k)=H(g, x)$ depends only on $g$ and the projection $x$ of $k$ in the flag space $\mathcal{F}=G / P$. For any $\alpha \in M^{1, m}(G)$ and $\eta \in M^{1}(\mathcal{F})$ we also write $H(\alpha, \eta)=\int H(g, x) \mathrm{d} \alpha(g) \mathrm{d} \eta(x)$ and we denote by $\mathcal{S}(\alpha) \subset M^{1}(\mathcal{F})$ the set of $\alpha$-stationary measures, i.e. probability measures $\eta$ such that $\alpha * \eta=\int g \cdot \eta \mathrm{d} \alpha(g)=\eta$. An element $g \in G$ is said to be proximal if its action on $G / P$ has a unique fixed attracting non-neutral point $x \in G / P$, i.e. there exists a co-dimension one algebraic submanifold $V \subset G / P$ such that $x \notin V, g V \subset V$, and for every $y \notin V, \lim _{n} g^{n} \cdot y=x$.

Theorem 1.9. Suppose $G$ is the group of $\mathbb{F}$-rational points of an algebraic group $\mathbf{G}$ defined over a local field $\mathbb{F}$, which is semisimple and Zariski connected and let $\alpha \in M^{1, m}(G)$. Then with the above notations we have the following:

(1) The limit $L(\alpha)=\lim _{n} \frac{1}{n} \int H(g) \mathrm{d} \alpha^{n}(g)$ exists and belongs to $\overline{\mathcal{A}^{+}}$.

(2) $L(\alpha)=\sup \{H(\alpha, \eta) \mid \eta \in \mathcal{S}(\alpha)\}$.

(3) If $\alpha, \beta \in M^{1, m}(G)$ commute with each other, then $L(\alpha)+L(\beta)-L(\alpha * \beta) \in$ $\hat{\mathcal{A}}^{+}$.

(4) If $\mu \in M^{1, m}(G)$ satisfies $Z^{c}\left(G_{\mu}\right)=G$ and if $\alpha$ commutes with $\mu$, then $L(\alpha)=H(\alpha, \eta)$ for any $\eta \in \mathcal{S}(\alpha)$. In particular, if $\alpha, \beta \in F_{\mu}$ commute with each other, then $L(\alpha)+L(\beta)=L(\alpha * \beta)$.

(5) If $\mu$ is as above, the map $\alpha \mapsto L(\alpha)$ from $F_{\mu}$ to $\overline{\mathcal{A}^{+}}$is continuous. 
(6) The condition $L(\alpha)=0$ is equivalent to the condition that $G_{\alpha}$ is amenable and $\alpha(\log |f|)=0$ for any algebraic $\mathbb{F}$-character $f$ of $Z^{c}\left(G_{\alpha}\right)$.

(7) If $Z^{c}\left(G_{\alpha}\right)=G$, then the condition that $L(\alpha) \in \mathcal{A}^{+}$is equivalent to the fact that the semigroup generated by $\operatorname{supp} \alpha$ contains a proximal element.

We explain in the next section the main lines of our approach. In sections 34 , we develop constructions of partial homomorphisms. In section 5 , we prove the statements from section 2 , which are crucial for the proofs. In section 6 , we construct suitable semigroups in the amenable case. In sections $7-9$, we give proofs, complements, examples and counterexamples.

\section{PARTIAL hOMOMORPHiSMS AND DIVISIBILITy WITH RESPECT TO CONVOLUTION SEMIGROUPS}

Our approach is based on the properties of a family of closed abelian subsemigroups (with identity) of $M^{1}(G)$ or its quotients. For such a semigroup $S$ with identity $e$, and $s \in S$, we denote by $F_{s}$ the set of two-sided factors of $s$ in $S$. For a closed subset $X \subset S$, with $e \in X$, we denote by $X^{*}$ the set of multiplicative partial homomorphisms of $X$ into $[0,1]$, which are continuous at $e$. Hence $f \in X^{*}$ is equivalent to $f(s t)=f(s) f(t)$ if $s, t, s t \in X$ and $\lim _{s_{n} \rightarrow e} f\left(s_{n}\right)=1$. Here, two properties of $F_{s}$ are essential for us. The first one is the boundedness of the number of factors in appropriate decompositions of $s$, which is closely related to compactness of $F_{s}$ modulo invertible elements (cf. Lemma 5.1 and [Sh3]), and is a consequence of the study of concentration functions (cf. [DSh], JRoW]). The second one is the existence of a non-trivial and non-vanishing element in $F_{s}^{*}$. Here we consider a c.i.t.s. converging to $\mu$ and we need to construct a "nice" abelian semigroup $S$ in which $\mu$ is infinitesimally divisible, and then to get, using properties of $S$, a continuous oneparameter semigroup $\left\{\mu_{t}\right\}_{t>0} \subset S$ containing $\mu$. The construction of $S$ depends on the first property; the existence of $\left\{\mu_{t}\right\}_{t \geq 0}$ depends also on it, as well as on the construction of non-trivial elements in $F_{s}^{*}$ or $X^{*}$, where $X \subset F_{s}$. Here, we use the spectral methods of $\left[\mathrm{DeG}\right.$ and $\left[\mathrm{BC}\right.$, Gelfand's theory of $C^{*}$ Banach algebras and then the boundary theoretical methods of $[\mathrm{Fu}]$. Then we develop the ideas of $\mathrm{Ke}$ and $\mathrm{Ruz}$ as well as the techniques of [Sh3], but we modify the definitions of [RuzS] in order to fit with the setting of sub-semigroups of $M^{1}(G)$ and their quotients. Let us recall some definitions.

Let $\mu, \nu \in M^{1}(G)$ and let $x \in G$. We will sometimes use $\mu \nu$ instead of $\mu * \nu$ for the convolution product of $\mu$ and $\nu$, let $x \mu=\delta_{x} * \mu$ and we identify $x$ with $\delta_{x}$ and any set $X$ of $G$ with the set $\left\{\delta_{x} \mid x \in X\right\}$. Let supp $\mu$ denote the support of $\mu$ in $G$ and let $I(\mu)=\{x \in G \mid x \mu=\mu x=\mu\}$. Then $I(\mu)$ is a compact subgroup of $Z_{\mu}$. If $H$ is a compact subgroup, we denote by $\omega_{H}$ its normalised Haar measure, and let $M_{H}^{1}(G)=\omega_{H} M^{1}(G) \omega_{H}$, the semigroup of $H$-bi-invariant probability measures $\mu$, i.e. $H \subset I(\mu)$.

Let $S$ be a Hausdorff semigroup with identity $e$ and let $s \in S$. Let $F_{s}$ (in $S$ ) denote the set of two sided factors of $s$, that is, $F_{s}=\{t \in S \mid t r=r t=$ $s$ for some $r \in S\}$. An element $h \in S$ is said to be an idempotent if $h^{2}=h$. A subset $X$ of $S$ is said to be idempotent-free (in $S$ ) if $X$ does not contain any idempotent except possibly identity $e$. Elements $x, y$ of $S$ are said to be associates if $x \in F_{y}$ and $y \in F_{x}$. A subset $X$ is said to be associate-free if $X$ does not contain any two elements which are associates. We denote by $S^{u}$ the subgroup of units, i.e. the invertible elements, in $S$. Also, if $S$ is abelian, we consider the congruence 
relation $(\approx) \bmod S^{u}$, we denote by $\xi$ the canonical map of $S$ onto $S_{\xi}=S / S^{u}$, and for brevity we write $\xi(s)=\bar{s}$ for all $s \in S$. In the case $S \subset M^{1}(G)$, the congruence relation $(\sim) \bmod Z^{0}$ will also be considered. In that case we denote by $\pi$ the canonical map from $S$ onto $S_{\pi}=S / Z^{0}$. Note that both $\xi$ and $\pi$ are continuous open homomorphisms. A decomposition of $s$ as $s=s_{1} \cdots s_{n}$, for some $n \in \mathbb{N}$, where $s_{i} \in X$ and $s_{i} s_{j}=s_{j} s_{i}$ for all $i, j$, is called an $X$-decomposition of $s$. Suppose $Y \subset X$ and suppose $s$ has a $Y$-decomposition $s=\prod_{i=1}^{n} \prod_{j=1}^{m_{i}} t_{i j}$. Then it is said to be finer than the above $X$-decomposition if $\prod_{j=1}^{m_{i}} t_{i j}=s_{i}$ for each $i$. An element $s \in S$ is said to be infinitesimally divisible if $s$ has a $U$-decomposition for every neighbourhood $U$ of $e$ in $S$. A commutative infinitesimal triangular system (c.i.t.s.) in $S$ is defined in the same way as above. A decomposition $s=s_{1} \cdots s_{n}$ is said to be $X$-closed if $\prod_{i=1}^{m} s_{\alpha(i)} \in X$, for all permutations $\alpha$ of $\{1, \ldots, n\}$ and for all $m \leq n$.

Definition. Let $S$ be a Hausdorff first countable abelian semigroup with identity $e$ and let $s \in S$. Let $\left\{U_{n}\right\}_{n \in \mathbb{N}}$ be a neighbourhood basis of $e$ in $S$ such that $U_{n+1} \subset U_{n}$ for all $n$. Let $X \subset F_{s}$. Then $s$ is said to be $\left\{U_{n}\right\}$-tree-like divisible in $X$ if $s$ has a sequence of $U_{n}$-decompositions which are $X$-closed and are finer and finer (i.e. for each $n$, the $U_{n+1}$-decomposition is finer than the $U_{n}$-decomposition above).

Clearly, any element which is $\left\{U_{n}\right\}$-tree-like divisible in any set $X$ is infinitesimally divisible. Conversely, if $s \in S$ is such that every $\alpha \in F_{s}$ is infinitesimally divisible, then $s$ is $\left\{U_{n}\right\}$-tree-like divisible in $F_{s}$ for any neighbourhood basis $\left\{U_{n}\right\}$ of $e$ as above.

Definition. Let $S$ be a Hausdorff abelian semigroup with identity $e$ and let $s \in$ $S \backslash\{e\}$. Let $F(s)$ be a closed subset of $F_{s}$ such that $e, s \in F(s)$. Let $f: F(s) \rightarrow \mathbb{R}^{+}$. We say that $f$ is an $s$-norm on $F(s)$ if

(1) $f$ is continuous at $e$ and $f(s)>0$,

(2) $f\left(s_{1} s_{2}\right)=f\left(s_{1}\right)+f\left(s_{2}\right)$ if $s_{1}, s_{2}, s_{1} s_{2} \in F(s)$

(i.e. $f$ is a partial homomorphism, $e^{-f} \in[F(s)]^{*}, e^{-f}>0$ and $e^{-f}(s)<1$ ).

The following results are fundamental in our approach. The first two results are generalisations of Statement 8.2 in [Ruz] and Theorem 2.3 in [Sh2], respectively.

Theorem 2.1. Let $S$ be a Hausdorff abelian first countable semigroup with identity e. Let $\left\{U_{n}\right\}_{n \in \mathbb{N}}$ be a neighbourhood basis of e in $S$ such that $U_{n+1} \subset U_{n}$ for every $n$. Let $s \in S \backslash\{e\}$. Suppose there exists a set $F(s) \subset F_{s}$ such that:

(1) $e, s \in F(s)$ and $F(s)$ is compact, associate-free and idempotent-free.

(2) $s$ is $\left\{U_{n}\right\}$-tree-like divisible in $F(s)$.

(3) There exists an s-norm on $F(s)$.

Then $s$ has a non-trivial square factor, i.e. $s=v^{2} v^{\prime}$ for some $v \in F(s) \backslash\{e\}$, $v^{\prime} \in F_{s}$.

The proof of the above theorem in section 5 is somewhat similar to that of Statement 8.2 in $[\mathrm{Ruz}$ except for some crucial modifications needed for the present situation. One has to use the set $F(s)$ instead of $F_{s}$ and use the decomposition given by condition (2) in the hypothesis instead of infinitesimal divisibility. This generalisation is used in proving the first part of Theorem 1.1, where the original result of $[\mathrm{Ruz}]$ does not apply. 
Corollary 2.2. Let $S$ be a Hausdorff abelian semigroup with identity e. Let $s \in$ $S \backslash\{e\}$ be such that $F_{s}$ is compact, associate-free and idempotent-free. If for every $v \in F_{s} \backslash\{e\}$ there exists some $r \in F_{v} \backslash\{e\}$ and a subset $F(r)$ such that conditions of the above theorem are satisfied by $r$ and $F(r)$, then there exists a continuous one-parameter semigroup $\left\{s_{t}\right\}_{t \geq 0}$ in $S$ such that $s_{1}=s$.

The proof is essentially the same as the proof of Ruzsa's Theorem (cf. [Ruz, Theorem on p. 118) using Theorem 2.1. So we will not repeat it here.

The following is the basic theorem which allows us to pass from limits of infinitesimal triangular systems to infinitesimal divisibility inside a "nice" semigroup $S$.

Theorem 2.3. Let $G$ be a locally compact group. Let $\mu$ be the limit of a c.i.t.s. and suppose $Z_{\mu}^{0} / Z^{0}$ is compact. Then there exists a compact subgroup $H \subset I(\mu)$, a closed abelian semigroup $S \subset M_{H}^{1}(G)$ with identity $h=\omega_{H}$ such that $Z^{0} h \subset S$, $\mu \in S$, and

(1) any $\alpha$ in $F_{\mu}$ (in $S$ ) is infinitesimally divisible in $S$,

(2) $F_{\pi(\mu)}$ is compact in $\pi(S)$ and idempotent-free,

(3) the subgroup $S^{u}$ of units in $S$ is connected and $S^{u} / Z^{0} h$ is compact.

Moreover, if $\mu$ is the limit of a c.i.t.s. with equal components, then $\mu=\lim _{n \rightarrow \infty} \lambda_{n}^{k_{n}}$ for some sequence $\left\{\lambda_{n}\right\} \subset S$, such that $\pi\left(\lambda_{n}\right) \rightarrow \pi(h)$.

Also, if the c.i.t.s. is normal, then $S$ can be chosen such that $S$ consists of normal measures.

\section{Construction of partial homomorphism USING SPECTRAL METHODS}

As in the Introduction, we consider the Hilbert space $L^{2}(G)$ of square integrable complex functions with respect to the left Haar measure $\mathrm{d} g$ on $G$ and the regular representation by left translation on $G: \rho(g)(f)(x)=f\left(g^{-1} x\right)$ for any $f \in L^{2}(G)$ and any $g, x \in G$. If $\nu \in M(G)$, the Banach algebra of bounded complex measures, let $\rho(\nu)$ be the operator defined by $\rho(\nu)(f)=\int_{G} \rho(g) f \mathrm{~d} \nu(g)$; hence $\rho(\nu)$ is a bounded linear operator on $L^{2}(G)$, i.e. $\rho(\nu) \in \mathcal{L}\left[L^{2}(G)\right]$. Moreover, $\nu \mapsto \rho(\nu)$ defines a one-to-one complex Banach algebra homomorphism from $M(G)$ to $\mathcal{L}\left[L^{2}(G)\right]$ with $\|\rho(\nu)\| \leq\|\nu\|$. It is well known that for any $\mu \in M^{1}(G), r(\mu)=1$ if and only if $G_{\mu}$ is amenable (cf. [DeG], BC]). Until Theorem [3.6, we are mainly interested in the case when $G_{\mu}$ is non-amenable, since otherwise the following statements are obvious.

In order to give the proof of Theorem 1.8, we introduce a new notation and establish some lemmas.

If $V$ is a compact symmetric neighbourhood of the identity $e$ in $G$, then we define $\delta(g)=\delta_{V}(g)=\inf \left\{n \in \mathbb{N} \cup\{0\} \mid g \in V^{n}\right\}$ if $g \in \bigcup_{n=0}^{\infty} V^{n}$ and $\delta(g)=\infty$ otherwise. Then $\delta\left(g g^{\prime}\right) \leq \delta(g)+\delta\left(g^{\prime}\right)$, i.e. $\delta$ is sub-additive, $\delta\left(g^{-1}\right)=\delta(g)$ and $e^{-\delta}$ is super-multiplicative.

Lemma 3.1. With the above notation, there exists $\gamma>0$ such that $e^{-\gamma \delta_{V}} \in L^{2}(G)$.

Proof. For any $\gamma>0$, we have

$$
\left\|e^{-\gamma \delta_{V}}\right\|_{2}^{2}=\sum_{n=1}^{\infty} e^{-2 \gamma n}\left(\left|V^{n}\right|-\left|V^{n-1}\right|\right),
$$


where $\left|V^{k}\right|$ is the left Haar measure of $V^{k}$, for any $k$. From [G1], we know that $\lim _{n \rightarrow \infty}\left|V^{n}\right|^{1 / n}=c<\infty$. Hence, for some $k>0$ and every $n,\left|V^{n}\right| \leq k(c+\epsilon)^{n}$, where $\epsilon>0$. It follows that

$$
\sum_{n=1}^{\infty} e^{-2 \gamma n}\left(\left|V^{n}\right|-\left|V^{n-1}\right|\right) \leq k \sum_{n=1}^{\infty} e^{-2 \gamma n}(c+\epsilon)^{n}<\infty \text { if } 2 \gamma>c+\epsilon .
$$

This implies that for $\gamma>c / 2,\left\|e^{-\gamma \delta_{V}}\right\|_{2}^{2}<\infty$.

Lemma 3.2. If $\mu, \nu \in M^{1}(G)$, then $\mu * \nu\left(e^{-\delta}\right) \geq \mu\left(e^{-\delta}\right) \nu\left(e^{-\delta}\right)$.

Proof. We have $e^{-\delta}(x y) \geq e^{-\delta}(x) e^{-\delta}(y)$. Hence

$$
\mu * \nu\left(e^{-\delta}\right)=\int e^{-\delta(x y)} \mathrm{d} \mu(x) \mathrm{d} \nu(y) \geq \int e^{-\delta(x)} \mathrm{d} \mu(x) \int e^{-\delta(y)} \mathrm{d} \nu(y) .
$$

Lemma 3.3. Suppose $\gamma \in \mathbb{R}^{+}$is such that $e^{-\gamma \delta_{V}} \in L^{2}(G)$. Then

$$
r(\mu) \geq \varlimsup_{n}\left[\tilde{\mu}^{n} * \mu^{n}\left(e^{-\gamma \delta_{V}}\right)\right]^{1 / 2 n} \geq \lim _{n \rightarrow \infty}\left[\mu^{n}\left(e^{-\gamma \delta_{V}}\right)\right]^{1 / n} \geq \mu\left(e^{-\gamma \delta_{V}}\right) .
$$

Proof. We denote $f=e^{-\gamma \delta_{V}}=e^{-\delta}$. Then

$$
\begin{aligned}
\left\|\mu^{n} * f\right\|_{2}^{2} & =\left\langle\tilde{\mu}^{n} * \mu^{n} * f, f\right\rangle \\
& =\int e^{-\delta\left(g^{-1} x\right)-\delta(x)} \mathrm{d}\left(\tilde{\mu}^{n} * \mu^{n}\right)(g) \mathrm{d} x \\
& \geq \int e^{-\delta(y)} \mathrm{d}\left(\tilde{\mu}^{n} * \mu^{n}\right)(y) \int e^{-2 \delta(x)} \mathrm{d} x \\
& =\tilde{\mu}^{n} * \mu^{n}\left(e^{-\delta}\right)\left\|e^{-\delta}\right\|_{2}^{2} .
\end{aligned}
$$

Hence

$$
r(\mu) \geq \varlimsup_{n}\left\|\mu^{n} * f\right\|_{2}^{1 / n} \geq \varlimsup_{n}\left[\tilde{\mu}^{n} * \mu^{n}\left(e^{-\delta}\right)\right]^{1 / 2 n} .
$$

The last inequality follows from Lemma 3.2 since $\tilde{\mu}^{n}\left(e^{-\delta}\right)=\mu^{n}\left(e^{-\delta}\right)$ and $\left[\mu^{n}\left(e^{-\delta}\right)\right]^{1 / n}$ converges to its supremum.

Lemma 3.4. Suppose $\mu$ is normal. Then for any $\psi \in C_{c}^{+}(G)$,

$$
r(\mu)=\lim _{n \rightarrow \infty}\left[\tilde{\mu}^{n} * \mu^{n}(\psi)\right]^{1 / 2 n} .
$$

Proof. It is proved in Theorem 1 in [BC] that if $\nu$ is symmetric and positive definite (i.e. $\langle\nu * \phi, \phi\rangle \geq 0$ for every $\phi \in C_{c}(G)$ ), then $\lim _{n}\left[\nu^{n}(\psi)\right]^{1 / n}=r(\nu)$, for every $\psi \in C_{c}^{+}(G)$. Hence it suffices to take $\nu=\mu * \tilde{\mu}$ and to observe that $\mu^{n} * \tilde{\mu}^{n}=(\mu * \tilde{\mu})^{n}$ and $r(\mu * \tilde{\mu})=[r(\mu)]^{2}$ since $\mu$ is normal.

Lemma 3.5. Suppose $\gamma \in \mathbb{R}^{+}$is such that $e^{-\gamma \delta_{V}} \in L^{2}(G)$. Then for $\mu$ normal, we have $r(\mu)=\lim _{n \rightarrow \infty}\left[\tilde{\mu}^{n} * \mu^{n}\left(e^{-\gamma \delta_{V}}\right)\right]^{1 / 2 n}$.

Proof. We choose $\psi \in C_{c}^{+}(G)$, such that $\psi \leq e^{-\gamma \delta_{V}}$. Then we obtain from Lemma 3.4

$$
\lim _{n \rightarrow \infty}\left[\tilde{\mu}^{n} * \mu^{n}\left(e^{-\gamma \delta_{V}}\right)\right]^{1 / 2 n} \geq \lim _{n \rightarrow \infty}\left[\tilde{\mu}^{n} * \mu^{n}(\psi)\right]^{1 / 2 n}=r(\mu) .
$$

On the other hand, from Lemma 3.3 .

$$
r(\mu) \geq \varlimsup_{n}\left[\tilde{\mu}^{n} * \mu^{n}\left(e^{-\gamma \delta_{V}}\right)\right]^{1 / 2 n} .
$$


Proof of Theorem 1.8. We observe that $\mu * \tilde{\mu}$ and $\nu * \tilde{\nu}$ are symmetric. Moreover, since $\mu$ and $\nu$ commute and are normal, it is known that $\mu * \nu$ is normal and $\mu * \tilde{\mu}$ and $\nu * \tilde{\nu}$ commute. Also, $r(\lambda)=r(\lambda * \tilde{\lambda})^{1 / 2}$ for any normal measure $\lambda$. Therefore, without loss of generality we may assume that $\mu$ and $\nu$ are symmetric. In particular, $r(\mu)=\|\rho(\mu)\|, r(\nu)=\|\rho(\nu)\|$ and hence

$$
r(\mu * \nu)=\|\rho(\mu * \nu)\| \leq\|\rho(\mu)\|\|\rho(\nu)\|=r(\mu) r(\nu) .
$$

On the other hand, if $f=e^{-\gamma \delta_{V}}$ belongs to $L^{2}(G)$, by Lemma 3.5 we get that $r(\mu)=\lim _{n \rightarrow \infty}\left[\mu^{2 n}(f)\right]^{1 / 2 n}$. Therefore, using Lemma 3.2, we get

$$
r(\mu * \nu)=\lim _{n \rightarrow \infty}\left[\mu^{2 n} * \nu^{2 n}(f)\right]^{1 / 2 n} \geq \lim _{n \rightarrow \infty}\left[\mu^{2 n}(f) \nu^{2 n}(f)\right]^{1 / 2 n}=r(\mu) r(\nu) .
$$

Theorem 3.6. If $\mu \in M^{1}(G)$, then $r(\mu)>0$. Suppose $\left\{\mu_{n}\right\} \subset M^{1}(G)$ converges weakly to $\mu \in M^{1}(G)$ and $\mu$ is normal. Then $\lim _{n \rightarrow \infty} r\left(\mu_{n}\right) \geq r(\mu)$. In particular, if $G_{\mu}$ is amenable, then $\lim _{n \rightarrow \infty} r\left(\mu_{n}\right)=r(\mu)=1$.

Proof. From Lemma 3.1 we fix $\gamma>0$ with $e^{-\gamma \delta_{V}} \in L^{2}(G)$. From the proof of Lemma 3.3. we know that for any $n>0$,

$$
r(\mu) \geq\left[\tilde{\mu}^{n} * \mu^{n}\left(e^{-\gamma \delta_{V}}\right)\right]^{1 / 2 n} c_{n}, \quad \text { where } \quad c_{n}=\left\|e^{-\gamma \delta_{V}}\right\|_{2}^{1 / n} .
$$

Since $e^{-\gamma \delta_{V}}$ is continuous at $e$ and also strictly positive near $e$, we have $\tilde{\mu}^{n} *$ $\mu^{n}\left(e^{-\gamma \delta_{V}}\right)>0$ and hence $r(\mu)>0$.

We have as above, for every $k$,

$$
r\left(\mu_{n}\right) \geq\left[\left(\tilde{\mu}_{n}^{k} * \mu_{n}^{k}\right)\left(e^{-\gamma \delta_{V}}\right)\right]^{1 / 2 k} c_{k} .
$$

Hence

$$
\lim _{n \rightarrow \infty} r\left(\mu_{n}\right) \geq \lim _{n \rightarrow \infty}\left[\left(\tilde{\mu}_{n}^{k} * \mu_{n}^{k}\right)\left(e^{-\gamma \delta_{V}}\right)\right]^{1 / 2 k} c_{k}=\left[\left(\tilde{\mu}^{k} * \mu^{k}\right)\left(e^{-\gamma \delta_{V}}\right)\right]^{1 / 2 k} c_{k} .
$$

Since $\mu$ is normal, using Lemma 3.5.

$$
\lim _{n \rightarrow \infty} r\left(\mu_{n}\right) \geq \lim _{k \rightarrow \infty}\left[(\tilde{\mu} * \mu)^{k}\left(e^{-\gamma \delta_{V}}\right)\right]^{1 / 2 k}=r(\tilde{\mu} * \mu)^{1 / 2}=r(\mu) .
$$

If $G_{\mu}$ is amenable, then $r(\mu)=1$, hence $1 \geq \lim _{n \rightarrow \infty} r\left(\mu_{n}\right) \geq r(\mu)=1$. In particular, $\lim _{n \rightarrow \infty} r\left(\mu_{n}\right)=r(\mu)$.

Corollary 3.7. On a locally compact group $G$, suppose $\mu$ belongs to a commutative semigroup $S$ consisting of normal measures with identity $\omega_{H}$ and $G_{\mu}$ is nonamenable. Then the map $\nu \mapsto-\log r(\nu)$ from $S$ to $\mathbb{R}^{+}$gives a $\mu$-norm on $F_{\mu}$.

However, in the more general case where $G_{\mu}$ is arbitrary, we have the following:

Theorem 3.8. Suppose $G$ is a locally compact group, $S$ is a closed commutative semigroup of normal measures in $M_{H}^{1}(G)$ with identity $\omega_{H}$. If $\mu \in S$ is not a translate of an idempotent, then there exists a $\mu$-norm on $F_{\mu}$.

Proof. We consider the regular representation $\rho$ of $G$ into $\mathcal{L}\left[L^{2}(G)\right]$ and the $C^{*}$ subalgebra $A \subset \mathcal{L}\left[L^{2}(G)\right]$ generated by $S$. We recall that $\rho$ is injective on $M(G)$. We denote by $\Gamma$ the Gelfand spectrum of the $C^{*}$-subalgebra $A$ of $\mathcal{L}\left[L^{2}(G)\right]$ and, for $\nu \in S, \hat{\nu} \in C(\Gamma)$ will denote the Gelfand transform of $\rho(\nu)$, i.e. for every $\gamma \in \Gamma$, $\hat{\nu}(\gamma)=\gamma[\rho(\nu)]$. For any fixed $\psi \in L^{2}(G), \nu \in S,\langle\nu * \psi, \psi\rangle$ extends to a continuous positive linear form on $A$, hence defines a positive Radon measure $\theta_{\psi}$ on $C(\Gamma)$ by the formula $\theta_{\psi}(\hat{\nu})=\langle\nu * \psi, \psi\rangle=\int \hat{\nu}(\gamma) \mathrm{d} \theta_{\psi}(\gamma)$. Since $\theta_{\psi}(1)=\langle\psi, \psi\rangle=1, \theta_{\psi}$ is 
a probability measure on $\Gamma$ (the spectral measure of $\psi$ ). Moreover, since $\rho(\nu)$ has norm at most 1 , we have $|\hat{\nu}(\gamma)| \leq 1$.

Since $\tilde{\mu} * \mu$ is not an idempotent, there exists $\gamma_{0} \in \Gamma$ with $0<\left|\hat{\mu}\left(\gamma_{0}\right)\right|<1$. Hence from the continuity of $\hat{\mu}(\gamma)$, we can find an open set $U \subset \Gamma$ and an $\epsilon>0$ such that

$$
\forall \gamma \in U, \epsilon<|\hat{\mu}(\gamma)|<1-\epsilon \text { and } \epsilon<-\log |\hat{\mu}(\gamma)|<-\log \epsilon<\infty .
$$

Since every $u \in C^{+}(\Gamma)$ has a square root in $C^{+}(\Gamma)$ which corresponds to a non-zero hermitian operator $a$ in $A$, there exists $\phi \in L^{2}(G)$ with $\theta_{\phi}(u)=\left\langle a^{2} \phi, \phi\right\rangle>0$. It follows that there exists $\psi \in L^{2}(G),\|\psi\|_{2}=1$, with $\theta_{\psi}(U)>0$. On the other hand, if $\nu \in F_{\mu}$ and $\gamma \in U$, we have the basic relation

$$
\epsilon<|\hat{\mu}(\gamma)| \leq|\hat{\nu}(\gamma)| \leq 1
$$

In particular, since $\omega_{H} \in F_{\mu}$, we have $\hat{\omega}_{H}(\gamma)=1$ for all $\gamma \in U$. Hence

$$
\forall \gamma \in U, 0 \leq-\log |\hat{\nu}(\gamma)| \leq-\log |\hat{\mu}(\gamma)|<-\log \epsilon .
$$

Then, since $-\log |\hat{\nu}(\gamma)|$ is continuous on $U$, we can define a functional $f$ on $F_{\mu}$ by the formula

$$
f(\nu)=\int_{U}-\log |\hat{\nu}(\gamma)| \mathrm{d} \theta_{\psi}(\gamma)
$$

Clearly, $f(\mu)>\epsilon \theta_{\psi}(U)>0$. The additivity of $f$ on $F_{\mu}$ follows from the multiplicativity of the map $\nu \mapsto \hat{\nu}(\gamma)$ and the definition of $f(\nu)$.

In order to show the continuity of $f$ at $\omega_{H}$, suppose $\nu_{n} \in F_{\mu}$ converges to $\omega_{H}$. We observe that since $|\hat{\nu}(\gamma)|^{2}$ is between $\epsilon^{2}$ and 1 , we have

$$
-\log \left|\hat{\nu}_{n}(\gamma)\right|^{2} \leq \frac{1}{\epsilon^{2}}\left[1-\left|\hat{\nu}_{n}(\gamma)\right|^{2}\right], \text { for all } \gamma \in U
$$

Hence

$$
\begin{aligned}
f\left(\nu_{n}\right) & \leq \frac{1}{2 \epsilon^{2}} \int_{U}\left[\hat{\omega}_{H}(\gamma)-\left|\hat{\nu}_{n}(\gamma)\right|^{2}\right] \mathrm{d} \theta_{\psi}(\gamma), \\
f\left(\nu_{n}\right)^{2} & \leq C^{2} \int\left[\hat{\omega}_{H}(\gamma)-\left|\hat{\nu}_{n}(\gamma)\right|^{2}\right]^{2} \mathrm{~d} \theta_{\psi}(\gamma),
\end{aligned}
$$

where $C=\frac{1}{2 \epsilon^{2}} \theta_{\psi}(U)^{1 / 2}$. On the other hand, for $\alpha_{n}=\left(\tilde{\nu}_{n} * \nu_{n}-\omega_{H}\right)^{2}$, by definition

$$
\theta_{\psi}\left(\hat{\alpha}_{n}\right)=\int\left[\hat{\omega}_{H}(\gamma)-\left|\hat{\nu}_{n}(\gamma)\right|^{2}\right]^{2} \mathrm{~d} \theta_{\psi}(\gamma)=\left\langle\left(\tilde{\nu}_{n} * \nu_{n}-\omega_{H}\right)^{2} * \psi, \psi\right\rangle .
$$

From the weak convergence of $\tilde{\nu}_{n} * \nu_{n}$ to $\omega_{H}$, we get the convergence of the above expression to zero, hence $\lim _{n \rightarrow \infty} f\left(\nu_{n}\right)=0$.

Remark. The above construction is reminiscent of the construction of a so-called Khinchine functional in the setting of a locally compact abelian group $G$ through the formula

$$
f(\nu)=\int_{U}-\log |\hat{\nu}(\gamma)| \mathrm{d} \gamma
$$

where $\hat{\nu}$ is the Fourier transform of $\nu$ and $U$ is a suitable neighbourhood of zero in the dual group of $G$. 


\section{Construction of partial homomorphisms USING BOUNDARY THEORY}

Here, as in the Introduction, $G$ is the group of $\mathbb{F}$-rational points of a semisimple Zariski connected algebraic group defined over a local field $\mathbb{F}$. Let $S$ be a commutative semigroup of $M^{1}(G)$ with identity $\omega_{H}$, and let $\mu \in S$ be such that $Z^{c}\left(G_{\mu}\right)=G$. Below we give constructions of elements of $F_{\mu}^{*}$ in two special cases: when $\mu$ has a density or when $\mu$ has finite 1-moment.

\section{a) When $\mu$ has a density}

We denote by $m=\mathrm{d} x$ the unique $K$-invariant measure on $\mathcal{F}=G / P$ and we consider the corresponding Hilbert space $L^{2}(\mathcal{F})$. In this situation we use the unitary representation $\rho_{0}$ of $G$ on $L^{2}(\mathcal{F})$ defined by

$$
\rho_{0}(g) \phi(x)=\left[\frac{\mathrm{d} g \cdot m}{\mathrm{~d} m}\right]^{1 / 2}(x) \phi\left(g^{-1} x\right),
$$

where $\phi \in L^{2}(\mathcal{F}), x \in \mathcal{F}, g \in G$. Clearly $\rho_{0}$ is unitary and for any $\lambda \in M^{1}(G)$, the operator $\rho_{0}(\lambda)$ defined by

$$
\rho_{0}(\lambda)=\int \rho_{0}(g) \mathrm{d} \lambda(g)
$$

is bounded and $\left\|\rho_{0}(\lambda)\right\| \leq\|\lambda\|$. Let $r_{0}(\lambda)$ denote the spectral radius of $\rho_{0}(\lambda)$.

If $\lambda$ has a density, it is a limit of measures $\lambda_{n}$ with continuous densities with compact support. For such measures, $\rho_{0}\left(\lambda_{n}\right)$ is given by a continuous kernel on $\mathcal{F} \times \mathcal{F}$, hence is a compact operator. Since $\lim _{n}\left\|\rho_{0}\left(\lambda_{n}\right)-\rho_{0}(\lambda)\right\|=0$, the same is true of $\rho_{0}(\lambda)$.

Theorem 4.1. Suppose $S$ is a commutative semigroup in $M^{1}(G)$ and $\mu \in S$ satisfies, for some $n \in \mathbb{N}$,

$$
\mu^{n}=c \omega_{H}+\mu_{n, H},
$$

where $H$ is a compact subgroup of $I(\mu), c \in\left[0,1\left[\right.\right.$ and $\mu_{n, H}$ has a positive density. Then there exists a continuous homomorphism $f$ of $S$ into $[0,1]$ such that

$$
f(\alpha) \leq r(\alpha), \quad \text { and } \quad 0<f(\mu)=r(\mu)<1 .
$$

For the proof we need the following lemma.

Lemma 4.2. With the above notations, we denote $\mu_{n, H}=\mu_{n}$, for $r=r\left(\mu_{n}\right)$, $\mathcal{H}_{r}=\operatorname{ker}\left[\rho_{0}\left(\mu_{n}\right)-r \mathrm{Id}\right]$, and $\mathcal{H}_{r}^{\prime}=\operatorname{ker}\left[\rho_{0}\left(\mu^{n}\right)-(r+c) \mathrm{Id}\right]$. Then we have

$$
0<\operatorname{dim} \mathcal{H}_{r}<\infty, \quad \mathcal{H}_{r}=\mathcal{H}_{r}^{\prime} \subset \operatorname{ker}\left[\rho_{0}\left(\omega_{H}\right)-\mathrm{Id}\right]
$$

and

$$
r(\mu)=\left[c+r\left(\mu_{n}\right)\right]^{1 / n} .
$$

Proof. We know that $Z_{T} / T$ is compact (cf. [Ma, p. 54), hence $P=Z_{T} \cdot U$ is amenable. Then it follows that $r_{0}\left(\mu_{n}\right)=r\left(\mu_{n}\right)$ (cf. G2], p. 92), hence from Theorem 3.6 that $r_{0}\left(\mu_{n}\right)>0$.

From above we know that $\rho_{0}\left(\mu_{n}\right)$ is a compact operator in $L^{2}(\mathcal{F})$. On the other hand, $\rho_{0}\left(\mu_{n}\right)$ preserves the cone $L_{+}^{2}(\mathcal{F})$ of positive functions in $L^{2}(\mathcal{F})$, hence from a classical theorem of Krein-Rutmann (see [KRut]), there exists $\phi \in L_{+}^{2}(\mathcal{F})$ such that $\rho_{0}\left(\mu_{n}\right) \phi=r_{0}\left(\mu_{n}\right) \phi$. Hence we have, using the compactness of $\rho_{0}\left(\mu_{n}\right)$,

$$
0<\operatorname{dim} \mathcal{H}_{r}<\infty .
$$


Since $H \subset I(\mu)$, we have that $\omega_{H} * \mu_{n}=\mu_{n} * \omega_{H}=\mu_{n}$. It follows that $\rho_{0}\left(\omega_{H}\right)$ preserves $\mathcal{H}_{r}$ and that its restriction to $\mathcal{H}_{r}$ is the identity operator. Hence

$$
\mathcal{H}_{r} \subset \operatorname{ker}\left[\rho_{0}\left(\omega_{H}\right)-\mathrm{Id}\right] .
$$

Therefore, $\rho_{0}\left(\mu^{n}\right)$ also preserves $\mathcal{H}_{r}$ and it acts on $\mathcal{H}_{r}$ as $(r+c)$ Id, i.e. we have $\mathcal{H}_{r} \subset \mathcal{H}_{r}^{\prime}$. The same reasoning is valid for $\rho_{0}\left(\mu_{n}\right)$ acting on $\mathcal{H}_{r}^{\prime}$, hence $\mathcal{H}_{r}^{\prime} \subset \mathcal{H}_{r}$. So we have

$$
\mathcal{H}_{r}=\mathcal{H}_{r}^{\prime} \subset \operatorname{ker}\left[\rho_{0}\left(\omega_{H}\right)-\mathrm{Id}\right] .
$$

Since $\rho_{0}\left(\omega_{H}\right)$ and $\rho_{0}\left(\mu_{n}\right)$ commute, we have $r_{0}\left(\mu^{n}\right) \leq c r_{0}\left(\omega_{H}\right)+r_{0}\left(\mu_{n}\right)$, and hence $r_{0}\left(\mu^{n}\right) \leq c+r_{0}\left(\mu_{n}\right)$. Since $\mathcal{H}_{r}^{\prime} \neq\{0\}$, we also have $r_{0}\left(\mu^{n}\right) \geq c+r$. Hence $r_{0}\left(\mu^{n}\right)=c+r$. Finally, $r_{0}(\mu)=(c+r)^{1 / n}=r(\mu)$, i.e. $r(\mu)=\left[c+r\left(\mu_{n}\right)\right]^{1 / n}$.

Proof of Theorem 4.1. We consider for any $\alpha \in S$ the operator $\rho_{0}(\alpha)$. Since $\alpha$ commutes with $\mu, \rho_{0}(\alpha)$ leaves $\mathcal{H}_{r}^{\prime}$ invariant, where $\mathcal{H}_{r}^{\prime}$ is as in the lemma above. Hence its restriction to $\mathcal{H}_{r}^{\prime}, \rho_{0}^{(r)}(\alpha)$ is well defined as well as its determinant since $\left.\operatorname{dim} \mathcal{H}_{r}^{\prime}=p \in\right] 0, \infty\left[\right.$. We set $f(\alpha)=\left|\operatorname{det} \rho_{0}^{(r)}(\alpha)\right|^{1 / p}$. Then $f(\mu)=r(\mu)$. Since absolute values of eigenvalues of $\rho_{0}^{(r)}(\alpha)$ are dominated by $r_{o}(\alpha)=r(\alpha)$, we have $f(\alpha) \leq r(\alpha)$. It is clear that $f$ is multiplicative and maps $S$ into $[0,1]$. Also, $0<$ $f(\mu)=r(\mu)<1$, as $G$ is semisimple and $\mu$ has density and hence $\left.\left.f\left(F_{\mu}\right) \subset\right] 0,1\right]$.

\section{b) When $\mu$ has finite 1-moment}

We complete the notations set down before. Observe that since $\mathbf{Z}_{\mathbf{T}}$ is reductive we have $\mathbf{Z}_{\mathbf{T}}=\mathbf{Z}_{\mathbf{T}}^{\prime} \cdot \mathbf{T}$, where $\mathbf{Z}_{\mathbf{T}}^{\prime}$ is the derived group of $\mathbf{Z}_{\mathbf{T}}$ and $\mathbf{Z}_{\mathbf{T}}^{\prime} \cap \mathbf{T}$ is finite (cf. [Bo] $)$. Hence there exists $r \geq 0$ such that, for any character $\chi$ of $\mathbf{T}, \chi^{r}$ also defines a character of $\mathbf{Z}_{\mathbf{T}}$. In particular, $\log |\chi|$ also defines an element of $\hat{Z}_{T}$ which we again denote by $\hat{\chi}$. Then the elements $\hat{\alpha}_{i}$ form a basis of $\hat{Z}_{T}$ and the Weyl chamber $\mathcal{A}^{+}$in $\mathcal{A}$ is defined by the conditions $\hat{\alpha}_{i}(x)>0(1 \leq i \leq r)$. We also consider the dual basis $\hat{\alpha}_{i}^{\prime}(1 \leq i \leq r)$ of $\hat{\alpha}_{i}$ defined by $\left\langle\hat{\alpha}_{i}, \hat{\alpha}_{j}^{\prime}\right\rangle=\delta_{i j}$. For $a, b \in \mathcal{A}$, we write $a \prec b$ if $b-a \in \hat{\mathcal{A}}^{+}$, i.e. $\hat{\alpha}_{i}^{\prime}(a) \leq \hat{\alpha}_{i}^{\prime}(b)$, for all $i \in\{1, \ldots, r\}$. From [T2], we know that for any $i \in\{1, \ldots, r\}$ there exists an irreducible representation $\rho_{i}$ of $\mathbf{G}$ into the $\mathbb{F}$ vector space $V_{i}$ with highest weight $\omega_{i}$ such that $\hat{\omega}_{i}$ is proportional to $\hat{\alpha}_{i}^{\prime}$. Furthermore the weight-space of $\mathbf{T}$ defined by $\omega_{i}$ is one-dimensional and the other weights of $\mathbf{T}$ on $V_{i}$ are of the form $\omega_{i}-\sum_{j=1}^{r} n_{j} \alpha_{j}$ with $n_{j} \in \mathbb{N} \cup\{0\}$. We denote by $e_{i}$ a highest weight vector in $V_{i}$. Such a representation $\rho_{i}$ will be said to be fundamental. It follows that we can put a norm on $V_{i}$ in such a way that

$$
\left\|e_{i}\right\|=1, \quad\left\|\rho_{i}(z)\right\|=\left\|z e_{i}\right\|=\left|\omega_{i}(z)\right|
$$

for any $z \in \bar{Z}_{T}^{+}$and $K$ acts by isometries on $V_{i}$ (while $T$ acts by diagonal matrices).

Since $P=Z_{T} U$ stabilises the line $\mathbb{F} e_{i}$ we have the formula

$$
\left\|\rho_{i}(g k) e_{i}\right\|=\left|\omega_{i}[z(g, k)]\right|, \quad\left\|\rho_{i}(g)\right\|=\left|\omega_{i}[z(g)]\right| .
$$

For the proof of Theorem 1.9 we need some lemmas and the following proposition which summarises in appropriate notations some known facts (see [GR1, GR2]).

Proposition 4.3. For any $g, g^{\prime} \in G$, we have

$$
H\left(g g^{\prime}\right) \prec H(g)+H\left(g^{\prime}\right) .
$$


Suppose $V$ is a compact neighbourhood of e in $G$ and denote $\delta_{V}(g)=\inf \{n \in$ $\left.\mathbb{N} \cup\{0\}, g \in V^{n}\right\}$. Then there exists $c, c^{\prime}>0$ such that

$$
c \delta_{V}(g) \leq H(g) \leq c^{\prime} \delta_{V}(g) .
$$

For any $\alpha \in M^{1, m}(G)$ the following limit exists and belongs to $\overline{\mathcal{A}^{+}}$:

$$
L(\alpha)=\lim _{n} \frac{1}{n} \int H(g) \mathrm{d} \alpha^{n}(g) .
$$

For any fundamental representation $\rho_{i}$ with highest weight $\omega_{i}$ we have

$$
\hat{\omega}_{i}[L(\alpha)]=\lim _{n} \frac{1}{n} \int \log \left\|\rho_{i}(g)\right\| \mathrm{d} \alpha^{n}(g) .
$$

Proof. By the definition of the order on $\mathcal{A}$, formula (1) follows from the relations

$$
\hat{\omega}_{i}\left[H\left(g g^{\prime}\right)\right] \leq \hat{\omega}_{i}[H(g)]+\hat{\omega}_{i}\left[H\left(g^{\prime}\right)\right] \quad(1 \leq i \leq r) .
$$

From above, this can be written as

$$
\log \left\|\rho_{i}\left(g g^{\prime}\right)\right\| \leq \log \left\|\rho_{i}(g)\right\|+\log \left\|\rho_{i}\left(g^{\prime}\right)\right\|
$$

and this follows from sub-additivity of the map $g \mapsto \log \left\|\rho_{i}(g)\right\|$. From the equivalence of norms on $\mathcal{A}$ we have, for some constant $d>0$,

$$
\forall z \in Z, \quad\|\hat{z}\| \leq d \sum_{i=1}^{r}\left|\hat{\omega}_{i}(\hat{z})\right| .
$$

Hence for any $g \in G$, from above,

$$
\|H(g)\| \leq d \sum_{i=1}^{r} \log \left\|\rho_{i}(g)\right\| .
$$

Since, for any $i,\left\|\rho_{i}(g)\right\|$ is subadditive and $G=\bigcup_{n \geq 0} V^{n}$, there exists $c_{i}>0$ such that

$$
\log \left\|\rho_{i}(g)\right\| \leq c_{i} \delta_{V}(g) .
$$

Hence $\|H(g)\| \leq \sup _{1 \leq i \leq r} r d c_{i} \delta_{V}(g)$.

On the other hand, we can choose $V$ to be of the form $V=K V_{+} K$, where $V_{+}=\left\{z \in \bar{Z}^{+} \mid\|\hat{z}\| \leq 1\right\}$ and then $V^{n} \supset K V_{+}^{n} K=\{g \in G \mid\|H(g)\| \leq n\}$. It follows that $\delta_{V}(g) \leq\|H(g)\|$. Hence the relation (2) is valid with $c=1$ and $c^{\prime}=\sup _{1 \leq i \leq r} r d c_{i}$.

The property $\alpha * \beta \in M^{1, m}(G)$ if $\alpha, \beta \in M^{1, m}(G)$ follows from the sub-additivity of the function $\delta(g)=\|H(g)\|$ considered above, hence the integral $\int H(g) \mathrm{d} \alpha^{n}(g)$ is finite.

By convexity of $\overline{\mathcal{A}^{+}}$, we have $\int H(g) \mathrm{d} \alpha^{n}(g) \in \overline{\mathcal{A}^{+}}$. Furthermore, formula (1) implies the sub-additivity of the sequence $n \mapsto \int H(g) \mathrm{d} \alpha^{n}(g)$, hence the required convergence toward $L(\alpha) \in \overline{\mathcal{A}^{+}}$follows.

From above,

$$
\hat{\omega}_{i}[H(g)]=\log \left\|\rho_{i}(g)\right\|,
$$

and formula (4) follows clearly from the definitions, integration and taking the limit.

Lemma 4.4. There exists a constant $c>0$ such that for any $g \in G$,

$$
\left\|\int H(g, x) \mathrm{d} x-H(g)\right\| \leq c .
$$


Proof. By definition, it suffices to show that, for any fundamental weight $\omega_{i}(1 \leq$ $i \leq \operatorname{dim} A), \int \hat{\omega}_{i}[H(g, k)] \mathrm{d} k-\hat{\omega}_{i}[H(g)]$ is bounded. With the above notations, we have $\left|\omega_{i}[z(g, k)]\right|=\left\|\rho_{i}(g) \rho_{i}(k) e_{i}\right\|$ and

$$
\left|\omega_{i}[z(g)]\right|=\left\|\rho_{i}[z(g)] e_{i}\right\|=\left\|\rho_{i}(g)\right\| .
$$

Hence $\hat{\omega}_{i}[H(g, k)] \leq \hat{\omega}_{i}[H(g)]$ and hence $H(g, k) \prec H(g)$. Then it suffices to show that

$$
\inf _{g \in G} \int \log \left\|\rho_{i}(g) \rho_{i}(k) e_{i}\right\| \mathrm{d} k-\log \left\|\rho_{i}(g)\right\|>-\infty .
$$

We denote by $\theta$ the image of measure $\mathrm{d} k$ under the map $k \mapsto \rho_{i}(k) e_{i}=v$. The above relation can be written as

$$
\inf _{g \in G} \int \log \left\|\rho_{i}(g) v\right\| \mathrm{d} \theta(v)-\log \left\|\rho_{i}(g)\right\|>-\infty .
$$

Clearly $\theta$ is $K$-invariant and has support equal to the algebraic submanifold $Q_{i}=$ $\rho_{i}(G) e_{i}$ of $P\left(V_{i}\right)$ which is the image of $K$ by the map $k \mapsto \rho_{i}(k) e_{i}$.

Suppose that the above inequality is not true. Then for some sequence $\left\{g_{n}\right\} \subset G$, we have

$$
\lim _{n} \int \log \left\|\left(\rho_{i}\left(g_{n}\right) /\left\|\rho_{i}\left(g_{n}\right)\right\|\right) v\right\| \mathrm{d} \theta(v)=-\infty .
$$

We can find $c_{n} \in \mathbb{F}$ and choose $g_{n} \in G$ such that $\left\{c_{n} \rho_{i}\left(g_{n}\right)\right\}$ converges to a diagonal map $\tau \in$ End $V_{i}$ such that $\|\tau\| \geq 1$ and then $\int \log \|\tau v\| \mathrm{d} \theta(v)=-\infty$. On the other hand, since the set $\left\{v \in Q_{i} \mid\|\tau v\|=0\right\}$ is an algebraic submanifold of $Q_{i}$ with codimension $N \geq 1$ and since $\theta$ is the natural measure on $Q_{i}$, there exists a constant $c>0$ such that for any $t>0$

$$
\phi(t)=\theta\left\{v \in Q_{i} \mid\|\tau v\|<t\right\} \leq c t^{N} .
$$

Hence (using charts on $Q_{i}$ and the homogeneity of the Haar measure on $\mathbb{F}$ )

$$
\begin{aligned}
\int \log \|\tau v\| \mathrm{d} \theta(v) & =\int_{0}^{\|\tau\|} \log t \mathrm{~d} \phi(t) \\
& =\phi(\|\tau\|)-\int_{0}^{\|\tau\|} \phi(t) \frac{\mathrm{d} t}{t} \\
& \geq-\int_{0}^{\|\tau\|} t^{N-1} \mathrm{~d} t \\
& =-\frac{1}{N}\|\tau\|^{N}>-\infty \text { since } \quad N>0 .
\end{aligned}
$$

This leads to a contradiction, hence the assertion follows.

Lemma 4.5. Suppose $\alpha, \beta \in M^{1, m}(G)$ commute. Then

$$
L(\alpha * \beta) \prec L(\alpha)+L(\beta) .
$$

Proof. From above, we know that $\alpha * \beta \in M^{1, m}(G)$, hence $L(\alpha * \beta)$ and $L\left[(\alpha * \beta)^{n}\right]$ are well defined. But here, $(\alpha * \beta)^{n}=\alpha^{n} * \beta^{n}$, hence from Proposition 4.3, we get that

$$
\begin{aligned}
\int H(g) \mathrm{d}(\alpha * \beta)^{n}(g) & =\int H\left(g g^{\prime}\right) \mathrm{d} \alpha^{n}(g) \mathrm{d} \beta^{n}\left(g^{\prime}\right) \\
& \prec \int H(g) \mathrm{d} \alpha^{n}(g)+\int H\left(g^{\prime}\right) \mathrm{d} \beta^{n}\left(g^{\prime}\right) .
\end{aligned}
$$

In the limit: $L(\alpha * \beta) \prec L(\alpha)+L(\beta)$. 
Proposition 4.6. For any $\alpha \in M^{1, m}(G)$, we have

$$
L(\alpha)=\sup _{\eta \in \mathcal{S}(\alpha)} H(\alpha, \eta)
$$

and the supremum (with respect to $\hat{\mathcal{A}}^{+}$) is attained. Furthermore, if $Z^{c}\left(G_{\alpha}\right)=G$, then for any $\eta \in \mathcal{S}(\alpha)$, we have that $L(\alpha)=H(\alpha, \eta)$ and the sequence

$$
\frac{1}{n} \int H(g, x) \mathrm{d} \alpha^{n}(g)
$$

converges to $L(\alpha)$ uniformly in $x \in \mathcal{F}$.

Proof. As observed in the proof of Lemma 4.4, we have $H(g, x) \prec H(g)$, hence for any $\eta \in \mathcal{S}(\alpha)$,

$$
H\left(\alpha^{n}, \eta\right) \prec \alpha^{n}(H), \quad H(\alpha, \eta)=\lim _{n} \frac{1}{n} H\left(\alpha^{n}, \eta\right) \prec \lim _{n} \frac{1}{n} \alpha^{n}(H)=L(\alpha) .
$$

Conversely, we can construct a measure $\eta \in \mathcal{S}(\alpha)$ such that $L(\alpha)=H(\alpha, \eta)$ as follows. We denote $S_{n}=g_{n} \cdots g_{1}$. From the cocycle property of $H$, for any $\alpha \in M^{1, m}(G)$, we get

$$
\begin{gathered}
H\left(S_{n}, x\right)=\sum_{k=1}^{n} H\left(g_{k}, S_{k-1} \cdot x\right), \\
\frac{1}{n} \int H(g, x) \mathrm{d} \alpha^{n}(g)=H\left(\alpha, \alpha_{n} * \delta_{x}\right), \text { where } \alpha_{n}=\frac{1}{n} \sum_{k=1}^{n-1} \alpha^{k} .
\end{gathered}
$$

Since $\alpha \in M^{1, m}(G)$, the function $h(y)=\int H(g, y) \mathrm{d} \alpha(g)$ is continuous and we have with $\theta=\mathrm{d} x$,

$$
\frac{1}{n} H\left(\alpha^{n}, \theta\right)=\left(\alpha_{n} * \theta\right)(h) .
$$

From the sequence $\left\{\alpha_{n} * \theta\right\}$ of $M^{1}(\mathcal{F})$ one can extract a convergent subsequence converging to an $\alpha$-stationary measure $\eta$ and

$$
\lim _{n} \frac{1}{n} H\left(\alpha^{n}, \theta\right)=\eta(h)
$$

From Lemma 4.4, we have

$$
\left\|H\left(\alpha^{n}, \theta\right)-\alpha^{n}(H)\right\| \leq c .
$$

Hence, by the definition of $L$,

$$
L(\alpha)=\lim _{n} \frac{1}{n} H\left(\alpha^{n}, \theta\right)=\eta(h)=H(\alpha, \eta) .
$$

In order to obtain the second assertion, we show that, for any $i \in\{1, \ldots, r\}$ and for any $\rho_{i}(\alpha)$-stationary probability measure $\lambda$ on $P\left(V_{i}\right)$,

$$
\int \log \left\|\rho_{i}(g) y\right\| \mathrm{d} \alpha(g) \mathrm{d} \lambda(y)=\hat{\omega}_{i}(L(\alpha))=\gamma_{i}(\alpha),
$$

where $\gamma_{i}(\alpha)=\lim _{n} \frac{1}{n} \int \log \left\|\rho_{i}(g)\right\| \mathrm{d} \alpha^{n}(g)$ is the largest characteristic exponent of the random matrix product $\rho_{i}\left[S_{n}(\omega)\right]$. Since the stabiliser of $e_{i} \in P\left(V_{i}\right)$ is a standard parabolic subgroup $P_{i}$ of $G$ (see [B] ), there is a $G$-equivariant map $x \mapsto x_{i}$ 
from $G / P$ onto $G / P_{i} \subset P\left(V_{i}\right)$. We denote by $\eta_{i}$ the corresponding projection of $\eta$ on $P\left(V_{i}\right)$; clearly $\eta_{i}$ is $\rho_{i}(\alpha)$-stationary and, from above,

$$
\hat{\omega}_{i}[H(\alpha, \eta)]=\int \log \left\|\rho_{i}(g) y\right\| \mathrm{d} \eta_{i}(y) \mathrm{d} \alpha(g) .
$$

We consider the product space $G^{\mathbb{N}}$, the shift transformation $\sigma$ on $G^{\mathbb{N}}$, the product measure $\pi=\alpha^{\otimes \mathbb{N}}$ on $G^{\mathbb{N}}$, and we use the multiplicative ergodic theorem for $\rho_{i}\left[S_{n}(\omega)\right],\left(\omega \in G^{\mathbb{N}}\right)$ (see $[\underline{\mathrm{R}})$. Since $\pi$ is $\sigma$-invariant with

$$
\int \log \left\|\rho_{i}\left[S_{1}(\omega)\right]\right\| \mathrm{d} \pi(\omega)<\infty \text { and } \int \log \left\|\rho_{i}\left[S_{1}^{-1}(\omega)\right]\right\| \mathrm{d} \pi(\omega)<\infty,
$$

this theorem implies the existence of a $\sigma$-invariant Borel set $\Omega \subset G^{\mathbb{N}}$ such that $\pi(\Omega)=1$ and for any $y \in V_{i}$,

$$
\lim _{n} \frac{1}{n} \log \left\|\rho_{i}\left[S_{n}(\omega)\right] y\right\|=J(\omega, y)
$$

where $J(\omega, y)$ has value equal to one of the characteristic exponents of $\rho_{i}\left[S_{n}(\omega)\right]$, hence is bounded by $\gamma_{i}(\alpha)$. Also, this convergence takes place in $L^{1}(\pi)$, hence it follows that

$$
\lim _{n} \frac{1}{n} \int \log \left\|\rho_{i}(g) y\right\| \mathrm{d} \alpha^{n}(g)=\int J(\omega, y) \mathrm{d} \pi(\omega) \leq \gamma_{i}(\alpha) .
$$

Furthermore, from the first part, there exists $\eta \in \mathcal{S}(\alpha)$ with $H(\alpha, \eta)=L(\alpha)$, hence $\gamma_{i}(\alpha)=\hat{\omega}_{i}[H(\alpha, \eta)]=\int \log \left\|\rho_{i}(g) y\right\| \mathrm{d} \alpha(g) \mathrm{d} \eta_{i}(y)$, and for any $n \in \mathbb{N}$ and $y \in V_{i}$, we have

$$
\begin{aligned}
J(\omega, y) \leq \gamma_{i}(\alpha) & =\frac{1}{n} \int \log \left\|\rho_{i}(g) y\right\| \mathrm{d} \alpha^{n}(g) \mathrm{d} \eta_{i}(y) \\
& =\lim _{n} \frac{1}{n} \int \log \left\|\rho_{i}(g) y\right\| \mathrm{d} \alpha^{n}(g) \mathrm{d} \eta_{i}(y) \\
& =\int J(\omega, y) \mathrm{d} \pi(\omega) \mathrm{d} \eta_{i}(y) .
\end{aligned}
$$

It follows that

$$
\pi \otimes \eta_{i}-\text { a.e. }: J(\omega, y)=\gamma_{i}(\alpha) .
$$

In particular, there exists $v_{0} \in V_{i}$ such that

$$
\pi-\text { a.e. }: J\left(\omega, v_{0}\right)=\gamma_{i}(\alpha) .
$$

We consider the set

$$
V_{i}^{-}=\left\{v \in V_{i} \mid \pi\left\{\omega \mid J(\omega, v)<\gamma_{i}(\alpha)\right\}=1\right\} .
$$

By definition $J\left(\omega, v+v^{\prime}\right) \leq \sup \left\{J(\omega, v), J\left(\omega, v^{\prime}\right)\right\}$ and $J(\omega, \lambda v)=J(\omega, v)$ for any $\lambda \in \mathbb{F}$, hence $V_{i}^{-}$is a subspace of $V_{i}$. Furthermore, the condition $J\left(\sigma \omega, g_{1}(\omega) v\right)=$ $J(\omega, v)$ and the fact that $\pi$ is a product measure implies that $V_{i}^{-}$is invariant under the action of the support of $\rho_{i}(\alpha)$. Since $Z^{c}\left(G_{\alpha}\right)=G, \rho_{i}$ is irreducible and $V_{i}^{-} \neq V_{i}$, it follows that $V_{i}^{-}=\{0\}$. On the other hand, if $\theta \in M^{1}\left[P\left(V_{i}\right)\right]$ is $\rho_{i}(\alpha)$-stationary and satisfies $\int \log \left\|\rho_{i}(g) v\right\| \mathrm{d} \alpha(g) \mathrm{d} \theta(v)<\gamma_{i}(\alpha), \theta$ can be supposed to be ergodic and then the Birkhoff ergodic theorem implies that

$$
\theta \otimes \pi \text {-a.e. }: J(\omega, v)=\lim _{n} \frac{1}{n} \log \left\|\rho_{i}(g) v\right\|=\int \log \left\|\rho_{i}(g) v\right\| \mathrm{d} \alpha(g) \mathrm{d} \theta(v)<\gamma_{i}(\alpha),
$$


hence $v \in V_{i}^{-}$. In particular, for some $v \in P\left(V_{i}\right), \pi\left\{\omega \mid J(\omega, v)<\gamma_{i}(\alpha)\right\}=1$. Since $V_{i}^{-}=\{0\}$, this is impossible, hence $\int \log \left\|\rho_{i}(g) v\right\| \mathrm{d} \alpha(g) \mathrm{d} \theta(v)=\gamma_{i}(\alpha)$ for any $\theta$ as above.

The first fact of the second assertion already follows from this: for any $\eta \in \mathcal{S}(\alpha)$, we have, for $i \in\{1, \ldots, r\}$,

$$
\hat{\omega}_{i}[H(\alpha, \eta)]=\int \log \left\|\rho_{i}(g) y\right\| \mathrm{d} \alpha(g) \mathrm{d} \eta(y)=\gamma_{i}(\alpha)
$$

since $\eta_{i} \in M^{1}\left[P\left(V_{i}\right)\right]$ is $\rho_{i}(\alpha)$-invariant. Hence $H(\alpha, \eta)=L(\alpha)$.

To get the uniform convergence of $\frac{1}{n} \int H(g, x) \mathrm{d} \alpha^{n}(g)$ toward $L(\alpha)$, we can use the arguments as in the beginning of the proof. We have, with the same notations,

$$
\frac{1}{n} \int H\left(g, x_{n}\right) \mathrm{d} \alpha^{n}(g)=H\left(\alpha, \alpha_{n} * \delta_{x_{n}}\right)
$$

for any sequence $\left\{x_{n}\right\}$ in $\mathcal{F}$. We can always extract convergent subsequences from the bounded sequences $\left\{H\left(\alpha, \alpha_{n} * \delta_{x_{n}}\right)\right\}$ and $\left\{\alpha_{n} * \delta_{x_{n}}\right\}$ and suppose that $\left\{H\left(\alpha, \alpha_{n_{k}} * \delta_{x_{n_{k}}}\right)\right\}$ converges and $\left\{\alpha_{n_{k}} * \delta_{x_{n_{k}}}\right\}$ converges to $\eta \in M^{1}(\mathcal{F})$. Since $x \mapsto \int H(g, x) \mathrm{d} \alpha(g)$ is continuous,

$$
\lim _{k} H\left(\alpha, \alpha_{n_{k}} * \delta_{x_{n_{k}}}\right)=H(\alpha, \eta) .
$$

On the other hand, the relation $\alpha * \alpha_{n}=\alpha_{n}+\frac{1}{n}\left(\alpha^{n}-\delta_{e}\right)$ implies that $\alpha * \eta=\eta$, hence $\eta \in \mathcal{S}(\alpha)$. From above we get

$$
L(\alpha)=\lim _{k} \frac{1}{n_{k}} \int H\left(g, x_{n_{k}}\right) \mathrm{d} \alpha^{n_{k}}(g),
$$

hence the required uniform convergence.

Definition. A representation $\rho$ of a group $L$ on a vector space is said to be totally irreducible if the restriction of $\rho$ to any subgroup of $L$ of finite index is irreducible.

The following is an extension of a well-known lemma of $[\mathrm{Fu}$.

Lemma 4.7. Suppose that $L$ is a locally compact amenable group, $\mathbb{F}$ is a local field and $\varrho$ is a representation of $L$ into a d-dimensional $\mathbb{F}$ vector space $V$. Then, if $\varrho$ is totally irreducible, the ratio $\|\varrho(g)\| /|\operatorname{det} \varrho(g)|^{1 / d}$ is bounded on $L$.

Proof. If possible, suppose there exists a sequence $\left\{g_{n}\right\} \subset L$ such that

$$
\lim _{n} \frac{\left\|\varrho\left(g_{n}\right)\right\|}{\left|\operatorname{det} \varrho\left(g_{n}\right)\right|^{1 / d}}=\infty .
$$

Since, when $\mathbb{F}$ is ultrametric, the absolute values in $\mathbb{F}^{*}=\mathbb{F} \backslash\{0\}$ are the numbers $|\pi|^{n}(n \in \mathbb{Z})$, where $\pi$ is a uniformizer in $\mathbb{F}$, we can find for general $\mathbb{F},\left\{c_{n}\right\} \subset \mathbb{F}$ such that, for all $n$,

$$
\left|c_{n}\right| \leq\left\|\varrho\left(g_{n}\right)\right\| \leq|\pi|\left|c_{n}\right|
$$

We denote by $u_{n}=c_{n}^{-1} \varrho\left(g_{n}\right)$ and observe that

$$
\left|\operatorname{det} u_{n}\right|=\left|c_{n}\right|^{-d}\left|\operatorname{det} \varrho\left(g_{n}\right)\right| \quad \text { and } \quad\left\|u_{n}\right\|=\left|c_{n}\right|^{-1}\left\|\varrho\left(g_{n}\right)\right\| .
$$

Hence, from the above choice of $c_{n}$,

$$
1 \leq\left\|u_{n}\right\| \leq|\pi|, \quad \lim _{n}\left|\operatorname{det} u_{n}\right|=0
$$

We can suppose that $\lim _{n} u_{n}=u \in G L(V)$, hence $\operatorname{det} u=0$. We denote by $D_{1}$ and $D_{2}$ the non-trivial subspaces of $P(V)$, corresponding to $\operatorname{Ker} u, \operatorname{Im} u$. 
Since $L$ is amenable, there exists an $L$-invariant probability measure $\nu$ on $P(V)$, hence

$$
g_{n} \cdot \nu=\nu, \quad \lim _{n} g_{n} \cdot \nu=\nu .
$$

We write $\nu=\nu_{1}+\nu_{2}$, where $\nu_{1}$ (resp. $\nu_{2}$ ) has support in $D_{1}\left(\operatorname{resp} . P(V) \backslash D_{1}\right)$. We denote by $u \cdot x \in D_{2}$ the image of $x \in P(V) \backslash D_{1}$ under the quasi-projective map defined by $u$. We can suppose that the sequence $\left\{g_{n} \cdot \nu_{1}\right\}$ of bounded measures converges to a measure $\nu_{3}$ supported on a proper subspace $D_{3}=\lim _{n} g_{n} \cdot D_{1} \subset$ $P(V)$. Then, from dominated convergence, we have

$$
\lim _{n} g_{n} \cdot \nu_{2}=u \cdot \nu_{2} \text {, hence } \lim _{n} g_{n} \cdot \nu=\nu_{3}+u \cdot \nu_{2} \text {. }
$$

This means that $\nu$ is supported on $D_{2} \cup D_{3}$. Then we can consider $D$, the smallest finite union of projective subspaces such that $\nu(D)=1$, and observe that $D$ is $L$-invariant. This contradicts the total irreducibility of $\varrho$.

The following is an extension to the positive characteristic case of results in [G1].

Lemma 4.8. Suppose $\mathbf{H}$ is an $\mathbb{F}$-algebraic group and $\mathbb{F}^{\prime}$ is a finite algebraic extension of $\mathbb{F}$ such that the radical $\mathbf{R}$ of $\mathbf{H}$ is $\mathbb{F}^{\prime}$-defined and let $H^{\prime}, R^{\prime}$ denote the corresponding subgroups of $\mathbb{F}^{\prime}$-points, respectively. Then, if $L \subset H$ is amenable and $Z^{c}(L)=H$, the group $L R^{\prime} / R^{\prime}$ is relatively compact as a subgroup of $H^{\prime} / R^{\prime}$. In particular, $L$ is contained in a closed subgroup which is a compact extension of a closed solvable normal subgroup.

Proof. Clearly, we can suppose $\mathbf{H}$ to be Zariski connected. We consider a fundamental representation $\rho_{i}$ of the semisimple group $\mathbf{H} / \mathbf{R}$, hence of $\mathbf{H}$ into $V_{i}$. Since $\mathbf{H} / \mathbf{R}$ is connected and $\rho_{i}$ is irreducible, it follows that $\rho_{i}$ is totally irreducible. Since $\mathbf{Z}^{\mathbf{c}}\left[\rho_{\mathbf{i}}(\mathbf{L})\right]=\rho_{\mathbf{i}}(\mathbf{H})$, the restriction of $\rho_{i}$ to $L$ is also totally irreducible. Also, since $\left|\operatorname{det} \rho_{i}(g)\right|=1$ for any $g \in L$, Lemma 4.7 implies that $\rho_{i}(L)$ is bounded.

Since the linear forms $\hat{\omega}_{i}$ generate $\hat{Z}_{T}$, we see that the kernel of the representation $\rho=\bigoplus_{i=1}^{r} \rho_{i}$ is a finite extension of the $\mathbb{F}^{\prime}$-anisotropic component of $\mathbf{H} / \mathbf{R}$. Since the group of $\mathbb{F}^{\prime}$-rational points of this component is compact and the projection of $H^{\prime}$ into the group of $\mathbb{F}^{\prime}$-rational points of $\mathbf{H} / \mathbf{R}$ is closed (see Ma , pp. 50-54), it follows that the kernel of $\rho$ in $H^{\prime} / R^{\prime}$ is compact and the projection of $L$ into $H^{\prime} / R^{\prime}$ is relatively compact.

Lemma 4.9. Let $W$ be a $\mathbb{F}$ vector space, let $G$ be a semisimple subgroup of $G L(W)$ and let $\alpha \in M^{1, m}(G)$. Suppose that $G_{\alpha}$ is amenable and that every $\mathbb{F}$-character $\chi$ of $\mathbf{H}=\mathbf{Z}^{\mathbf{c}}\left(\mathbf{G}_{\alpha}\right)$ satisfies $\alpha(\log |\chi|)=0$. Then

$$
\lim \frac{1}{n} \int \log \|g\| \mathrm{d} \alpha^{n}(g)=0
$$

Proof. We decompose the proof in several steps.

a) Using a finite algebraic extension of $\mathbb{F}$, we can suppose that there exists a decreasing sequence of subspaces $W_{i} \subset W(1 \leq i \leq r+1), W_{1}=W, W_{r+1}=$ $\{0\}$ such that, for every $i \in\{1, \ldots, r\}$, the natural representation $\tau_{i}$ of $\mathbf{H}$ into $W_{i} / W_{i+1}$ is $\mathbb{F}$-irreducible and the unipotent subgroup $\mathbf{U}^{\mathbf{1}}=\operatorname{ker} \bigoplus_{\mathbf{i}=\mathbf{1}}^{\mathbf{r}} \tau_{\mathbf{i}}$ is $\mathbb{F}$-defined and $\mathbb{F}$-split (see $[\mathrm{Bo}]$, p. 205). Then the representation $\tau=\bigoplus_{i=1}^{r} \tau_{i}$ of $\mathbf{H}$ into $W^{\prime}=\bigoplus_{i=1}^{r} W_{i} / W_{i+1}$ is completely reducible, $\mathbf{H} / \mathbf{U}^{\mathbf{1}}$ is reductive and the connected component $\mathbf{U}$ of identity in $\mathbf{U}^{\mathbf{1}}$ is the unipotent radical of $\mathbf{H}$. We consider the subgroup $H$ (resp. $U$ ) of $\mathbb{F}$-rational points of $\mathbf{H}$ (resp. $\mathbf{U})$, the factor group $X=$ 
$H / U$, the projection $h \mapsto \bar{h}$ of $H$ into $X$, a Borel section $\sigma$ of $X$ into $H$ and we write, for any $h \in H, h=\sigma(\bar{h}) \eta(h)$ with $\eta(h) \in U$. More generally we consider the cocycle $\eta$ from $H \times X$ to $U$ given by

$$
h \sigma(x)=\sigma(\bar{h} x) \eta(h, x) \quad(x \in X) .
$$

We are led to estimate the integrals $\int \log \|\tau(h)\| \mathrm{d} \alpha^{n}(h), \int \log \|\sigma(\bar{h})\| \mathrm{d} \alpha^{n}(h)$ and $\int \log \|\eta(h, e)\| \mathrm{d} \alpha^{n}(h)$. We note that, in view of ([Bo, p. 209), $X=H / U$ is isomorphic to the subgroup $X$ of $\mathbb{F}$-rational points of $\mathbf{H} / \mathbf{U}$.

b) For any $h \in X$, we write

$$
w(\bar{h})=\sup \left\{\|\tau(\bar{h})\|,\left\|\tau(\bar{h})^{-1}\right\|\right\}
$$

and we define

$$
\theta(\bar{h})=\inf _{u \in U} \sup \left\{\|h u\|,\left\|h u^{-1}\right\|\right\} .
$$

Then $\theta$ and $w$ are continuous sub-multiplicative functions on $X$ and we show that, for some constants $c$ and $c^{\prime}$,

$$
\log \theta(\bar{h}) \leq c \log w(\bar{h})+c^{\prime} .
$$

Since $\mathbf{H} / \mathbf{U}$ is reductive, we can write the group $X$ of its $\mathbb{F}$-rational points as $X=$ $\bigcup_{n \geq 0} V^{n}$, where $V$ is a compact neighbourhood of $e$ in $X$ (see [Ma], p. 54). Since the kernel of $\tau$ in $\mathbf{H} / \mathbf{U}$ is finite, as in the proof of Proposition 4.3, we obtain

$$
\delta_{V}(l) \leq A \log w(l)+A^{\prime}, \quad \forall l \in X,
$$

where $A$ and $A^{\prime}$ are constants. Using sub-additivity of $\log \theta$ on $X$ we can write $\log \theta(\bar{h}) \leq B \delta_{V}(\bar{h})$ for some constant $B>0$, hence

$$
\log \theta(\bar{h}) \leq A B \log w(\bar{h})+A^{\prime} B .
$$

c) By the definition of $\theta(\bar{h})$, the section $\sigma$ of $X$ into $H$ can be chosen so that

$$
\sup \left\{\|\sigma(\bar{h})\|,\left\|\sigma(\bar{h})^{-1}\right\|\right\} \leq 2 \theta(\bar{h}) .
$$

Then from b)

$$
\log \sup \left\{\|\sigma(\bar{h})\|,\left\|\sigma(\bar{h})^{-1}\right\|\right\} \leq c \log \sup \left\{\|\tau(\bar{h})\|,\left\|\tau(\bar{h})^{-1}\right\|\right\}+c^{\prime}+\log 2
$$

with the constants $c, c^{\prime}$ from b).

d) We consider the connected component of identity $\mathbf{H}^{\mathbf{0}}$ of $\mathbf{H}$, the finite group $Y=\mathbf{H} / \mathbf{H}^{\mathbf{0}}$ and a section $\beta$ of $Y$ into $\mathbf{H}$. We write for $h \in \mathbf{H}, y \in Y$,

$$
h \beta(y)=\beta(\hat{h} y) \gamma(h, y),
$$

where $\hat{h}$ is the projection of $h$ into $Y$, and $\gamma(h, y) \in \mathbf{H}^{0}$. Since $\tau_{i}$ is irreducible, its restriction to $\mathbf{H}^{\mathbf{0}}$ decomposes as a direct sum of irreducible representations of $\mathbf{H}^{\mathbf{0}}$. We write

$$
\tau_{i}=\bigoplus_{j} \tau_{i j}, \quad W_{i} / W_{i+1}=\bigoplus_{j} W_{i j}
$$

and denote by $d_{i j}$ the dimension of $W_{i j}$. Since $G_{\alpha}$ is amenable, Lemma 4.7 gives the existence of a constant $c_{1}$ such that

$$
\forall h \in G_{\alpha}, y \in Y, \quad\left\|\tau_{i}[h \beta(y)]\right\| \leq c_{1} \sum_{j}\left|\operatorname{det} \tau_{i j}[\gamma(h, y)]\right|^{1 / d_{i j}} .
$$


Hence there exists another constant $c_{2}$ such that

$$
\log \|\tau[h \beta(y)]\| \leq \log c_{1}+c_{2} \sum_{i, j} \log \left|\operatorname{det} \tau_{i j}[\gamma(h, y)]\right| .
$$

We consider the $\mathbb{F}$-character $\chi^{0}$ of $\mathbf{H}^{0}$ defined by $\chi^{0}\left(h_{0}\right)=\Pi_{i j} \operatorname{det} \tau_{i j}\left(h_{0}\right)$ and the cocycle $\bar{\gamma}(h, y)=\chi^{0}[\gamma(h, y)]$. Then the above inequality reads

$$
\log \|\tau[h \beta(y)]\| \leq \log c_{1}+c_{2} \log |\bar{\gamma}(h, y)| .
$$

e) Since $G_{\alpha}$ projects surjectively on $Y$, the normalised counting measure $m_{Y}$ on $Y$ is the unique $\alpha$-stationary measure on $Y$. Then from Birkhoff's ergodic theorem, we get, as in the proof of Proposition 4.6,

$$
\begin{aligned}
\lim _{n} \frac{1}{n} \int \log \|\tau(h)\| \mathrm{d} \alpha^{n}(h) & \leq c_{2} \int \log |\bar{\gamma}(h, y)| \mathrm{d} \alpha(h) \mathrm{d} m_{Y}(y) \\
& =\frac{1}{|Y|} c_{2} \int \log \left|\Pi_{y \in Y} \bar{\gamma}(h, y)\right| \mathrm{d} \alpha(h),
\end{aligned}
$$

where $|Y|$ is the cardinality of $Y$. We note that the cocycle property of $\bar{\gamma}$ implies that $\chi(h)=\prod_{y \in Y} \chi^{0}[\gamma(h, y)]$ is an $\mathbb{F}$-character of $\mathbf{H}$. Then

$$
\lim _{n} \frac{1}{n} \int \log \|\tau(h)\| \mathrm{d} \alpha^{n}(h) \leq \frac{c_{2}}{|Y|} \int \log |\chi(h)| \mathrm{d} \alpha(h) .
$$

Then the hypothesis in the lemma implies

$$
\lim _{n} \frac{1}{n} \int \log \|\tau(h)\| \mathrm{d} \alpha^{n}(h) \leq 0 .
$$

From symmetry of this hypothesis,

$$
\lim _{n} \frac{1}{n} \int \log \|\tau(h)\| \mathrm{d} \alpha^{n}(h)=0 .
$$

Now we can use the subadditive ergodic theorem for the sequence $\log \left\|\tau\left(h_{n} \cdots h_{1}\right)\right\|$; hence we have

$$
\lim _{n} \frac{1}{n} \log \left\|\tau\left(h_{n} \cdots h_{1}\right)\right\|=0
$$

a.e. with respect to the product measure $\alpha^{\otimes \mathbb{N}}$ on $G^{\mathbb{N}}$. It follows that

$$
\lim _{n} \frac{1}{n} \log \sup \left\{\left\|\tau\left(h_{n} \cdots h_{1}\right)\right\|,\left\|\tau\left(h_{1}^{-1} \cdots h_{n}^{-1}\right)\right\|\right\}=0 .
$$

From the inequality stated in c) we conclude that

$$
\lim _{n} \frac{1}{n} \int \log \sup \left\{\|\sigma(\bar{h})\|,\left\|\sigma\left(\bar{h}^{-1}\right)\right\|\right\} \mathrm{d} \alpha^{n}(h)=0 .
$$

f) We observe that $U$ is contained in the subgroup of $(d \times d)$ strictly upper triangular matrices $U_{d}$ with coefficients in $\mathbb{F}$. On $U_{d}$, there are natural subadditive functions comparable to the norm (see [G1] for $\mathbb{F}=\mathbb{R}$ ). Indeed if $u=\left(u_{i j}\right) \in U_{d}$, we define

$$
\begin{aligned}
|u| & =\sup _{1 \leq k \leq d} \sum_{i+j=k+d}\left|u_{i j}\right|^{1 / k} \quad \text { if } \mathbb{F} \text { is connected } \\
& =\sup _{1 \leq k \leq d} \sup _{i+j=k+d}\left|u_{i j}\right|^{1 / k} \quad \text { if } \mathbb{F} \text { is ultrametric }
\end{aligned}
$$


and one can verify that, for any $u, v \in U_{d},|u v| \leq|u|+|v|$. On the other hand, by the definition of $|u|$, we have for some $D>0$,

$$
D^{-1}\|u\|^{1 / d} \leq 1+|u| \leq D\left(1+\|u\|^{d}\right) .
$$

We will use these inequalities in order to estimate $\left|\eta\left(S_{n}\right)\right|$, where $S_{n}$ is the random product $h_{n} \cdots h_{1}$ with respect to the product measure $\alpha^{\otimes \mathbb{N}}$.

g) We use the decompositions of $S_{n}=h_{n} \cdots h_{1}$ introduced in a) and the property stated in $\mathrm{f})$ :

$$
\begin{gathered}
\left\|S_{n}\right\| \leq\left\|\sigma\left(\bar{S}_{n}\right)\right\|\left\|\eta\left(S_{n}\right)\right\|, \\
D^{-1}\left\|\eta\left(S_{n}, e\right)\right\|^{1 / d} \leq\left|\eta\left(S_{n}, e\right)\right| \leq \sum_{i=1}^{n}\left|\eta\left(h_{k}, \bar{b}_{k-1}\right)\right|,
\end{gathered}
$$

where $b_{k}=h_{k-1} \cdots h_{1}$, so that in order to show that $\lim _{n} \frac{1}{n} \log \left\|S_{n}\right\| \leq 0$, it suffices to show that

$$
\varlimsup_{n} \frac{1}{n} \log \left\|\sigma\left(\bar{S}_{n}\right)\right\| \leq 0 \text { and } \lim _{n} \frac{1}{n} \log \left[1+\eta\left(S_{n}\right)\right]=0 .
$$

The first relation has been proved in e). In order to prove the second one, we note that, using the notation in b), for every $h \in H, x \in X$, for $a=4 e^{2 c^{\prime}}$

$$
\|\eta(h, x)\| \leq\|h \sigma(x)\|\left\|\sigma(\bar{h} x)^{-1}\right\| \leq a\|h\| w(\bar{h})^{c} w(x)^{2 c} .
$$

Hence

$$
D^{-1}|\eta(h, x)| \leq\|\eta(h, x)\|^{d} \leq a^{d}\|h\|^{d} w(\bar{h})^{d c} w(x)^{2 d c} .
$$

We fix $\epsilon>0$ and use e) to obtain $w\left(\bar{b}_{k}\right)^{2 d c} \leq e^{k \epsilon}$ with probability 1 , for $k$ large $(k>N)$. Since $\int \log \sup \left\{\|h\|,\left\|h^{-1}\right\|\right\} \mathrm{d} \alpha(h)<\infty$, we have, also with probability 1 , for $k>N$,

Hence we have

$$
\left\|h_{k}\right\| \leq e^{k \epsilon}, \quad w\left(\bar{h}_{k}\right) \leq e^{k \epsilon} .
$$

$$
\begin{aligned}
D^{-1}\left|\eta\left(h_{k}, \bar{b}_{k-1}\right)\right| & \leq a^{d} e^{d \epsilon(1+c) k} e^{2 d c \epsilon k} \\
D^{-1}\left|\eta\left(S_{n}\right)\right| & \leq \sum_{k=1}^{N} \eta\left(h_{k}, \bar{b}_{k-1}\right)+a n e^{d \epsilon(1+3 c) n}
\end{aligned}
$$

where $a>0$ is independent of $n$ and $N<n$. It follows that

$$
\varlimsup_{n} \frac{1}{n} \log \left[1+\left|\eta\left(S_{n}\right)\right|\right] \leq d \epsilon(1+3 c) .
$$

Since $\epsilon$ is arbitrary,

Finally,

$$
\varlimsup_{n} \frac{1}{n} \log \left[1+\left|\eta\left(S_{n}\right)\right|\right]=0 .
$$

$$
\varlimsup_{n} \frac{1}{n} \log \left\|S_{n}\right\| \leq 0 .
$$

From the symmetry of hypothesis, we get

$$
\lim _{n} \frac{1}{n} \log \left\|S_{n}\right\|=0, \text { hence } \lim _{n} \frac{1}{n} \int \log \|g\| \mathrm{d} \alpha^{n}(g)=0 .
$$

Lemma 4.10. Suppose $\alpha \in M^{1, m}(G)$. The condition that $L(\alpha)=0$ implies $G_{\alpha}$ is amenable and $\alpha[\log |f|]=0$ for every algebraic $\mathbb{F}$-character $f$ of $Z^{c}\left(G_{\alpha}\right)$. 
Proof. As in the proof of Proposition 4.3, we have

$$
\delta_{V}(g) \leq d \sum_{i=1}^{r} \log \left\|\rho_{i}(g)\right\|
$$

where $\delta_{V}$ is the sub-additive function considered earlier. Then, Proposition 4.3 implies that, for any $\alpha \in M^{1, m}(G)$,

$$
d \sum_{i=1}^{r} \hat{\omega}_{i}[L(\alpha)] \geq \lim _{n} \frac{\alpha^{n}\left(\delta_{V}\right)}{n} .
$$

From Lemma 3.1 we know that, for some $\gamma>0, e^{-\gamma \delta_{V}} \in L^{2}(G)$. Then, it follows from Lemma 3.3 that

$$
r(\alpha) \geq \lim _{n} \alpha^{n}\left(e^{-\gamma \delta_{V}}\right)^{1 / n} \geq \lim _{n} e^{-\gamma \frac{\alpha^{n}\left(\delta_{V}\right)}{n}} .
$$

Finally,

$$
\gamma d \sum_{i=1}^{r} \hat{\omega}_{i}[L(\alpha)] \geq-\log r(\alpha) .
$$

Hence, the condition $L(\alpha)=0$, implies $r(\alpha)=1$ and hence, the amenability of $G_{\alpha}$ (cf. [DeG]).

We consider a faithful representation $\rho$ of $\mathbf{G}$ in a $\mathbb{F}$ vector space $\mathcal{V}$. Since $\log \|\rho(g)\|$ is sub-additive and continuous, there exists $c>0$ such that with $\delta_{V}$ as above,

$$
\log \|\rho(g)\| \leq c \delta_{V}(g) .
$$

Since $L(\alpha)=0$, we know from the proof above that $\lim _{n} \frac{1}{n} \alpha^{n}\left(\delta_{V}\right)=0$. Hence

$$
\lim _{n} \frac{1}{n} \int \log \|\rho(g)\| \mathrm{d} \alpha^{n}(g)=0 .
$$

If $f$ is an algebraic $\mathbb{F}$-character of $Z^{c}\left(G_{\alpha}\right)$, it is the restriction of a polynomial $p$ on $\mathbf{G}$ to $Z^{c}\left(G_{\alpha}\right)$, and we can write this polynomial $p$ as a polynomial in the coefficients of $\rho(g)$. Hence for some integer $r^{\prime}>0$ : $|p(g)| \leq\|\rho(g)\|^{r^{\prime}}$. Hence $\log |f(g)| \leq r^{\prime} \log \|\rho(g)\|$ for any $g \in Z^{c}\left(G_{\alpha}\right)$. It follows that

$$
\lim _{n} \frac{1}{n} \alpha^{n}(\log |f|)=0 \text {. }
$$

Since $\log |f|$ is additive on $Z^{c}\left(G_{\alpha}\right), \alpha^{n}(\log |f|)=n \alpha(\log |f|)$, hence

$$
\alpha(\log |f|)=\lim _{n} \frac{1}{n} \alpha^{n}(\log |f|)=0 .
$$

Proof of Theorem 1.9. The first three assertions are already stated in Propositions 4.3, 4.6 and Lemma 4.5. If $\alpha, \beta \in F_{\mu}$ and $\mu=\alpha * \beta$, then, with $\delta$ as above, we have $\mu(\delta)<\infty$, hence $\int \delta\left(g g^{\prime}\right) \mathrm{d} \alpha(g) \mathrm{d} \beta\left(g^{\prime}\right)<\infty$ and from Fubini's theorem, there exists $g \in G$ with $\int \delta\left(g g^{\prime}\right) \mathrm{d} \beta\left(g^{\prime}\right)<\infty$. Since

$$
\delta\left(g^{\prime}\right) \leq \delta\left(g^{-1}\right)+\delta\left(g g^{\prime}\right)=\delta(g)+\delta\left(g g^{\prime}\right)
$$

it follows that $\beta(\delta)<\infty$. Hence $L$ is well defined on $F_{\mu}$.

In order to obtain assertion (4), we note that, since $\eta \in \mathcal{S}(\alpha) \subset \mathcal{S}\left(\alpha^{n}\right)$,

$$
H\left(\mu^{n} * \alpha^{n}, \eta\right)=H\left(\mu^{n}, \eta\right)+n H(\alpha, \eta) .
$$


Hence

$$
H(\alpha, \eta)=\lim _{n} \frac{1}{n}\left[H\left((\mu * \alpha)^{n}, \eta\right)-H\left(\mu^{n}, \eta\right)\right] .
$$

Since $Z^{c}\left(G_{\mu * \alpha}\right)=Z^{c}\left(G_{\mu}\right)=G$, the convergence stated in Proposition 4.6 implies that

$$
H(\alpha, \eta)=L(\mu * \alpha)-L(\mu)
$$

Hence $H(\alpha, \eta)$ is constant on $\mathcal{S}(\alpha)$ and Proposition 4.6 implies $H(\alpha, \eta)=L(\alpha)$.

To get the second part we observe that, since $\alpha * \beta=\beta * \alpha$, the theorem of MarkovKakutani implies $\mathcal{S}(\alpha) \cap \mathcal{S}(\beta) \neq \emptyset$, hence we have from above, for $\nu \in \mathcal{S}(\alpha) \cap \mathcal{S}(\beta)$,

$$
L(\alpha)=H(\alpha, \nu), \quad L(\beta)=H(\beta, \nu), \quad L(\alpha * \beta)=H(\alpha * \beta, \nu) .
$$

This implies $L(\alpha * \beta)=L(\alpha)+L(\beta)$.

Now we show the continuity of $L(\alpha)$. Suppose $\left\{\alpha_{n}\right\} \subset F_{\mu}$ converges to $\alpha \in F_{\mu}$. Let $\mu=\alpha_{n} * \alpha_{n}^{\prime}, \alpha_{n}^{\prime} \in F_{\mu},(n \in \mathbb{N})$. It follows that $\left\{\alpha_{n}^{\prime}\right\}$ is relatively compact (cf. P]), hence we can suppose that it has a limit (say) $\alpha^{\prime} \in F_{\mu}$ and $\mu=\alpha * \alpha^{\prime}$. In view of Proposition 4.6, we have

$$
\varlimsup_{n} L\left(\alpha_{n}\right) \prec L(\alpha) \quad \text { and } \quad \varlimsup_{n} L\left(\alpha_{n}^{\prime}\right) \prec L\left(\alpha^{\prime}\right) .
$$

Hence if $\left\{L\left(\alpha_{n}\right)\right\}$ has a subsequence converging to $L(\alpha)-\epsilon$ (where $\epsilon \geq 0$ ), we get, from above,

$$
L(\mu)=L\left(\alpha_{n}\right)+L\left(\alpha_{n}^{\prime}\right) \prec L(\alpha)+L\left(\alpha^{\prime}\right)-\epsilon .
$$

In view of the third assertion, we have $\epsilon=0$, i.e. $\lim _{n} L\left(\alpha_{n}\right)=L(\alpha)$.

Since $H(\alpha, \eta)$ depends continuously on $\alpha \otimes \eta$, assertion (5) follows easily from assertion (4).

One part of the equivalence in assertion (6) follows directly from Lemma 4.10 the converse part follows from Lemma 4.9 and formula (4) in Proposition 4.3

We now explain briefly the proof of assertion (7), since it is an easy extension to the ultrametric situation of known results in the case $\mathbb{F}=\mathbb{R}$ (see GR1], G3]). First, if $\left\{g_{n}\right\}$ is a sequence such that $\lim _{n} g_{n} \cdot m=\delta_{x}$, where $x \in \mathcal{F}$, the use of the decomposition $g_{n}=k_{n} a_{n} k_{n}^{\prime}$ shows that

$$
\forall i \in\{1, \ldots, r\}, \lim _{n} \hat{\alpha}_{i}\left[H\left(g_{n}\right)\right]=\infty,
$$

where $H\left(g_{n}\right) \in \overline{\mathcal{A}^{+}}$is defined in the Introduction. The condition $\lim _{n} \hat{\alpha}_{i}\left[H\left(g_{n}\right)\right]=$ $\infty$ (for all $i$ ) extends the notion of contracting sequence considered in GR1. From the definition of a proximal element in the Introduction we see that if $g$ is proximal, then the sequence $g_{n}=g^{n}$ is contracting. The proof of Theorem 3.1 in GR1] shows that its statement remains valid for a local field $\mathbb{F}$, since the Zariski density implies the total irreducibility used there and the contraction property is satisfied. Hence, we have the following uniform convergence on $\mathcal{F}$ :

$$
\lim _{n} \frac{1}{n} \int H(g, x) \mathrm{d} \alpha^{n}(g)=\int H(g, x) \mathrm{d} \eta(x) \in \mathcal{A}^{+},
$$

where $\eta \in M^{1}(\mathcal{F})$ is the unique $\alpha$-stationary measure on $\mathcal{F}$. Then the property $L(\alpha) \in \mathcal{A}^{+}$follows from assertion (2) in the theorem.

In order to show the converse, we need to recall the representation used in $[\mathrm{Pr}]$ in the case $\mathbb{F}=\mathbb{R}$. We recall that $U$ is the unipotent radical of the minimal parabolic subgroup $P$ associated with $\mathcal{A}^{+}$. Let $\mathcal{G}$ (resp. $\mathcal{U}$ ) be the Lie algebra of $G$ (resp. $U$ ), $p=\operatorname{dim} \mathcal{U}$. Let $\varrho$ be the natural extension to $\bigwedge^{p} \mathcal{G}$ of the adjoint representation of $G$, and let $u$ be a non-zero element in $\bigwedge^{p} \mathcal{U}$. We denote by $W$ the subspace of $\bigwedge^{p} \mathcal{G}$ 
generated by $G \cdot u$. Then the restriction of $\varrho$ to $W$ is irreducible (cf. $[\mathrm{Pr}]$ ), and we will again denote it by $\varrho$. If $\Sigma^{+}$is the system of positive roots defined by $\mathbf{T}$ and $\Pi$, $m_{\lambda}$ is the multiplicity of $\lambda \in \Sigma^{+}$, the highest weight of $\varrho$ is $\tau=\sum_{\lambda \in \Sigma^{+}} m_{\lambda} \lambda$, and $\tau-\alpha_{i}(1 \leq i \leq r)$ are also weights; the other weights are of the form $\tau-\sum_{i=1}^{r} m_{i} \alpha_{i}$ with $m_{i} \geq 0$ and $\sum_{i=1}^{r} m_{i}>0$. We observe that the orbit $G \cdot u$ in $\bigwedge^{p} \mathcal{G}$ is equal to $G / P=\mathcal{F}$, and that the condition of proximality of $g \in G$ is satisfied if $\varrho(g)$ has a simple dominant eigenvalue. We observe that, in that case, $\varrho(g)^{-1}$ also has a simple dominant eigenvalue. As in the proof of Proposition 4.6, we consider the random product $S_{n}(\omega)=g_{n} \cdots g_{1}$, where $\Omega=G^{\mathbb{N}}$ is endowed with the product measure $\pi=\alpha^{\otimes \mathbb{N}}$. For any $d$-dimensional representation $\xi$ of $G$ satisfying

$$
\forall a \in Z_{T}, \quad \xi(a)=\operatorname{diag}\left[\tau_{1}(a), \ldots, \tau_{d}(a)\right],
$$

where the $\tau_{i}$ are $\mathbb{F}$-characters of $\mathbf{Z}_{\mathbf{T}}$, it is easy to show, using the description of Lyapunov exponents given in $\left[\mathrm{R}\right.$ and the polar decomposition of $S_{n}(\omega)$, that the exponents of the product of random matrices $\xi\left(S_{n}\right)$ are the numbers $\hat{\tau}_{i}[L(\alpha)](1 \leq$ $i \leq d)$.

From the description of the weights of $\varrho$, we see that the largest Lyapunov exponent of $\varrho\left(S_{n}\right)$ is $\hat{\tau}[L(\alpha)]$, while the second one is $\hat{\tau}[L(\alpha)]-\inf _{1 \leq i \leq r} \hat{\alpha}_{i}[L(\alpha)]$. Since $L(\alpha) \in \mathcal{A}^{+}$, we get that $\hat{\tau}[L(\alpha)]$ has multiplicity one. Then we can apply Proposition I.3 of [G3], hence there exists a subsequence $\left\{n_{k}(\omega)\right\}$ (of positive density) such that $\varrho\left[S_{n_{k}}(\omega)\right]$ and $\varrho\left[S_{n_{k}}^{-1}(\omega)\right]$ have simple dominant eigenvalues. From above, this implies that for each $k, \varrho\left[S_{n_{k}}(\omega)\right]$ is proximal. Since $S_{n_{k}}(\omega)$ belongs to the semigroup of $G$ generated by $\operatorname{supp} \alpha$, assertion (7) is proved.

Remark. In $[\mathrm{Pr}$, for $\mathbb{F}=\mathbb{R}$, the notion of an $\mathbb{R}$-regular element is used instead of the equivalent notion of proximality. Furthermore, it is shown that the Zariski density of $G_{\alpha}$ implies the existence of proximal elements. This property allows us to recover easily from Theorem 1.9 the simplicity of the Lyapunov spectrum shown in GoMa under the condition of Zariski density of $G_{\alpha}$.

On the other hand, for general $\mathbb{F}$, we can define the notion of $\mathbb{F}$-regular elements as in $[\mathrm{Pr}]: g \in G$ is said to be $\mathbb{F}$-regular if $m(g)$, the number of eigenvalues of Ad $g$ which have modulus 1 is less than $m(h)$ for any $h \in G$. Then we can show that proximality of $g$ is equivalent to $\mathbb{F}$-regularity of $g$ or to $\varrho(g)$ having a simple dominant eigenvalue.

Furthermore, from [Be], we see that if $s_{g^{n}}$ denotes a suitable conjugate of the semisimple part of $g^{n}$ into $\mathbf{Z}_{\mathbf{T}}$ (for some $n$ it belongs to $Z_{T}$ ), then $L\left(\delta_{g}\right)=L(g)=$ $\frac{1}{n} \hat{s}_{g^{n}}$. It follows that proximality of $\mathrm{g}$ is equivalent to $L(g) \in \mathcal{A}^{+}$. Also, we see that if $\alpha=\delta_{g}$, then assertion (6) is the statement of Lemma 4.5 in [Ma] (p. 182).

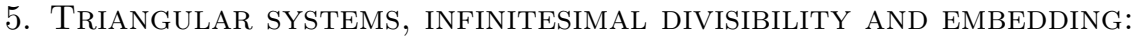 Proofs of Theorems 2.1, 2.3, 1.3}

Proof of Theorem 2.1. Step 1. As in the hypothesis, $S$ is a Hausdorff abelian semigroup with identity $e$ and $s \in S \backslash\{e\}$. Let $\left\{U_{n}\right\}_{n \in \mathbb{N}}$ be a neighbourhood basis of $e$ in $S$ such that $U_{n+1} \subset U_{n}$, for every $n$, and let $F(s)$ and $f_{s}$ be as in the hypothesis such that $e, s \in F(s) \subset F_{s}$ and conditions (1)-(3) are satisfied. Here $f_{s}$ is continuous at $e$ and $f_{s}(s)>0$. Since $s$ is $\left\{U_{n}\right\}$-tree-like divisible in $F(s)$, there exist $U_{n}$-decompositions $s=s_{n 1} \cdots s_{n m_{n}}\left(s_{n i} \in U_{n}\right)$, which are $F(s)$-closed and the $U_{n+1}$-decomposition is finer than the $U_{n}$-decomposition for each $n$. Let $F_{n}=\left\{\prod_{i \in B} s_{n i} \mid B \subset\left\{1, \ldots, m_{n}\right\}\right\}=\bigcup_{\alpha}\left\{\prod_{i=1}^{l} s_{n \alpha(i)} \mid l \leq m_{n}\right\}$, where $\alpha$ is 
any permutation of $\left\{1, \ldots, m_{n}\right\}$. Let $F=\bigcup_{n} F_{n}$. Then it is easy to see that $s \in F \subset F(s)$ and any $a \in F$ is also $\left\{U_{n}\right\}$-tree-like divisible in $F$.

For $\epsilon>0$ such that $\epsilon<1 / 2$, and $a \in F$ such that $f_{s}(a)>0$, let $V_{\epsilon}(a)=\{b \in$ $\left.F(s) \mid f_{s}(b)<\epsilon f_{s}(a)\right\}$, which is a neighbourhood of $e$ in $F(s)$. There exists $n$ such that $U_{n} \cap F(s) \subset V_{\epsilon}(a)$. If $a=s_{n a(1)} s_{n a(2)} \cdots s_{n a(m)}$ is a $U_{n}$-decomposition of $a$ in $F$ as above, then $a=x y$, where $x=s_{n a(1)} s_{n a(2)} \cdots s_{n a(k)} \in F, y=$ $s_{n a(k+1)} \cdots s_{n a(m)} \in F$ and

$$
\frac{1}{2}-\epsilon<\frac{f_{s}(x)}{f_{s}(a)}<\frac{1}{2}
$$

which is obvious if we choose $k$ to be the maximal integer for which

$$
f_{s}\left(s_{n a(1)} \cdots s_{n a(k)}\right)<f_{s}(a) / 2 .
$$

Step 2. Without loss of generality, we may assume $f_{s}(s)=1$ and let $\left\{\epsilon_{j}\right\}$ be a sequence of positive numbers $\prod_{j=1}^{\infty}\left(1-\epsilon_{j}\right)>1 / 2$.

Now we construct a "tree" decomposition of $s$ in $F \subset F(s)$; for indexing we will use sequences $\delta=\left(d_{1}, \ldots, d_{k}\right)$ of 0 and 1 , where $k \in \mathbb{N}$. For $k=0$, we use trivial decomposition $s=t_{\emptyset}$. We take two elements $x$ and $y$ in $F$ according to Step 1 above with

$$
s=x y, \quad \frac{1}{2}-\frac{\epsilon_{1}}{2}<\frac{f_{s}(x)}{f_{s}(s)}<\frac{1}{2},
$$

where $t_{(0)}=x, t_{(1)}=y$. Here $x=s_{n 1} \cdots s_{n k}$, where $n$ is chosen such that $U_{n} \cap$ $F(s) \subset V_{\epsilon_{1} / 2}(s)$, a neighbourhood of $e$ in $F(s)$ defined in Step 1, and $k$ is chosen as in Step 1 such that the above condition is satisfied. Also, $f_{s}(x)<f_{s}(s) / 2=1 / 2$. Let $b(1)=n$. Let $l(\delta)$ denote the length of $\delta$, i.e. for $\delta=\left(d_{1}, \ldots, d_{k}\right), l(\delta)=$ $k$. Suppose $t_{\delta} \in F$ is defined for $\delta$ such that $l(\delta)=k$. Let $r>b(k)$ be such that $U_{r} \cap F(s) \subset \bigcap_{l(\delta)=k} V_{\epsilon_{k} / 2}\left(t_{\delta}\right)$, which is defined in Step 1. Now we take $U_{r^{-}}$ decomposition of $s=s_{r 1} \cdots s_{r m_{r}}$ as in Step 1. We know that $U_{r}$-decomposition of $s$ consists of $U_{r}$-decompositions of all $s_{b(k) i}$ and hence decomposition of $t_{\delta}$, where $l(\delta)=k$. Take $t_{\left(d_{1}, \ldots, d_{k}\right)}=x y$, where $x$ and $y$ in $F$ are chosen as in Step 1 from $U_{r}$-decomposition of $t_{\left(d_{1}, \ldots, d_{k}\right)}$ for $\epsilon=\epsilon_{k}$ and $a=t_{\left(d_{1}, \ldots, d_{k}\right)}$; let $t_{\left(d_{1}, \ldots, d_{k}, 0\right)}=x$, $t_{\left(d_{1}, \ldots, d_{k}, 1\right)}=y$ and $r=b(k+1)$. Thus for all $k$, we can successively define $t_{\delta}$ for $\delta$ of length $k$. Also, for all $k, t_{\delta} \in F_{b(k)}$ if $l(\delta)=k$ and

$$
f_{s}\left(t_{\delta}\right)>2^{-k} \prod_{j=1}^{k}\left(1-\epsilon_{j}\right)>2^{-k-1} .
$$

Step 3. For $d=0$ or 1 , let $x_{k d}$ be the product of all $t_{\delta}$ for the sequence $\delta$ of length $k$ whose $k$ th term is $d$. Then for all $k, x_{k d} \in F_{b(k)}$ for $d=0,1$, and

$$
x_{k 0} x_{k 1}=s \text {. }
$$

For $k \neq m$ and $f, g \in\{0,1\}$ let $y_{k m f g}$ be the product of elements $t_{\delta}$ over the sequences $\delta=\left(d_{1}, \ldots, d_{n}\right)$ of length $n=\max (k, m)$ with $d_{k}=f$ and $d_{m}=g$. Clearly $y_{k m d f}=y_{m k f d}$ and

$$
y_{k m d 0} y_{k m d 1}=y_{m k 0 d} y_{m k 1 d}=x_{k d} \text {. }
$$

Also, $y_{k m d f} \in F_{b(n)}$ and since it is a product of $2^{n-2} t_{\delta}$ 's of length $n$; we have

$$
f_{s}\left(y_{k m d f}\right)>1 / 8 \text {. }
$$


Since $x_{k d} \in F_{b(k)} \subset F(s)$, there exists a sequence $\left\{k_{j}\right\}$ such that

$$
\lim _{j \rightarrow \infty} x_{k_{j} d}=x_{d} \in F(s) \quad(d=0,1) .
$$

Clearly $s=x_{0} x_{1}$.

For every $j$ assign $j^{\prime}>j$ and consider a sequence of points in $(F(s))^{4}$,

$$
z_{j}=\left(y_{k_{j} k_{j^{\prime}} 00}, y_{k_{j} k_{j^{\prime}} 01}, y_{k_{j^{\prime}} k_{j} 10}, y_{k_{j^{\prime}} k_{j} 11}\right),
$$

$z_{j} \in F_{b\left(k_{j^{\prime}}\right)}^{4}$. Passing to a subsequence of $\left\{k_{j}\right\}$ and denoting it by the same notation, we get that $\lim _{n \rightarrow \infty} z_{j}=z \in(F(s))^{4}$, where

$$
z=\left(y_{00}, y_{01}, y_{10}, y_{11}\right) \text {. }
$$

From (5), it follows that

$$
x_{0}=y_{00} y_{01}=y_{00} y_{10} \quad \text { and } \quad x_{1}=y_{10} y_{11}=y_{01} y_{11} .
$$

Multiplying the above two equations, we get that

$$
s=x_{0} x_{1}=y_{00} y_{01}^{2} y_{11} .
$$

Equation (6) and the assumption that $f_{s}$ is continuous at $e$ implies that $y_{01} \neq e$. Also $y_{01} \in \bar{F} \subset F(s)$.

The basic finiteness property of factors is given by the following:

Lemma 5.1. Let $G$ be a locally compact group. Let $\mu \in M^{1}(G)$ be such that $Z_{\mu}^{0} / Z^{0}$ is compact. Let $J_{\mu}=\left\{\lambda \in M^{1}(G) \mid \operatorname{supp} \lambda \subset I(\mu)\right\}$; it is a compact semigroup. Then there exists a neighbourhood $V$ of $J_{\mu}$ such that the following hold:

(1) For any neighbourhood $W$ of $J_{\mu}$ such that $W \subset V$, there exists $n=n(W)$, such that for any $l \geq n, \mu$ cannot be written as $\mu=\mu_{1} * \cdots * \mu_{l}$, where $\mu_{i}$ 's commute and $\mu_{i} \in Z_{\mu}^{0} V \backslash Z_{\mu}^{0} W$.

(2) Moreover, if $\mu$ is the limit of c.i.t.s. $\Delta=\left(\mu_{i j}\right)_{i \in \mathbb{N}, j=1}^{n_{i}},\left(n_{i} \rightarrow \infty\right)$, then for any neighbourhood $U$ of $J_{\mu}$, $\mu$ has a $Z_{\mu}^{0} U$-decomposition; namely, for any neighbourhood $W$ of $J_{\mu}$ such that $W W \subset U$, there exists $n$ such that $\mu=\lambda_{1} \cdots \lambda_{n}$ is a $Z_{\mu}^{0} U$-decomposition, where $\lambda_{1}, \ldots, \lambda_{n-1} \in Z_{\mu}^{0} U \backslash$ $Z_{\mu}^{0} W$ and there exists a sequence $\{k(i)\} \subset \mathbb{N}$ such that, for each $l, \lambda_{l}=$ $\lim _{i \rightarrow \infty} *_{j \in A(l, k(i))} \mu_{k(i) j} z_{k(i)}$ for some sequence $\left\{z_{k(i)}\right\} \subset Z^{0}$, where $A(l, k(i))$ $\subset\left\{1, \ldots, n_{k(i)}\right\},\left\{z_{k(i)}\right\}$ and $\{A(l, k(i))\}$ both depend on $\lambda_{l}$ and, for each $i$, $\left\{1, \ldots, n_{k(i)}\right\}=\bigcup_{l} A(l, k(i))$, a disjoint union.

(3) In particular, if $\mu$ is the limit of a c.i.t.s. with equal components, then for any neighbourhood $U$ of $J_{\mu}$ there exist $n, \lambda$ and $\nu$ such that $\mu=\lambda^{n} \nu$, where $\lambda, \nu \in Z_{\mu}^{0} U$ and they commute.

The proof follows as in the proof of Proposition 2.9 of [Sh3], by replacing $Z$ by $Z^{0}$ there: the proof uses Theorem 2.4 of the same paper which is based on concentration functions on locally compact groups. We observe that the proof of (2) uses the same construction as in $[\mathrm{L}$ (see pp. 168-171), which was also based on concentration functions in $\mathbb{R}^{n}$.

Proposition 5.2. Let $\mu$ be the limit of a c.i.t.s. $\Delta=\left(\mu_{i j}\right)_{i \in \mathbb{N}, j=1}^{m_{i}}\left(m_{i} \rightarrow \infty\right)$, on a locally compact group $G$. Suppose that $Z_{\mu}^{0} / Z^{0}$ is compact. Then there exists a compact subgroup $H \subset I(\mu)$, a closed abelian semigroup $S \subset M_{H}^{1}(G)$ with identity 
$h=\omega_{H}$ such that $Z^{0} h \subset S^{u}, \mu \in S$ and the following hold:

(1) The subgroup $S^{u}$ of units in $S$ is equal to $S \cap Z_{\mu} h$ and $S^{u} / Z^{0} h$ is compact.

(2) There exists a sequence $\{k(i)\} \subset \mathbb{N}$ such that any $\alpha \in S$ is the limit of a c.i.t.s. $\Delta_{\alpha}$ arising from $\Delta$ and elements of $Z^{0} ; \Delta_{\alpha}=\left(\mu_{k(i) j}^{l(i, j)} z_{i}\right)$, where $\left\{z_{i}\right\} \subset Z^{0}$ and $\{l(i, j)\} \subset \mathbb{N} \cup\{0\}$ both depend on $\alpha\left(\lambda^{0}=\delta_{e}\right.$ for all $\left.\lambda \in M^{1}(G)\right)$.

(3) Let $U$ be any neighbourhood of identity $h$ in $S$ and let $\alpha \in F_{\mu}$ (in $S$ ). Then (a) $\alpha$ has a $S^{u} U$-decomposition. (b) In case $\mu_{i j}=\mu_{i}$, i.e. $\mu$ is the limit of a c.i.t.s. with equal components, then there exists $n$ such that $\alpha=\lambda^{n} \nu$ is an $S^{u} U$-decomposition for some $\lambda, \nu \in S$.

(4) $F_{\pi(\mu)}$ is compact in $\pi(S)$ and it is idempotent-free.

(5) $S^{u} \subset Z_{\alpha}^{0} h$ for all $\alpha \in S$.

Proof. The assertions (1)-(4) follow easily as in the proof of Proposition 2.9 of [Sh3]. We give the ideas below and sketch part of the construction of $S$ here for the sake of understanding the underlying technique which we need to prove (5).

Let $J_{\mu}$ be the compact semigroup defined as in Lemma 5.1. Since $\mu$ is the limit of a c.i.t.s. and $Z_{\mu}^{0} / Z^{0}$ is compact, by Lemma 5.1, given any neighbourhood $U$ of $J_{\mu}, \mu$ has a $Z_{\mu}^{0} U$-decomposition and hence $\pi(\mu)$ has a $\pi\left(Z_{\mu}^{0} U\right)$-decomposition.

Let $\left\{U_{n}\right\}$ be a neighbourhood basis of $J_{\mu}, U_{n+1} \subset U_{n}, U_{1} \subset\left\{\lambda \in M^{1}(G) \mid\right.$ $\left.\lambda\left(V^{\prime} I(\mu)\right)>1 / 2\right\}$, where $V^{\prime}$ is a relatively compact neighbourhood of $e$ such that $V^{\prime} I(\mu)$ is contained in an almost connected subgroup of $G$. Then for every $n$, we can choose a $\pi\left(Z_{\mu}^{0} U_{n}\right)$-decomposition of $\pi(\mu)=s_{n 1} \cdots s_{n j_{n}}$ successively, i.e. $\pi\left(Z_{\mu}^{0} U_{n+1}\right)$-decomposition of $\pi(\mu)$ is finer than $\pi\left(Z_{\mu}^{0} U_{n}\right)$-decomposition of $\pi(\mu)$, and each $s_{n l}=\lim _{i \rightarrow \infty} *_{j \in A_{i}} \pi\left(\mu_{k(n, i) j}\right)$, where $A_{i}=A(k(n, i), n, l)$ depend on $n, i, l$, also $\left\{1, \ldots, m_{k(n, i)}\right\}=\bigcup_{l} A(k(n, i), n, l)$ (which is a disjoint union), and $\{k(n+1, i)\}$ is a subsequence of $\{k(n, i)\}$. Hence by a diagonalisation procedure we can choose one sequence $\{k(i)\}$ such that $s_{n l}=\lim _{i \rightarrow \infty} *_{j \in B_{i}} \pi\left(\mu_{k(i) j}\right)$, where $B_{i}=$ $A(k(i), n, l) \subset\left\{1, \ldots, m_{k(i)}\right\}$ and $m_{k(i)}$ is as above. Also, for each $i, A(k(i), n, l)$ depend on $n$ and $l$, and for every $i,\left\{1, \ldots, m_{k(i)}\right\}=\bigcup_{l} A(k(i), n, l)$, a disjoint union.

Now let $X=\bigcup_{n}\left\{s_{n 1}, \ldots, s_{n j_{n}}\right\}, s_{n l}$ defined as above. Let $S(1)_{\pi}$ be the closed semigroup generated by $X$ in $\pi\left(M^{1}(G)\right)$ and let $S(1)=\pi^{-1}\left(S(1)_{\pi}\right)$. Clearly, $S(1)$ is generated by $\pi^{-1}(X)$ as a closed semigroup. Since any element $s \in \pi^{-1}(X)$ is a limit of $\left\{*_{j \in C_{i}} \mu_{k(i) j} z_{i}\right\}, C_{i}=A(k(i), s), S(1)$ is a commutative semigroup and $Z^{0} S(1)=S(1)$. Therefore, since any element of $Z^{0}$ is infinitesimally divisible in it, any element $s \in S(1)$ is the limit of a c.i.t.s. $\left(\mu_{k(i) j}^{l(i, j)} z_{i}\right)$, where $\left\{z_{i}\right\} \subset Z^{0}$, $\{l(i, j)\} \subset \mathbb{N} \cup\{0\}$, they depend on $s$ and $\lambda^{0}=\delta_{e}$ for all $\lambda \in M^{1}(G)$.

Let $J=J_{\mu} \cap S(1)$ and $J_{1}=Z_{\mu}^{0} J_{\mu} \cap S\left(1\right.$ ). Then $J_{1}$ (resp. $J$ ) is a non-empty closed (resp. compact) abelian semigroup. From above, it follows that $\mu$ has a $J_{1} U$-decomposition for any neighbourhood $U$ of $J$ in $S(1)$. Now let $h=\omega_{H}$ be a maximal idempotent in $J$ (it exists because $J$ is compact). Then $H \subset I(\mu)$ is a compact subgroup and $J h$ is a compact abelian group with identity $h($ see $[\mathrm{H}]$, p. 88). Now since $\pi\left(J_{1}\right)=\pi\left[Z_{\mu}^{0} J_{\mu} \cap S(1)\right]$ is a compact semigroup, it has a maximal idempotent, and since it is contained in $\pi(J)$, it is same as $\pi(h)$. Therefore, $J_{1} h$ is also an abelian group with identity $h$. Let $S=S(1) h$ and $S_{\pi}=S(1)_{\pi} \pi(h)$, which are abelian semigroups with identity $h$ and $\pi(h)$ respectively, $Z^{0} h \subset S^{u}, S \subset S(1)$, $S_{\pi} \subset S(1)_{\pi}$ and $S_{\pi}=S / Z^{0}, \mu \in S$. If $h_{1} \in F_{\mu}$ (in $S$ ) is an idempotent, then 
$h_{1} \mu=\mu$ and hence $h_{1} \in J_{\mu} \cap S=J h$. Since $h$ is the maximal idempotent in $J$, $h_{1}=h_{1} h=h$, therefore $F_{\mu}$ (in $S$ ) is idempotent-free and hence so is $F_{\pi(\mu)}$. Also, $Z^{0} h \subset S^{u}=J_{1} h \subset Z_{\mu}^{0} J_{\mu} h \cap S$, so $S^{u} / Z^{0} h$ is compact.

Let $U \subset U_{1}$ be a neighbourhood of $h$ in $S$. Then $U_{n} h \cap S \subset U$ for all large $n$. Since $\pi(\mu)=\prod_{l} s_{n l}=\prod_{l} s_{n l} \pi(h)$ is a decomposition in $\pi\left(J_{1} U_{n} h\right)$ as above, we have $\mu=*_{l} s_{n l}^{\prime} x, s_{n l}^{\prime} \in S \cap U_{n} h, \pi\left(s_{n l}^{\prime}\right)=s_{n l}$ and $x \in J_{1} h=S^{u}$. That is, $\mu$ and also any $s_{n l}$ have a $S^{u} U$-decomposition in $S$. Now for any $\alpha \in F_{\mu}, \alpha=\lim _{n} \alpha_{n} x_{n}$, where $x_{n} \in S^{u}, \alpha_{n}$ are products of $s_{n l}^{\prime}$ which have $S^{u} U$-decomposition, using the facts that $F_{\alpha} \subset F_{\mu}, \alpha_{n}$ 's have $S^{u} U$-decompositons and (1) of Lemma 5.1 one can show that $\alpha$ has $S^{u} U$-decomposition.

Statements (1)-(3) are proven above. From (2) we have that any $\pi(\alpha)$ in $F_{\pi(\mu)}$ has $\pi\left(S^{u} U\right)$-decomposition in $S_{\pi}$, where $U \subset U_{1}$ as above. Also, as $S^{u} / Z^{0} h$ is compact, from the choice of $U_{1}$, we get that $F_{\pi(\mu)} \cap \pi(U)$ is relatively compact. Using these facts and the boundedness of number of factors of elements in $F_{\pi(\mu)}$ as in (1) of Lemma 5.1, statement (4) can be proven as in the proof of Proposition 2.8 in [Sh3], (also see (2)-(3) of Lemma 5.1).

Since $S$ is commutative, for any $\alpha \in S$ and $u \in S^{u}, \alpha=u \alpha u^{-1}=g \alpha g^{-1}$, where $u=\delta_{g} h$, and hence $S^{u} \subset Z_{\alpha} h$.

Now we show that $S^{u} \subset Z_{\alpha}^{0} h$ for all $\alpha \in S$. If possible, suppose there exists $\alpha \in S$ such that $S^{u} \not \subset Z_{\alpha}^{0} h$, i.e. there exists $x \in S^{u} \backslash Z_{\alpha}^{0} h$. Then $x \in L h \backslash L^{0} h$, where $L=Z_{\alpha} \cap Z_{\mu}$. Here $L^{0}=Z_{\alpha}^{0} \cap Z_{\mu}^{0}$ and $H \subset L$. There exists a compact subgroup $K \subset L$ which is $L^{0} H$-invariant and $L^{0} K=K L^{0}$ is an open subgroup of $L$ such that $x \notin L^{0} K h$. Let $U=\left\{\gamma \in M^{1}(G) \mid \gamma(K H W)>1 / 2\right\}$ and $V=\left\{\gamma \in M^{1}(G) \mid\right.$ $\left.\gamma\left(K H W^{\prime}\right)>1 / 4\right\}$ for some relatively compact $K H$-invariant neighbourhoods $W$ and $W^{\prime}$ of $e$ in $G$ such that $W W \subset W^{\prime}, \overline{W^{\prime}} \cap L \subset L^{0} K$. Here, $U U \subset V, K H U=U$, $K H V=V$ and $\bar{V} h \cap L h \subset L^{0} K h$. Moreover, by the choice of $V, \overline{L^{0} V}=L^{0} \bar{V}$ (cf. Sh3], Lemma 2.1). Here, $x$ is the limit of a c.i.t.s. (say), $\left(\mu_{k(i) j}^{l(i, j)} z_{i}\right)$, where $\left\{z_{i}\right\} \subset Z^{0}$ depends on $x$. For simplicity, we rename this c.i.t.s. as $\Delta_{x}=\left(\lambda_{i j} z_{i}\right)_{i \in \mathbb{N}, j=1}^{b_{i}}\left\{z_{i}\right\}$ which converges to $x$. As $U$ is a neighbourhood of $\delta_{e}$, without loss of generality we may assume that each $\lambda_{i j} \in U$. Now for every $i$, if possible, let $c_{i}>1$ be such that

$$
*_{j=1}^{c_{i}-1} \lambda_{i j} \in L^{0} U, \quad \lambda_{i}=*_{j=1}^{c_{i}} \lambda_{i j} \notin L^{0} U
$$

Here, $c_{i}$ exists for all large $i$, because if $*_{j=1}^{b_{i}} \lambda_{i j} \in L^{0} U$ for infinitely many $i$, then since $Z^{0} \subset L^{0}, x \in \overline{L^{0} U} h \cap L h \subset L^{0} K h$, which contradicts our assumption. Here,

$$
\lambda_{i} \in L^{0} U U \backslash L^{0} U \text { for all large } i .
$$

Now we get that $\left\{l_{i} \lambda_{i}\right\} \subset U U \backslash U$ for some $\left\{l_{i}\right\} \subset L^{0}$ for all large $i$. Since $U U \subset V$, by Lemma 2.1 of [Sh3], $\left\{l_{i} \lambda_{i}\right\}$ is relatively compact. Also, since $L^{0} / Z^{0}$ is compact, passing to a subsequence, we may assume that $\left\{z_{i}^{\prime} \lambda_{i}\right\}$ converges to (say) $\beta$ for some $\left\{z_{i}^{\prime}\right\} \subset Z^{0}$. Since $Z^{0} \subset L^{0}$, we get from above that

$$
\forall i, \quad z_{i}^{\prime} \lambda_{i} \in L^{0} U U \backslash L^{0} U \text { and hence } \beta \in \overline{L^{0} U U} \backslash L^{0} U \subset L^{0} \bar{V} \backslash L^{0} U .
$$

From the choice of the system $\Delta_{x}$, we know that $\beta$ commutes with all the elements of $S(1)$ and hence of $S$. Also, $\beta \in F_{x}$ (in $M^{1}(G)$ ). Since $x=x h=\delta_{g^{\prime}} \omega_{H}$ for some $g^{\prime} \in G, \beta$ is supported on a coset of $H$ and $\beta_{1}=\beta h=\delta_{g_{1}} h=h \delta_{g_{1}}$ for some $g_{1} \in G$. Also, for any $\gamma \in S, g_{1} \gamma=\beta_{1} \gamma=\gamma \beta_{1}=\gamma g_{1}$, and hence $\gamma=g_{1} \gamma g_{1}^{-1}$, in particular $g_{1} \in Z_{\alpha} \cap Z_{\mu}=L$. Therefore, $\beta h=\delta_{g_{1}} h \in L h \cap L^{0} \bar{V} h \subset L^{0} K h$, hence 
$g_{1} \in L^{0} K H$. Since $\beta=g_{1} \beta^{\prime}$ where $\beta^{\prime}(H)=1, \beta^{\prime} \in U$ and $\beta \in L^{0} K H U=L^{0} U$, we have a contradiction. Therefore, $S^{u} \subset Z_{\alpha}^{0} h$, for all $\alpha \in S$.

Proof of Theorem [2.3. Since the hypothesis of the Proposition 5.2 is satisfied, we get closed abelian semigroups $S$ and $S_{\pi}=S / Z^{0}$ satisfying conditions (1)-(4). By (4) above, we know that $S^{u} \subset Z_{\alpha}^{0} h$ for all $\alpha \in S$, i.e.

$$
S^{u} \subset L_{1}=\bigcap_{\alpha \in S} Z_{\alpha}^{0} h
$$

Here, $L_{1}$ as above is a closed connected group isomorphic to a subgroup of $N(H) / H$, where $N(H)$ is the normaliser of $H$ in $G$. By the definition of $L_{1}, S^{u}$ is central in $L_{1}$. Therefore, since $L_{1}$ is connected and hence Lie projective, there exists a closed connected abelian subgroup $C \subset L_{1}$ such that $S^{u} \subset C \subset L_{1}$ (see Theorem 1.2 in $\mathrm{Ch}$. XVI in [Ho], which also holds for connected groups). Also, since $Z_{\mu}^{0} / Z^{0}$ is compact and $Z^{0} h \subset S^{u} \subset L_{1} \subset Z_{\mu}^{0} h$, we get that $C / Z^{0} h$ is compact. Let $S^{\prime}=C S$ and $S_{\pi}^{\prime}=\pi\left(S^{\prime}\right)=\pi(C) \pi(S)$. Now the set $\left(S^{\prime}\right)^{u}$, the group of units in $S^{\prime}$, is clearly the same as the group $C$ which is a connected abelian group, and it is compact modulo $Z^{0} h$.

Clearly for $\pi$ as above, the statements (1) and (2) and (4) and (5) of Proposition 5.2 imply that the same statements hold for $S^{\prime}$ and $\pi\left(S^{\prime}\right)$. Renaming $S^{\prime}$ by $S$, the assertions (1)-(3) of the theorem hold.

In the case $\mu$ is the limit of a c.i.t.s. with equal components, then for a neighbourhood basis $\left\{U_{n}\right\}$ of $h$ in $S$ we have $\mu=\lambda_{n}^{k_{n}} \nu_{n}$ as a $S^{u} U_{n}$-decomposition of $\mu$ in $S$. Then there exist $u_{n} \in S^{u}$ and $\nu_{n}^{\prime} \in U_{n}$ such that $\nu_{n}=u_{n} \nu_{n}^{\prime}$. Then $\mu=\lambda_{n}^{k_{n}} u_{n} \nu_{n}^{\prime}$. Since $S^{u}$ is connected, $u_{n}$ is divisible and hence there exists $b_{n} \in S^{u}, b_{n}^{k_{n}}=u_{n}$. Replacing $\lambda_{n}$ by $\lambda_{n} b_{n}$, we get that $\mu=\lambda_{n}^{k_{n}} \nu_{n}^{\prime}$. Hence, $\mu=\lim _{n \rightarrow \infty} \lambda_{n}^{k_{n}}$ as $\nu_{n}^{\prime} \rightarrow h$. Clearly, $\lambda_{n} \in S^{u} U_{n}$ and hence $\pi\left(\lambda_{n}\right) \rightarrow \pi(h)$.

In the case of a normal system, it is clear that each element in $S$ is normal since it comes as a limit of a subsystem with a suitable shift which is also normal.

Lemma 5.3. Let $G$ be a locally compact group and let $\mu \in M^{1}(G)$. Suppose $\mu$ is infinitely divisible and suppose for every $n$ there exists an $n$-root $\mu_{n}$ such that the set $A=\left\{\mu_{n}^{m} \mid 1 \leq m \leq n, n \in \mathbb{N}\right\}$ has relatively compact image on $G / Z^{0}$, i.e. $\pi(A)=A / Z^{0}$ is relatively compact. Then $A$ is relatively compact. Moreover, there exist a semigroup $\Sigma=\left\{\mu_{t}\right\}_{t \geq 0} \subset \bar{A}, \mu_{0}=\omega_{H}$, and a compact subgroup $C \subset Z_{\mu}$ such that $\mu$ is quasi-embeddable in $\Sigma$ relative to $C$ and $C \omega_{H} \subset \bar{A}$. Moreover, if $\mathrm{C} / \mathrm{H}$ is arcwise connected, then $\mu$ itself is embeddable.

Proof. Since $A / Z^{0}$ is relatively compact and since $Z^{0}$ is a connected central subgroup, $A$ is relatively compact (see Lemma 3.2 in [Sh1] or the proof of Proposition 8 in $[\mathbf{M}]$ ). Therefore, $\mu$ is rationally embeddable in $\{\psi(r)\}_{r \in \mathbb{Q}} \subset \bar{A}$ as $\psi(1)=\mu$ such that $\psi(] 0,1[\cap \mathbb{Q})$ is relatively compact. Then $K=\bigcap_{r>0} \overline{\psi(] 0, r[\cap \mathbb{Q})}$ is a compact connected abelian group with identity $\omega_{H}$ and there exists $\Sigma=\left\{\mu_{t}\right\}_{t \geq 0} \subset \overline{\psi(\mathbb{Q})} \subset$ $\bar{A}, \mu_{0}=\omega_{H}$, and $k \in K$ such that $k=x \omega_{H}$ and $k \mu=x \mu=\mu_{1}$, i.e. $x \mu=\mu x$ is embeddable (see Theorems 3.1.32, 3.5.1 in [H]). Let $C=\left\{g \in G \mid g \omega_{H} \in K\right\}$. Then $C$ is a compact subgroup of $Z_{\mu}$ and $C / H$ is connected and abelian, also $C \subset \cap Z_{\mu_{t}}$. Now, since $\mu \in C \Sigma, \mu$ is quasi-embeddable in $\Sigma$ with respect to $C$. Also, $C \omega_{H}=K \subset \bar{A}$. Here, $C^{0}$ is compact and connected and $C=C^{0} H$. If $C / H$ is also arcwise connected, then so is $K$. This implies that $k^{-1}=x^{-1} \omega_{H}$ is embeddable, and hence $\mu$ is embeddable. 
Proof of Theorem 1.3. By Theorem 2.3 there exists a closed abelian semigroup $S \subset M_{H}^{1}(G)$ with identity $h=\omega_{H}$, such that $F_{\mu} / Z^{0}$ is compact, the group $S^{u}$ of invertible elements in $S$ is connected and abelian and $\mu=\lim _{n \rightarrow \infty} \lambda_{n}^{k_{n}}$, where $\pi\left(\lambda_{n}\right) \rightarrow \pi(h)$. Let $B=\left\{\lambda_{n}^{m} \mid 1 \leq m \leq k_{n}, n \in \mathbb{N}\right\}$. Then $B$ is relatively compact as $B / Z^{0}$ is so (cf. [Sh1], Lemma 3.2). For every $m, \mu=\lim _{n \rightarrow \infty} \lambda_{n}^{m\left[k_{n} / m\right]} \nu_{n}$ for some $\nu_{n} \in\left\{\lambda_{n}, \ldots, \lambda_{n}^{m-1}\right\}$. Hence $\pi\left(\nu_{n}\right) \rightarrow \pi(h)$. Therefore, passing to a subsequence if necessary, we get that $\mu=\mu_{m}^{m} u_{m}$, where $\mu_{m}, u_{m}$ are respectively limits of $\left\{\lambda_{n}^{\left[k_{n} / m\right]}\right\}$ and $\left\{\nu_{n}\right\}$, and $u_{m} \in S^{u}$. Now since $S^{u}$ is divisible, there exists $b_{m} \in S^{u}$ such that $b_{m}^{m}=u_{m}$. Replacing $\mu_{m}$ by $\mu_{m} b_{m}$, we get that $\mu=\mu_{m}^{m}$ for all $m$, i.e. $\mu$ is infinitely divisible in $F_{\mu}$ in $S$. Since $F_{\mu} / Z^{0}$ is compact, so is $A / Z^{0}$, where $A=\left\{\mu_{n}^{m} \mid \mu_{n}^{n}=\mu, 1 \leq m \leq n, n \in \mathbb{N}\right\}$. Now by Lemma $5.3 A$ is relatively compact and there exists a continuous one-parameter semigroup $\Sigma=\left\{\mu_{t}\right\}_{t \geq 0} \subset \bar{A}, \mu_{0}=\omega_{H}$, and a compact subgroup $C \subset Z_{\mu}$ which commutes with $\Sigma$ and normalises $H$, such that $\mu$ is quasi-embeddable in $\Sigma$ with respect to $C$ and $C \omega_{H} \subset \bar{A}$. Moreover, if every compact connected subgroup of $Z_{\mu}^{0}$ is arcwise connected, so is $C / H=C^{0} H / H$ and hence $\mu$ itself is embeddable.

\section{Construction of Semi-Groups AND $s$-NORMS USING HARMONIC ANALYSIS}

In this section, for a 'full' measure $\mu$ which is the limit of a c.i.t.s. on an amenable subgroup of an algebraic group, we construct an abelian semigroup containing $\mu$ with properties required in Corollary 2.2. In this case, it is easy to find required abelian semigroups (see Theorem 2.3) but $s$-norms will in general exist only on subsets of such semigroups, hence a detailed analysis using finite-dimensional representations is needed. The main point is constructing $F(s)$ and $s$-norm on $F(s)$ satisfying the conditions of Theorem 2.1. We start by proving a basic proposition.

Proposition 6.1. Let $G$ be a locally compact group. Let $S$ be a closed abelian semigroup with identity $\omega_{H}$ in $M_{H}^{1}(G)$. Suppose there exists a continuous homomorphism $\phi: G \rightarrow \mathbf{S}^{1}$, the circle group, such that $\phi\left(S^{u}\right)=\left\{\delta_{e}\right\}$, where $e=1$ is the identity of $\mathbf{S}^{1}$. Suppose $\lambda \in S$ is such that any $\nu \in F_{\lambda}$ is infinitesimally divisible in $S, F_{\bar{\lambda}}$ is compact, associate-free and idempotent-free and $\phi(\lambda)=\delta_{x_{0}}$ for some $x_{0} \neq e$. Then there exists $\alpha \in F_{\lambda} \backslash S^{u}$ and a compact subset $F(\bar{\alpha}) \subset F_{\bar{\alpha}}$ (in $S_{\xi}$ ) such that $\bar{\alpha}$ and $F(\bar{\alpha})$ satisfy conditions (1)-(3) of Theorem 2.1 .

Proof. Since $\phi\left(S^{u}\right)=\left\{\delta_{e}\right\}$, we have $\phi(\beta)=\phi(\beta x)$ for all $\beta \in S$ and $x \in S^{u}$ and hence we can define $\bar{\phi}: S_{\xi} \rightarrow M^{1}\left(\mathbf{S}^{1}\right)$ as $\bar{\phi}(\bar{\beta})=\phi(\beta)$. Here, $\bar{\phi}$ is a continuous homomorphism. We may also identify $x$ and $\delta_{x}$ for any $x \in \mathbf{S}^{1}$. Since $\phi(\lambda)=x_{0} \neq e$, each element of $\phi\left(F_{\lambda}\right)$ is a point in $\mathbf{S}^{1}$. Let $V=\left\{e^{i y} \mid-\pi / 4<y<\pi / 4\right\}$. Then $V$ is a symmetric neighbourhood of $e$ in $\mathbf{S}^{1}$. Let $V^{+}=\left\{e^{i y} \mid 0 \leq y<\pi / 4\right\}$ and $V^{-}=\left(V^{+}\right)^{-1}$. Let $\left\{U_{n}\right\}$ be a neighbourhood basis of $\omega_{H}$ in $S$ such that, for all $n$, $U_{n+1}^{2} \subset U_{n}$ and $\phi\left(U_{n}\right) \subset V$. Then for any $n$,

$$
\lambda=\alpha_{n 1} \cdots \alpha_{n m(n)} \beta_{n 1} \cdots \beta_{n k(n)} \gamma_{n 1} \cdots \gamma_{n l(n)},
$$

where for each $j$,

$$
\phi\left(\alpha_{n j}\right) \in V^{+} \backslash\{e\}, \phi\left(\beta_{n j}\right) \in V^{-} \backslash\{e\} \text { and } \phi\left(\gamma_{n j}\right)=e .
$$

Let

$$
\alpha_{n}=*_{j=1}^{m(n)} \alpha_{n j}, \beta_{n}=*_{j=1}^{k(n)} \beta_{n j} \text { and } \gamma_{n}=*_{j=1}^{l(n)} \gamma_{n j} .
$$


Then since $F_{\bar{\lambda}}$ is compact, passing to a subsequence if necessary, we may assume that

$$
\bar{\alpha}_{n} \rightarrow \overline{\alpha^{\prime}}, \bar{\beta}_{n} \rightarrow \overline{\beta^{\prime}}, \bar{\gamma}_{n} \rightarrow \overline{\gamma^{\prime}}
$$

and

$$
\lambda=\alpha^{\prime} \beta^{\prime} \gamma^{\prime}, \quad \alpha^{\prime}, \beta^{\prime}, \gamma^{\prime} \in S
$$

Since $\phi(\lambda)=x_{0} \neq e$ and $\phi\left(\gamma^{\prime}\right)=e$, either $\phi\left(\alpha^{\prime}\right)$ or $\phi\left(\beta^{\prime}\right)$ is non-trivial. Hence with possible interchange of $\phi\left(\alpha^{\prime}\right)$ and $\phi\left(\beta^{\prime}\right)^{-1}$ we can suppose $\phi\left(\alpha^{\prime}\right)=a^{\prime} \neq e$. We now choose a sequence $\left\{m^{\prime}(n)\right\}$, where $m^{\prime}(n) \leq m(n)$ for all $n$, as follows: Let

$$
U=\left\{g=e^{i y} \mid-\min \left\{\pi / 9,-i\left(\log a^{\prime}\right) / 2\right\}<y<\min \left\{\pi / 9,-i\left(\log a^{\prime}\right) / 2\right\}\right\} .
$$

$\phi\left(U_{n}\right) \subset U$ for all large $n$ and $\phi\left(*_{j=1}^{i} \alpha_{n j}\right) \in U$ for all $i<m^{\prime}(n)$ but $\phi\left(*_{j=1}^{m^{\prime}(n)} \alpha_{n j}\right) \notin$ $U$. Here, $\overline{U U} \subset V$. Now again passing to a subsequence if necessary, we get that $*_{j=1}^{m^{\prime}(n)} \bar{\alpha}_{n j}$ converges to $\bar{\alpha}$, for some $\alpha \in F_{\lambda}$; i.e. $\bar{\alpha}$ is the limit of a c.i.t.s. $\Delta_{\bar{\alpha}}=\left(\bar{\alpha}_{n j}\right)_{n \in \mathbb{N}, j=1}^{m^{\prime}(n)}$ in $S_{\xi}$, and hence $\phi(\alpha)=a \in V^{+} \backslash U$.

Since $F_{\bar{\alpha}} \subset F_{\bar{\lambda}}$ is compact in $S_{\xi}$, as in the proof of Lemma 2.6 of [Sh3] or using Lemma [5.1] above, we can show that $\bar{\alpha}$ has a $U_{n}^{\prime}$-decomposition obtained as a limit of the subsystem $\Delta_{\bar{\alpha}}$ in $S_{\xi}$, where $U_{n}^{\prime}$ is the image of $U_{n}$ in $S_{\xi}$. As in the proof of Proposition 5.2 above, we get a $U_{n}^{\prime}$-decomposition of $\bar{\alpha}$, which is obtained successively from the system $\Delta_{\bar{\alpha}}$ in $S_{\xi}$, i.e.

$$
\bar{\alpha}=s_{n 1}^{\prime} \cdots s_{n r(n)}^{\prime},
$$

where $s_{n 1}^{\prime}, \ldots, s_{n r(n)}^{\prime}$ are also limits of the partial products of a same subsystem of $\Delta_{\bar{\alpha}}$ and for every $n$, the $U_{n+1}^{\prime}$-decomposition is finer than the $U_{n}^{\prime}$-decomposition. Let us fix any $n \in \mathbb{N}$. Let

$$
F_{n}=\left\{*_{j \in D} s_{n j}^{\prime} \mid D \subset\{1, \ldots, r(n)\}\right\} .
$$

Now, it is easy to see that for each $n, F_{n} \subset F_{n+1}$ and $\bar{\phi}(\bar{\nu}) \in V^{+}$, for all $\bar{\nu} \in F_{n}$. Hence defining $F(\bar{\alpha})=\overline{\bigcup_{n} F_{n}}$, we have $\bar{\phi}[F(\bar{\alpha})] \subset \overline{V^{+}}, \bar{\alpha} \in F(\bar{\alpha}) \subset F_{\bar{\alpha}} \subset F_{\bar{\lambda}}$, $\overline{\omega_{H}} \in F(\bar{\alpha})$. Since $\bar{\lambda}, F_{\bar{\lambda}}$ satisfy condition (1) of Theorem 2.1, the same is true for $\bar{\alpha}, F_{\bar{\alpha}}$.

Furthermore, from the above construction, $\bar{\alpha}$ is $U_{n}^{\prime}$-tree-like divisible in $F(\bar{\alpha})$. Also $\bar{\phi}[F(\bar{\alpha})] \subset \overline{V^{+}}$, hence we can define a continuous partial homomorphism $f_{\bar{\alpha}}$ on $F(\bar{\alpha})$ to $[0, \pi / 4] \subset[0,1]$ as $f_{\bar{\alpha}}(\bar{\nu})=-i \log [\bar{\phi}(\bar{\nu})]=-i \log [\phi(\nu)]$ for every $\nu$ such that $\bar{\nu} \in F(\bar{\alpha})$. Here $f_{\bar{\alpha}}(\bar{\alpha})=-i \log a>0$ as $a \in V^{+} \backslash\{e\}$. Hence condition (3) of Theorem 2.1 is satisfied.

Remarks. 1. Later on, we will encounter homomorphisms from $G$ to a locally compact abelian group $G^{\prime}$, but composing them with a non-trivial character from $G^{\prime}$ to $\mathbf{S}^{1}$, we can apply the above proposition.

2. The above proposition is also valid if we have a continuous homomorphism from the semigroup $S$ as above to $\mathbf{S}^{1}$ or even a partial homomorphism from $F_{\lambda}$ (in $S)$ to $\mathbf{S}^{1}$, along with the rest of the hypothesis.

The following can be derived easily using the above result and Proposition 2.8 of [Sh3] or Theorem 2.3. It shows in particular the essential role of the spectral properties in the problem. 
Proposition 6.2. Let $\mu$ be the limit of a c.i.t.s. on a discrete group G. Then $\mu=\lambda * \nu=\nu * \lambda$, where $\lambda$ is embeddable in a continuous one-parameter semigroup $\left\{\lambda_{t}\right\}_{t \geq 0}, \lambda_{0}=\omega_{H}$, and $\nu \in S_{1}$, where $S_{1}$ is a closed abelian semigroup with identity $\omega_{H}$ with the following properties:

(1) $F_{\nu}$ (in $S_{1}$ ) is compact, associate-free and idempotent-free.

(2) Every $\gamma \in S_{1}$ commutes with $\lambda_{t}$, for each $t$.

(3) Every $\gamma \in F_{\nu}\left(\right.$ in $\left.S_{1}\right)$ is infinitesimally divisible.

(4) For every $\gamma \in S_{1}$, the spectrum of the convolution operator $\rho(\gamma)$ on $L^{2}(G)$ is contained in $\{0,1\}$ and $\rho(\gamma)$ is invertible in the commutative algebra generated by $\rho\left(S_{1}\right)$ with identity $\rho\left(\omega_{H}\right)$.

Remark. The above in particular implies that the limit $\mu$ of a c.i.t.s. on a discrete group $G$ is embeddable if any $\omega_{H}$-infinitesimally divisible element $\nu \in F_{\mu}$, with the spectrum of $\rho(\nu)$ contained in $\{0,1\}$, is an idempotent. This, in particular, holds and is easy to see if $G_{\nu} \subset G$ is contained in a group which is a compact extension of a closed solvable normal subgroup. This property also holds for any countable linear group $G$ over a local field $\mathbb{F}$. (We do not know any counterexample to the above property.) In particular, using Lemma 4.8 and DeG], we obtain an extended form of Theorem 3.9(3) of [Sh3] below.

Corollary 6.3. Suppose $\Gamma$ is a countable subgroup of an algebraic group $G$ defined over a local field $F$, and $\mu \in M^{1}(\Gamma)$ is the limit of a c.i.t.s. on $\Gamma$. Then $\mu$ is embeddable.

Proposition 6.4. Let $G$ be a locally compact group. Let $S$ be a closed abelian semigroup with identity $h=\omega_{H}$ in $M_{H}^{1}(G)$ such that $Z^{0} h \subset S^{u}$ and $S^{u} / Z^{0} h$ is compact. Let $\mu \in S \backslash S^{u}$ be such that $\operatorname{supp} \mu$ is contained in a closed subgroup $L_{\mu}$, which is a compact extension of a closed solvable normal subgroup of itself. Let $\lambda \in F_{\mu} \backslash S^{u}$ be such that any $\nu \in F_{\lambda}$ is infinitesimally divisible in $S$ and $F_{\bar{\lambda}}$ is compact, associate-free and idempotent-free. Then there exists $\alpha \in F_{\lambda} \backslash S^{u}$ and a compact subset $F(\bar{\alpha}) \subset F_{\bar{\alpha}}$ in $S_{\xi}$ such that $\bar{\alpha}$ and $F(\bar{\alpha})$ satisfy conditions (1)-(3) of Theorem 2.1.

Proof. Step 1: Since $S^{u} \subset F_{\lambda}$, any element in $S^{u}$ is infinitesimally divisible and hence $S^{u}$ is connected. Let $C=\bigcup_{\nu \in S^{u}} \operatorname{supp} \nu ; C$ is a closed subgroup and $C / H$ is isomorphic to $S^{u}$. Let $G(\lambda)$ be the closed subgroup generated by all $\operatorname{supp} \nu, \nu \in F_{\lambda}$. Then $C \subset G(\lambda)$. Let $N_{\lambda}$ be the smallest closed normal subgroup in $G_{\lambda}$ such that $\operatorname{supp} \lambda \subset x N_{\lambda}$, for any $x \in \operatorname{supp} \lambda$.

Let $\nu \in F_{\lambda}$. It is easy to show that $\operatorname{supp} \nu \subset y N_{\lambda}=N_{\lambda} y$ for any $y \in \operatorname{supp} \nu$. That is, $N_{\lambda}$ is normal in $G(\lambda)$. Since $S$ is abelian, so is $G(\lambda) / N_{\lambda}$ and hence, $G / \overline{C N_{\lambda}}$ is also abelian. Let $\phi: G(\lambda) \rightarrow G(\lambda) / \overline{C N_{\lambda}}$ be the natural map. Then $\phi(\nu)=\delta_{\phi(y)}$ for any $y \in \operatorname{supp} \nu$. Since $\nu$ is infinitesimally divisible, so is $\phi(y)$, and hence $\phi(y)$ belongs to the connected component of the identity in $G(\lambda) / \overline{C N_{\lambda}}$. Hence, since $G(\lambda) / \overline{C N_{\lambda}}$ is generated by $\left\{\phi(y) \mid y \in \operatorname{supp} \nu, \nu \in F_{\lambda}\right\}, G(\lambda) / \overline{C N_{\lambda}}$ is connected. Now suppose $G(\lambda) \neq \overline{C N_{\lambda}}$. That is, there exists a $\beta \in F_{\lambda}$ such that $\phi(\beta)=\delta_{z}$, $z \neq e$. Also, $\phi\left(S^{u}\right)=\left\{\delta_{e}\right\}$. Now if we take $S_{\lambda}$ to be the closed semigroup generated by $F_{\lambda}$ in $S$, then $S_{\lambda} \subset M^{1}(G(\lambda))$ and $S_{\lambda}^{u}=S^{u}$, hence by Proposition 6.1 and Remark 1 following it, we can construct $\alpha \in F_{\lambda}$ and $F(\bar{\alpha}) \subset F_{\lambda}$ as desired.

Step 2: Now suppose $G(\lambda)=\overline{C N_{\lambda}}$. Since $C / Z^{0}$ is relatively compact, we have $C=K \times V$, where $V \subset Z^{0}$ is a vector group, $K$ is a compact group and $H$ as above 
is contained in $K \cap N_{\lambda}$. Therefore, $G(\lambda)=K \overline{V N_{\lambda}}=K N^{\prime}$, where $N^{\prime}=\overline{V N_{\lambda}}$ is a closed normal subgroup of $G(\lambda)$.

Since $\lambda \in F_{\mu}$, supp $\nu \subset x G_{\mu}=G_{\mu} x$, for all $\nu \in F_{\lambda}$, hence $N_{\lambda} \subset G_{\mu}$. Therefore, $N_{\lambda} \subset L_{\mu}$ which is a compact extension of a closed solvable normal subgroup $R_{\mu}$ (say). Since $V$ is central, this implies that $\overline{V R_{\mu}}$ is solvable and $\overline{V L_{\mu}} / \overline{V R_{\mu}}$ is compact. Now, without loss of generality we may assume that $S$ is generated by $F_{\lambda}$.

For any $\nu \in F_{\lambda}, \nu=k \nu^{\prime}, \operatorname{supp} \nu^{\prime} \subset N^{\prime}$. Clearly, $\nu^{\prime} \in F_{\lambda}$. Also, since $H \subset N^{\prime}$, for $\nu$ as above, $\nu=\nu_{1} * \cdots * \nu_{n}$ in $S$, then $\nu^{\prime}=\nu_{1}^{\prime} * \cdots * \nu_{n}^{\prime}$ where for each $i$, $\nu_{i}=k_{i} \nu_{i}^{\prime}, k_{i} \in K$ and $\nu_{i}^{\prime}$ is supported on $N^{\prime}$. This implies that for each $\nu \in F_{\lambda}, \nu^{\prime}$ is infinitesimally divisible in $S \cap M_{H}^{1}\left(N^{\prime}\right)$. Let $\lambda=k^{\prime} \lambda^{\prime}, k^{\prime} \in K$ and $\lambda^{\prime} \in F_{\lambda} \cap M_{H}^{1}\left(N^{\prime}\right)$ and let $S^{\prime}=\left\{\nu^{\prime} \in S \cap M_{H}^{1}\left(N^{\prime}\right) \mid \nu \in S, \nu=k^{\prime} \nu^{\prime}\right.$ for some $\left.k^{\prime} \in K\right\}$. Then $\lambda^{\prime} \in S^{\prime} \subset S$ and each element in $F_{\lambda^{\prime}}$ (in $S^{\prime}$ ) is infinitesimally divisible in $S^{\prime}$. Since $N_{\lambda}=N_{\lambda^{\prime}}$, without loss of generality we may assume that $\lambda=\lambda^{\prime}$ and $S=S^{\prime}$ and hence $G(\lambda)=N^{\prime}=\overline{V N_{\lambda}}$. Since $S \subset M_{H}^{1}(G(\lambda))$ and $G(\lambda)=\overline{V N_{\lambda}} \subset \overline{V L_{\mu}}$, without loss of generality we may assume that $G=\overline{V L_{\mu}}$ which is a compact extension of the closed solvable normal subgroup $\overline{V R_{\mu}}$.

Step 3: Let $\pi_{1}: G \rightarrow G / G^{0}$ be the natural projection and suppose that $\pi_{1}(\lambda)$ is not a translate of an idempotent. Then since it is infinitesimally divisible in $S_{1}=\overline{\pi_{1}(S)}$, there exists a $\pi_{1}(\lambda)$-norm on $F_{\pi_{1}(\lambda)}$ (in $S_{1}$ ) (cf. Sh3], Theorem 3.6), hence we can define a $\lambda$-norm $f_{\lambda}$ on $F_{\lambda}$. Since $f_{\lambda}\left(S^{u}\right)=\{0\}, f_{\bar{\lambda}}=f_{\lambda} \circ \xi^{-1}$ is well defined and is a $\bar{\lambda}$-norm on $F(\bar{\lambda})=F_{\bar{\lambda}}$ in $S_{\xi}$, and the assertion holds for $\alpha=\lambda$. Now let $\pi_{1}(\lambda)$ be a translate of an idempotent. Then $\pi_{1}(\lambda)=\pi_{1}\left(\omega_{H}\right)$ as $\lambda$ is infinitesimally divisible in $S$ (cf. [Sh3], Lemma 3.2). Therefore, since $V \subset G^{0}$, we have $G(\lambda)=\overline{V N_{\lambda}} \subset H G^{0}$. Also, since $H G^{0}$ is an amenable almost connected group, $H G^{0} / R$ is compact, where $R$ is the maximal connected solvable normal subgroup of $G$. Therefore, we may assume that $G=H G^{0}$.

Step 4: Let $\pi_{2}: G \rightarrow G / R$ be the natural projection. Suppose $\pi_{2}(\lambda)$ is not a translate of an idempotent. Then since $G / R$ is compact, by Lemma 3.3 of [Sh3] there exists a $\pi_{2}(\lambda)$-norm on $F_{\pi_{2}(\lambda)}$ in $S_{2}=\overline{\pi_{2}(S)}$, and the assertion follows as in Step 3. Now let $\pi_{2}(\lambda)$ be a translate of and idempotent. Then by Lemma 3.1 of [Sh3], $\pi_{2}(\lambda)=\delta_{x} * \omega_{\pi_{2}(H)}=\omega_{\pi_{2}(H)} * \delta_{x}$, for some $x$, and hence $N_{\lambda} \subset H R$. Now since $G(\lambda)=N^{\prime}=\overline{V N_{\lambda}}$ and $V \subset R$, we get that $G(\lambda) \subset H R$. Therefore, $\pi_{2}(\lambda)=\omega_{\pi_{2}(H)}=\pi_{2}(\nu)$ for any $\nu \in F_{\lambda}$. Hence we may assume that $G=H R$.

Step 5: We now prove that there exists a $\lambda$-norm on $F_{\lambda}$ in $S$. Then $\alpha=\lambda$ and $F(\bar{\alpha})=F_{\bar{\lambda}}$ will satisfy conditions of Theorem 2.1. We prove this by induction on the length $n$ of $R$, where $G=H R$, i.e. the smallest $n$, such that $R_{n}=\{e\}$, where $R_{1}=\overline{[R, R]}$ and $R_{k+1}=\overline{\left[R_{k}, R_{k}\right]}, k>1$. Suppose $n=1$, i.e. $R$ is abelian. Then, as $\lambda$ is not a translate of an idempotent, the result follows exactly as in Step 4 of the proof of Theorem 3.4 of [Sh3], i.e. there exists a $\lambda$-norm on $F_{\lambda}$ in $S$. Now suppose the statement holds for a group $G=H R$ with length of $R$ less than $n$. Now assume that the length of $R$ is $n$. Let $L=\overline{V R_{1}}$ and let $\pi_{3}: G \rightarrow G(1)=G / L$ be the natural projection, where $R^{\prime}=R / L$ is abelian. If $\pi_{3}(\lambda)$ is not a translate of an idempotent, then as above, there exists a $\pi_{3}(\lambda)$-norm $f$ on $F_{\pi_{3}(\lambda)}$ in $S_{3}=\overline{\pi_{3}(S)}$, and we can define a $\lambda$-norm on $F_{\lambda}$ as $f_{\lambda}=f \circ \pi_{3}$. Now suppose $\pi_{3}(\lambda)$ is a translate of an idempotent. Here, $H(1)=\pi_{3}(H) \cap R^{\prime}$ is normal and $G(1) / H(1)$ is a semidirect product of $H^{\prime \prime}=\pi_{3}(H) / H(1)$ and $R^{\prime \prime}=R^{\prime} / H(1)$. 
As in Step 4 of the proof of Theorem 3.4 in [Sh3], $\pi_{3}(\lambda)=\omega_{\pi_{3}(H)} * \delta_{x}=\delta_{x} * \omega_{\pi_{3}(H)}$, where $x \in R^{\prime}$. Therefore, $N_{\lambda} \subset H L$ and since $V \subset L, G(\lambda)=\overline{V N_{\lambda}} \subset H L$. This implies that $\pi_{3}(\lambda)=\omega_{\pi_{3}(H)}=\pi_{3}(\nu)$ for any $\nu \in F_{\lambda}$. Hence, without loss of generality we may assume that $G=H L$. Since $V$ is central, $L=\overline{V R_{1}}$ has length less than $n$; hence by the induction hypothesis there exists a $\lambda$-norm $F_{\lambda}$ in $S$. This completes the proof.

\section{Proofs of Theorems 1.1, 1.2, 1.4, 1.5}

Proof of Theorem 1.1. Since $Z_{\mu}^{0} / Z^{0}$ is compact, by Theorem 2.3 there exists a closed abelian semigroup $S$ with identity $h=\omega_{H}$ in $M_{H}^{1}(G)$ such that $\mu \in S$, any $\alpha \in F_{\mu}$ is infinitesimally divisible in $S, F_{\pi(\mu)}$ is compact in $\pi(S)=S / Z^{0}$, it is idempotent-free and the set $S^{u}$ of invertible elements in $S$ is a connected abelian subgroup such that $S^{u} / Z^{0} h$ is compact. Then $S_{\xi}=S / S^{u}$ is an abelian semigroup with identity $\bar{h}=\xi(h)$, and any $\alpha \in F_{\mu}$ is infinitesimally divisible and $F_{\bar{\mu}}$ is compact, idempotent-free and associate-free in $S_{\xi}$. Also, $S$ consists of normal measures if $\Delta$ is normal.

Now suppose $\mu$ satisfies condition (1) of the hypothesis. By Proposition 6.4, for every $\lambda \in F_{\mu} \backslash S^{u}$ there exists $\alpha \in F_{\mu} \backslash S^{u}$ and a subset $F(\bar{\alpha}) \subset F_{\bar{\alpha}}$, such that $\bar{\alpha}$ and $F(\bar{\alpha})$ satisfy conditions (1)-(3) of Theorem 2.1.

Now suppose condition (2) of the hypothesis is satisfied. Let $\lambda \in F_{\mu} \backslash S^{u}$. We first show that $\lambda$ is not a translate of an idempotent. Suppose the contrary. Then since it is normal, $\lambda * \tilde{\lambda}=\tilde{\lambda} * \lambda$ is an idempotent, say $\omega_{H^{\prime}}$, for some compact group $H^{\prime}$. Then for any $\nu \in F_{\lambda}, \nu \tilde{\nu}=\tilde{\nu} \nu$ is supported on $H^{\prime}$. Also, for any two element $\alpha, \beta$ in $S, \alpha \tilde{\alpha}, \beta \tilde{\beta}$ commute. Therefore, $\lambda \tilde{\lambda}$ is infinitesimally divisible in $M_{H}^{1}\left(H^{\prime}\right)$ and hence $\lambda \tilde{\lambda}=\omega_{H}$ (cf. [Sh3], Lemma 3.1). That is, $\lambda=\delta_{y} * \omega_{H} \in S^{u}$, a contradiction.

Now by Theorem [3.8, there exists a $\lambda$-norm $f_{\lambda}$ on $F_{\lambda}$. Since $S^{u}$ is a group, $f_{\lambda}\left(S^{u}\right)=\{0\}$. Hence we can define a $\bar{\lambda}$-norm on $F_{\bar{\lambda}}$ as $f_{\bar{\lambda}}(\bar{\nu})=f_{\lambda}(\nu)$ for all $\nu \in F_{\lambda}$. Now conditions of Theorem 2.1 are satisfied by $\bar{\lambda}$ and $F_{\bar{\lambda}}$.

So in either case, by Corollary 2.2 there exists a continuous one-parameter semigroup $\{\phi(t)\}_{t>0} \subset S_{\xi}$ such that $\phi(1)=\bar{\mu}=\xi(\mu)$. In particular, for every $n$ there exist $\nu_{n} \in S$ and $a_{n} \in S^{u}$ such that $\nu_{n}^{n} a_{n}=\mu$. Since $S^{u}$ is a connected abelian group, it is divisible and hence $\mu_{n}^{n}=\mu$, for all $n$, where $\mu_{n}=\nu_{n} b_{n}$ and $b_{n}^{n}=a_{n}$. That is, $\mu$ is infinitely divisible in $F_{\mu}$ (in $S$ ) and $F_{\mu} / Z^{0}$ is compact. Now by Lemma 5.3, $A=\left\{\mu_{n}^{m} \in S \mid \mu_{n}^{n}=\mu, 1 \leq m \leq n, n \in \mathbb{N}\right\}$ is relatively compact and there exists a continuous one-parameter semigroup $\Sigma=\left\{\mu_{t}\right\}_{t \geq 0} \subset \bar{A}, \mu_{0}=\omega_{H}$, and a compact subgroup $C \subset Z_{\mu}$ which commutes with $\Sigma$, such that $\mu$ is quasiembeddable in $\Sigma$ with respect to $C$. Moreover, $\mu$ itself is embeddable if $C / H$ is arcwise connected.

Proof of Theorem 1.4. In the case $G$ is a Lie group, then every compact connected subgroup of $G$ is arcwise connected, and in the case $G$ is totally disconnected, $Z_{\mu}^{0}$ is trivial and hence so is every subgroup of it. Also condition (1) of Theorem 1.1 is satisfied and $Z_{\mu}^{0} / Z^{0}$ is compact; it implies that $\mu$ is embeddable.

In the case $G$ is $\mathbb{F}$-algebraic, then $G$ is either a Lie group or a totally disconnected group. Moreover if $G$ is real algebraic, $Z^{c}\left(G_{\mu}\right)=G$ implies that $Z_{\mu}^{0} / Z^{0}$ is compact. In the general case, if $G_{\mu}$ is amenable, Lemma 4.8 implies that $G_{\mu}$ is contained in 
a compact extension of a closed solvable normal subgroup and hence the assertion follows from above.

Proof of Theorem 1.5. If $\mu$ is the limit of a normal c.i.t.s., then condition (2) of Theorem 1.1 is satisfied and the assertion follows as in the proof of Theorem 1.4

Proof of Theorem 1.2. Let $\mu$ be the limit of any symmetric c.i.t.s. By Proposition 2.8 of [Sh3, there exists a closed subsemigroup $S$ of $M_{H}^{1}\left(G_{\mu}\right)$ with identity $\omega_{H}$ such that $F_{\mu}$ is compact, associate-free and idempotent-free; and as above, we can suppose that each $\nu \in F_{\mu}$ is infinitesimally divisible in $S$.

Now let $\lambda \in F_{\mu} \backslash\left\{\omega_{H}\right\}$. If $\lambda$ is a translate of an idempotent, then it is an idempotent, as it is symmetric, which contradicts the fact that $F_{\mu}$ is idempotentfree. Now by Theorem 3.8, there exists a $\lambda$-norm on $F_{\lambda}$ (in $S$ ), which in turn implies the embeddability of $\mu$ by Corollary 2.2 .

Remark. In the proof of Theorem[1.2 in the case of Lie projective groups, the partial homomorphisms can be obtained from Theorems 1.8 3.6 above and Corollary 3.5 of [Sh3] instead of from Theorem 3.8.

\section{Proofs of Theorems 1.61 .7}

Here, we prove a result which contains Theorems 1.6 1.7

Theorem 8.1. Let $G$ be a $\mathbb{F}$-algebraic group and let $\mu$ be the limit of c.i.t.s. such that $Z^{c}\left(G_{\mu}\right)=G$ and $\mu$ satisfies any one of the following two conditions:

(1) For some $n \in \mathbb{N}$ and some compact subgroup $H$ of $I(\mu)$, $\mu^{n}$ can be written as

$$
\mu^{n}=c \omega_{H}+\mu_{n, H},
$$

where $c \in\left[0,1\left[\right.\right.$ and $\mu_{n, H}$ is a positive measure with density and, if $\mathbb{F}$ has positive characteristic, then the radical of $G$ is $\mathbb{F}$-defined.

(2) $\mu$ has finite 1-moment.

Then $\mu$ is embeddable.

The proof will follow from the following result.

Proposition 8.2. Let $G$ be a $\mathbb{F}$-algebraic group. Let $\mu \in M^{1}(G)$ be such that $Z^{c}\left(G_{\mu}\right)=G$ and $\mu$ satisfies condition (1) or (2) of the above theorem for some $c \in[0,1[$, some $n \in \mathbb{N}$ and some $H \subset I(\mu)$. Let $S$ be a closed abelian subsemigroup of $M_{H^{\prime}}^{1}(G)$ with identity $\omega_{H^{\prime}}$ such that $\mu \in S$, every $\lambda \in F_{\mu}$ is infinitesimally divisible in $S$ and the group $S^{u}$ of invertible elements in $S$ is connected. Suppose $F_{\bar{\mu}}$ is compact, idempotent-free and associate-free. Then for every $\lambda \in F_{\mu} \backslash S^{u}$, there exist $\alpha \in F_{\lambda} \backslash S^{u}$ and a compact subset $F(\bar{\alpha}) \subset F_{\bar{\alpha}}$ in $S_{\xi}$ such that $\bar{\alpha}$ and $F(\bar{\alpha})$ satisfy conditions of Theorem 2.1 .

Proof. Without loss of generality we may assume that $F_{\mu}$ generates $S$ as a closed abelian subsemigroup of $M_{H^{\prime}}^{1}(G)$. Let $\mathbf{R}$ be the radical of $\mathbf{G}$ and let $\pi_{1}: \mathbf{G} \rightarrow \mathbf{G} / \mathbf{R}$ be the natural projection. We suppose first that the radical $\mathbf{R}$ of $\mathbf{G}$ is $\mathbb{F}$-defined and we denote by $G^{1}$ the subgroup of $\mathbb{F}$-rational points in $\mathbf{G} / \mathbf{R}$. We know that $\pi_{1}(G)$ is open and closed in $G^{1}$ (see [Ma], p. 50).

For any $\nu \in M^{1}(G)$, let $\nu^{\prime}=\pi_{1}(\nu)$. Let $\lambda \in F_{\mu} \backslash S^{u}$ and suppose $r^{\prime}=r\left(\lambda^{\prime}\right)<1$. Then $r\left(\mu^{\prime}\right)<1$, since $\lambda^{\prime} \in F_{\mu^{\prime}}$ and hence $G_{\mu^{\prime}}$ is non-amenable (cf. [DeG]). Since $G_{\mu^{\prime}} \subset \pi_{1}(G)$ and $\pi_{1}(G)$ is open and closed in $G^{1}, G^{1}$ is non-amenable, hence 
non-compact. Moreover from Theorem [3.6, it follows that $r\left(\lambda^{\prime}\right)>0$ and hence $0<r^{\prime}<1$.

Now for some $n \in \mathbb{N}$, suppose $\mu_{n, H}$ is a positive measure with density (see condition (1)), so is $\mu_{n, H}^{\prime}$ on $G^{1}$. Since $\mathbf{G} / \mathbf{R}$ is a semisimple algebraic group, $G^{1}$ is non-compact and $\mu_{n, H}^{\prime}$ has density on $G^{1}$, we can apply Theorem 4.1 and get a continuous homomorphism $f$ of $S^{\prime}=\pi_{1}(S)$ into [0,1] such that

$$
0<f\left(\mu^{\prime}\right)=r\left(\mu^{\prime}\right)<1, \quad 0<f\left(\lambda^{\prime}\right)<1 .
$$

Then we get a $\lambda$-norm on $F_{\lambda}$ by the following formula:

$$
f_{\lambda}(\nu)=-\log f\left(\nu^{\prime}\right), \quad \nu \in F_{\mu} .
$$

The condition $f\left(\mu^{\prime}\right)>0$ and the multiplicativity of $f$ implies $f\left(\nu^{\prime}\right)>0$ for any $\nu \in F_{\mu}$. Hence $f_{\lambda}$ is well defined and continuous on $F_{\lambda}$. Furthermore, since $f\left(\lambda^{\prime}\right) \leq r^{\prime}<1$, we have $f_{\lambda}(\lambda)>0$.

Now let $\mu$ satisfy the moment condition (2). Since $\pi_{1}$ is continuous as a map from $G$ to $G^{1}$, the moment condition for $\mu$ carries over to $\mu^{\prime} \in M^{1}\left(G^{1}\right)$. We also have $Z^{c}\left(G_{\mu^{\prime}}\right)=G / R$. Hence we can apply Lemma 4.10. Since $G_{\lambda^{\prime}}$ is non-amenable, there exists $s \in \hat{\mathcal{A}}^{+}$, such that $s\left[L\left(\lambda^{\prime}\right)\right]>0$. Then we get a $\lambda$-norm through the formula

$$
f_{\lambda}(\nu)=s\left[L\left(\nu^{\prime}\right)\right], \quad \nu \in F_{\mu} .
$$

Clearly, $f_{\lambda}$ is a continuous function on $F_{\mu}$ and $f_{\lambda}(\lambda)=s\left[L\left(\lambda^{\prime}\right)\right]>0$ (cf. Theorem (1.9).

In both of the above cases, since $f_{\lambda}\left(S^{u}\right)=\{0\}$, we can define a $\bar{\lambda}$-norm on $F_{\bar{\lambda}}$ as $f_{\bar{\lambda}}=f_{\lambda} \circ \xi^{-1}$, which is well defined, and the conditions of Theorem 2.1 are satisfied for $\alpha=\lambda$ and $F(\bar{\alpha})=F_{\bar{\lambda}}$.

Now suppose $r\left(\lambda^{\prime}\right)$ is 1 . Then $G_{\lambda^{\prime}}$ is amenable, so is $\pi_{1}^{-1}\left(G_{\lambda^{\prime}}\right)$, as well as its closed subgroup $G_{\lambda}$. Now, since we want to have $F_{\lambda}$ to satisfy conditions of Theorem 2.1 without loss of generality we may take $S(1)$ to be the closed semigroup generated by $F_{\lambda}$ and show that it satisfies the conditions of Theorem 2.1. Let $G(\lambda)$ be the closed subgroup of $G$ generated by $\left\{\operatorname{supp} \nu \mid \nu \in F_{\lambda}\right\}$. Since the elements of $\lambda$ are supported on cosets of $G_{\lambda}$ in $N\left(G_{\lambda}\right)$, the normaliser of $G_{\lambda}$ in $G$, and since $S$ is abelian, we have that $G(\lambda) / G_{\lambda}$ is abelian and hence $G(\lambda)$ is amenable. Hence from Lemma 4.8, $G(\lambda)$ is contained in a compact extension of a closed solvable normal subgroup. Here, $S(1) \subset M^{1}(G(\lambda)), S^{u} \subset S(1)$, and $S(1)_{\xi}=\xi[S(1)]$. Now by Proposition [6.4, there exist $\alpha \in F_{\lambda}$ and a compact subset $F(\bar{\alpha}) \subset F_{\bar{\alpha}}$ such that $\bar{\alpha}$ and $F(\bar{\alpha})$ satisfy conditions of Theorem [2.1.

In the case $\mathbf{R}$ is not $\mathbb{F}$-defined and $\mu \in M^{1, m}(G)$, we consider a finite algebraic extension $\mathbb{F}^{\prime}$ of $\mathbb{F}$ such that $\mathbf{R}$ is $\mathbb{F}^{\prime}$-defined and we replace $G$ by $G^{\prime}$, the subgroup of $\mathbb{F}^{\prime}$-rational points of $\mathbf{G}$. The Zariski closure condition of $G_{\mu}$ is also satisfied inside $G^{\prime}$, and moreover since $G$ is closed in $G^{\prime}$, we have also $\mu \in M^{1, m}\left(G^{\prime}\right)$. Hence we are reduced to the above situation and the assertion follows.

Proof of Theorem 8.1. Let $\mathbf{G}$ be a $\mathbb{F}$-algebraic group. Let $\mu \in M^{1}(G)$ be the limit of a c.i.t.s. such that $Z^{c}\left(G_{\mu}\right)=G$. Suppose $G$ is real algebraic. Now since the centraliser of $G_{\mu}, Z\left(G_{\mu}\right)=Z$, by Theorem 3.2 of [DM], $F_{\mu} / Z$, and hence $F_{\mu} / Z^{0}$ is compact as $Z$ has only finitely many connected components. In particular, $Z_{\mu}^{0} / Z^{0} \subset$ $F_{\mu} / Z^{0}$ is also compact. If $\mathbb{F}$ is ultrametric, then $G$ is totally disconnected and $Z_{\mu}^{0}=\{e\}=Z^{0}$. Now by Theorem 2.3 , there exists a closed abelian semigroup $S \subset$ $M_{H^{\prime}}^{1}(G)$ with identity $\omega_{H^{\prime}}$ such that $\mu \in S$, every element in $F_{\mu}$ is infinitesimally 
divisible and $F_{\pi(\mu)}$ is compact and idempotent-free, and the group $S^{u}$ of invertible elements in $S$ is connected. This implies that $S_{\xi}=S / S^{u}$ is an abelian semigroup with identity $\xi\left(\omega_{H^{\prime}}\right), F_{\xi(\mu)}$ is compact, idempotent-free and associate-free and every element in $F_{\xi(\mu)}$ is infinitesimally divisible.

Now, since $\mu$ satisfies condition (1) or (2) of the hypothesis, by Proposition 8.2 the conditions of Theorem 2.1 are satisfied. Therefore, we get as in the last step of the proof of Theorem 1.1 that $\mu$ is embeddable.

Remark. It is obvious from the proof of Theorem 8.1 that, on a Lie group $G$ with finitely many connected components, a measure $\mu$ is embeddable if $\mu$ is the limit of a c.i.t.s., $Z_{\mu}^{0} / Z^{0}$ is compact and $\mu$ satisfies condition (1) of the theorem.

\section{Complements}

The condition that $Z_{\mu}^{0} / Z^{0}$ is compact is a fullness condition on $\mu$ which is used in particular in Theorem 2.3 in order to get infinitesimal divisibility of $\mu$. A seemingly more natural condition is the compactness of $Z_{\mu}^{0} /\left[Z\left(G_{\mu}\right)\right]^{0}$, since it is satisfied by Dirac measures as well as any measure $\mu$ on an algebraic group (cf. Theorem 3.2, [DM] ) (where $Z\left(G_{\mu}\right)$ is the centraliser of $G_{\mu}$ in $G$ ). We discuss below the relations of these two conditions with embeddability, infinitesimal divisibility and being the limit of a c.i.t.s., starting from the case of Dirac measures. The first example shows that the compactness of $Z_{\mu}^{0} / Z^{0}$ is necessary but the compactness of $Z_{\mu}^{0} /\left[Z\left(G_{\mu}\right)\right]^{0}$ is not sufficient.

Let $G$ be a connected Lie group, with Lie algebra $\mathcal{G}$, such that the image of the exponential map is not closed. Let $\mu_{n}=\delta_{g_{n}}=\exp X_{n}$ be a sequence of Dirac measures with $X_{n} \in \mathcal{G}$ and $\mu=\delta_{g}=\lim _{n} \mu_{n}$ with $g \notin \exp \mathcal{G}$. Since we have $g_{n}=\left(\exp \frac{X_{n}}{n}\right)^{n}$, it is clear that $\mu_{n}$ defines a c.i.t.s. converging to $\mu$. However, $\mu=\delta_{g}$ cannot be embedded in a one-parameter (semi)group, contrary to the conclusion of Theorems [1.4, 1.5 and 1.3. We observe from Theorem 1.5 that $Z_{\mu}^{0} / Z^{0}$ cannot be compact in such a situation.

A corresponding simple example is the following:

Example 1. Let $G=S L(2, \mathbb{R})$. Its Lie algebra $\mathcal{G}$ is given by $\mathcal{G}=\{X \in M(2, \mathbb{R}) \mid$ $\operatorname{Tr} X=0\}$. It can be easily verified that

$$
G \backslash \exp \mathcal{G}=\{g \in G \mid g \neq-I d, \operatorname{Tr} g \leq-2\} .
$$

Hence the boundary of $\exp \mathcal{G}$ is

$$
\overline{\exp \mathcal{G}} \backslash \exp \mathcal{G}=\{g \in G \mid g \neq-I d, \operatorname{Tr} g=-2\} .
$$

It is non-trivial and consists of the matrices $g \neq-\mathrm{Id}$, with the unique eigenvalue -1 .

Theorem 9.1. Let $G$ be any locally compact group and $\mu=\delta_{x}$, for some $x \in G$. Then the following are equivalent:

(a) $\mu=\delta_{x}$ is infinitesimally divisible in $M^{1}(G)$.

(b) $x$ belongs to a connected abelian subgroup.

Moreover, if $G$ is a Lie group then (b) can be replaced by

$\left(\mathrm{b}^{\prime}\right) x$ belongs to a one-parameter subgroup. 
Proof. (b) implies (a) since, if we denote by $G_{x}$ the closure of the connected abelian subgroup containing $x$, then $G_{x}=\bigcup_{n=1}^{\infty} V^{n}$ for any arbitrarily small neighbourhood $V$ of $e$ in $G_{x}$. Conversely, if $\mu=\delta_{x}$ is infinitesimally divisible, it is infinitesimally divisible in the centraliser of $\mu, Z\left(G_{\mu}\right)=Z(x)$, the centraliser of $x$. Also, applying Lemma 3.4 of [Sh4] to the projection of $\mu=\delta_{x}$ on $Z(x) / Z^{0}(x)$, where $Z^{0}(x)$ is the connected component of $Z(x)$, we get that $x \in Z^{0}(x)$. That is, $x$ is in the center of $Z^{0}(x)$. Since the connected group $Z^{0}(x)$ is Lie projective, it is enough to show that any element in the center of a connected Lie group is contained in an abelian analytic subgroup, which is connected and this follows from Theorem 1.2 in Ch. XVI of $[\mathrm{Ho}$.

In view of Theorem 9.1 one can ask if the compactness of $Z_{\mu}^{0} /\left[Z\left(G_{\mu}\right)\right]^{0}$ and infinitesimal divisibility of $\mu$ are sufficient to ensure the embeddability of $\mu$. This is indeed the case if $G$ is connected nilpotent, a fact which improves Theorem 4.1 in Sh4 (see the proof). Also, in this direction we have the following theorem which can easily be deduced from the proof of Theorem 4.1 in Sh4 using Theorem 3.8.

Theorem 9.2. Let $G$ be a locally compact group and let $\mu \in M^{1}(G)$ be such that $Z_{\mu}^{0} /\left[Z\left(G_{\mu}\right)\right]^{0}$ is compact. Suppose $\mu$ is infinitesimally divisible into normal measures, i.e. for every neighbourhood $U$ of $\delta_{e}$, there exist normal measures $\lambda_{i} \in$ $U, i=1, \ldots, n$, such that $\lambda_{i}$ 's commute and $\mu=\lambda_{1} * \cdots * \lambda_{n}$. Then $\mu$ is weakly infinitely divisible, i.e. for every $n \in \mathbb{N}$, there exist a normal measure $\mu_{n} \in M^{1}(G)$ and $x_{n} \in G$ such that $\mu=\mu_{n}^{n} x_{n}$ and $x_{n} \mu=\mu x_{n}$.

In order to show that the condition of finiteness of 1-moment can be satisfied for one-parameter semigroups, we consider the following simple family of oneparameter semigroups on a group $G$ :

Example 2. Let $S_{r, \nu}=\left\{\mu_{t}\right\}_{t \geq 0}$, where $r \in\left[0,1\left[\right.\right.$, and let $\nu \in M^{1}(G)$ be defined as follows:

$$
\begin{gathered}
\mu_{t}=(1-r)^{t}\left[\delta_{e}-r \nu\right]^{-t}, \\
\mu_{t}=(1-r)^{t}\left[\delta_{e}+\sum_{k=1}^{\infty} \frac{t(t+1)(t+k-1)}{k !} r^{k} \nu^{k}\right] .
\end{gathered}
$$$$
\text { i.e. }
$$

Then from (1), $S_{r, \nu}=\left\{\mu_{t}\right\}_{t \geq 0}$ is a one-parameter semigroup of $M^{1}(G)$. Since $\nu^{k}\left(\delta_{V}\right) \leq k \nu\left(\delta_{V}\right)$, we see that $\mu=\mu_{1}$ has a finite 1-moment if and only if $\nu$ has.

Now we give an example showing that condition (1) of Theorem 8.1 can be satisfied for a group $G$ with finitely many connected components.

Example 3. Suppose $H$ is a compact subgroup of $G, f \in L_{+}^{1}(G)$ is $H$-bi-invariant and $\int f(g) \mathrm{d} g=1$. Let $\nu=f(g) \mathrm{d} g$ and consider the one-parameter semigroup in $M^{1}(G): \mu_{t}=\exp t\left(\nu-\omega_{H}\right)$. We have

$$
\mu_{1}=\frac{1}{e}\left[\omega_{H}+\sum \frac{\nu^{k}}{k !}\right] .
$$

Here, $\mu_{1}=\mu$ satisfies condition (1) of Theorem 8.1 for $n=1$ and $c=1 / e$. 


\section{REFERENCES}

[Be] Y. Benoist. Propriétés asymptotiques des groupes linéaires. Geom. Func. Anal. 7 (1997), 1-47. MR 98b:22010

[BC] C. Berg and J.P.R. Christensen. Sur la norme des opérateurs de convolution. Invent. Math. 23 (1974), 173-176. MR 49:3449

[Bo] A. Borel. Linear algebraic groups, 2nd enlarged edition. Springer (1991). MR92d:20001

[Ca] H. Carnal. Deux théorèmes sur les groupes stochastiques compacts. Comm. Math. Helv. 40 (1966), 237-246. MR33:8009

[DM] S. G. Dani and M. McCrudden. Embeddability of infinitely divisible distributions on linear Lie groups. Invent. Math. 110 (1992), 237-261. MF94d:60010

[DSh] S. G. Dani and Riddhi Shah. Collapsible measures and concentration functions on Lie groups, Math. Proc. Camb. Phil. Soc. 122 (1997), 105-113. MR.98e:60015

[DeG] Y. Derriennic and Y. Guivarc'h. Théorème de renouvellement pour les groupes non moyennables. C. R. A. S., Paris 277 (1973), 613-616. MR48:7332

[Fe] P. Feinsilver. Processes with independent increments on a Lie group. Trans. Amer. Math. Soc. 242 (1978), 73-121. MR.58:3063

[F] W. Feller. An Introduction to probability theory and its applications Vol. II. Wiley (1966). MR $35: 1048$

[Fu] H. Furstenberg. Boundary theory and stochastic processes on homogeneous spaces. Proc. Symp. Pure Math. 36 (1972), 193-229. MF50:4815

[Ga] R. Gangolli. Isotropic infinitely divisible measures on symmetric spaces. Acta Math. 111 (1964), 213-246. MR28:4557

[GoMa] I. Ya. Goldsheid and G. A. Margulis. Lyapunov exponents of a product of random matrices. Russian Math. Surveys 44 (1989), 11-71. MF91j:60014

[Gr] U. Grenander. Probabilities on algebraic structures. Almquist and Wiksell. StockholmGöteberg-Uppsala (1963).

[G1] Y. Guivarc'h. Croissance polynomiale et périodes des fonctions harmoniques. Bull. S.M.F. 101 (1973), 333-379. MR51:5841

[G2] Y. Guivarc'h. Sur la loi des grands nombres et le rayon spectral d'une marche aléatoire. Astérisque 74 (1980), 47-98. MF 82g:60016

[G3] Y. Guivarc'h. Produits de matrices aléatoires et applications aux propriétés géométriques des sous-groupes du groupe linéaire. Ergod. Th. Dyn. Syst. 10 (1990), 483-512. MR.92c:60011

[G4] Y. Guivarc'h. Limit theorems for random walks and products of random matrices. To appear in: Proceedings of the CIMPA-TIFR School on Probability Measures on Groups, Mumbai 2002. TIFR Studies in Mathematics series.

[GR1] Y. Guivarc'h and A. Raugi. Frontière de Furstenberg, propriétés de contraction et théorèmes de convergence. Z. Wahr. Verw. Gebiete 69 (1985), 187-242. Mr86h:60126

[GR2] Y. Guivarc'h and A. Raugi. Products of random matrices and convergence theorems. In: Random Matrices (Kesten Cohen-Newmann Eds.) Contemporary Math. AMS 50 (1986), 31-54. MF $87 \mathrm{~m}: 60024$

$[\mathrm{H}] \quad$ H. Heyer. Probability measures on locally compact groups, Springer-Verlag, BerlinHeidelberg (1977). MR.58:18648

[Ho] G. P. Hochschild. The structure of Lie groups. Holden-Day (1965). MR.34:7696

$[\mathrm{Hu}]$ G. A. Hunt. Semigroups of measures on Lie groups. Trans. Amer. Math. Soc. 81 (1956), 264-293. MR 18:54a

[JRoW] W. Jaworski, J. Rosenblatt and G. Willis, Concentration functions in locally compact groups. Math. Ann. 305 (1996), 673-691. MR97k:43001

[Ke] D. G. Kendall. Delphic semigroups, infinitely divisible regenerative phenomena and the arithmetic of $p$-functions. Z. Wahr. Verw. Gebiete 9 (1968), 163-195. MR37:5320

[KRut] M. G. Krĕn and M. A. Rutman. Linear operators leaving invariant a cone in a Banach space. A.M.S. Transl. 26 (1950), 200-325. MR 12:341b

[L] P. Lévy. Processus stochastiques et mouvement brownien. Gauthier-Villars, (1948). MR.10:551a

[Ma] G. A. Margulis. Discrete subgroups of semisimple Lie groups. Ergebnisse der Math., Springer-Verlag (1991). MR92h:22021 
[M] M. McCrudden. Factors and roots of large measures on connected Lie groups, Math. Zeits. 177 (1981), 315-322. MR82m:60014

[P] K. R. Parthasarathy. Probability measures on metric spaces. Academic Press, New YorkLondon (1967). MR 37:2271

[PRaV] K. R. Parthasarathy, R. Ranga Rao and S. R. S. Varadhan. Probability distributions on locally compact abelian groups, Illinois J. Math. 7 (1963), 337-369. MR.32:8378

[Pr] G. Prasad. $\mathbb{R}-$ regular elements in Zariski-dense subgroups. Quarterly J. Math. 45 (1994), 541-545. MR 96a:22022

[R] M. S. Raghunathan. A proof of Oseledec's multiplicative ergodic theorem. Israel J. Math. 32 (1979), 356-362. MR81f:60016

[Ruz] I. Z. Ruzsa. Infinite divisibility. Adv. Math. 69 (1988), 115-132. MR.89g:60051

[RuzS] I. Z. Ruzsa and G. J. Szekely. Algebraic probability theory. Wiley (1988). MR89j:60006

[Sh1] Riddhi Shah. Semistable measures and limit theorems on real and $p$-adic groups. Mh. Math. 115 (1993), 191-213. MR95g:60013

[Sh2] Riddhi Shah. Limits of commutative triangular systems on real and $p$-adic groups. Math. Proc. of Camb. Phil. Soc 120 (1996), 181-192. MR97e:60015

[Sh3] Riddhi Shah. The central limit problem on locally compact group. Israel J. Math. 110 (1999), 189-218. MR2000m:60007

[Sh4] Riddhi Shah. Limits of commutative triangular systems on locally compact groups. Indian Academy of Sciences Proceeding (Math. Sci.) 111 (2001), 49-63. MR.2002b:60003

[Si] E. Siebert. Fourier analysis and limit theorems for convolution semigroups on a locally compact group. Adv. Math. 39 (1981), 111-154. MR82h:60023

[StV] D. W. Stroock and S. R. S. Varadhan. Limit theorems for random walks on Lie groups. Sankhyā Ser. A 35 (1973), no. 3, 277-294. MR58:24457

[T1] J. Tits. Reductive groups over local fields. Proc. Sympos. Pure Math. 33 (1977), 29-69. MR 80h:20064

[T2] J. Tits. Représentations linéaires irréductibles d'un groupe réductif sur un corps quelconque. J. Reine Angew. Math. 247 (1971), 196-220. MR 43:3269

[W] D. F. Wehn. Probabilities on Lie groups. Proc. Nat. Acad. Sci. USA 48 (1962), 791-795. $\operatorname{MR} 27: 3011$

IRMaR, Université de Rennes1, Campus de Beaulieu, 35042, Rennes Cedex, France

E-mail address: yves.guivarch@univ-rennes1.fr

School of Mathematics, Tata Institute of Fundamental Research, Homi Bhabha Road, Mumbai 400 005, India

E-mail address: riddhi@math.tifr.res.in 